\title{
SUPERRIGIDITY OF ACTIONS ON FINITE RANK MEDIAN SPACES
}

\author{
ELIA FIORAVANTI
}

\begin{abstract}
Finite rank median spaces are a simultaneous generalisation of finite dimensional $\mathrm{CAT}(0)$ cube complexes and real trees. If $\Gamma$ is an irreducible lattice in a product of rank-one simple Lie groups, we show that every action of $\Gamma$ on a complete, finite rank median space has a global fixed point. This is in sharp contrast with the behaviour of actions on infinite rank median spaces, where even proper cocompact actions can arise.

The fixed point property is obtained as corollary to a superrigidity result; the latter holds for irreducible lattices in arbitrary products of compactly generated topological groups.

We exploit Roller compactifications of median spaces; these were introduced in [Fio17] and generalise a well-known construction in the case of cube complexes. We show that the Haagerup cocycle provides a reduced 1-cohomology class that detects group actions with a finite orbit in the Roller compactification. This is new even for CAT(0) cube complexes and has interesting consequences involving Shalom's property $H_{F D}$. For instance, in Gromov's density model, random groups at low density do not have $H_{F D}$.
\end{abstract}

\section{COnTEnts}

1. Introduction.

2. Preliminaries. 10

2.1. Median spaces and median algebras.

2.2. Bridges. 21

2.3. The Haagerup class. 23

3. Haagerup class and elementarity of actions. 24

3.1. The main statement. 24

3.2. Elementarity and Shalom's property $H_{F D}$. 27

4. Superrigidity. 28

4.1. The superrigidity result. 28

4.2. Homomorphisms to coarse median groups. 35

Appendix A. Structure of UBS's. 36

References 42 


\section{INTRODUCTION.}

A median space is a metric space $(X, d)$ such that, for any three points $x_{1}, x_{2}, x_{3} \in X$, there exists a unique point $m=m\left(x_{1}, x_{2}, x_{3}\right) \in X$ with the property that $d\left(x_{i}, m\right)+d\left(m, x_{j}\right)=d\left(x_{i}, x_{j}\right)$ for all $1 \leq i<j \leq 3$. Simple examples are provided by real trees and by $\mathbb{R}^{n}$ with the $\ell^{1}$ metric.

We remark that, to each connected median space $X$ of finite topological dimension, there corresponds a canonical CAT $(0)$ space $\widehat{X}$, which is bi-Lipschitz equivalent to $X$ Bow16. For instance, to $\mathbb{R}^{n}$ with the $\ell^{1}$ metric we associate $\mathbb{R}^{n}$ with its euclidean distance.

More elaborate examples of median spaces are provided by Guirardel cores Gui05 and by simply connected cube complexes satisfying Gromov's link condition [Gro87]. In the latter case, we obtain a median space $X$ by endowing each cube with the $\ell^{1}$ metric, while $\widehat{X}$ is the corresponding CAT(0) cube complex. Median spaces generally display wilder features than cube complexes, as, like real trees, they can be essentially non-discrete objects.

A clear illustration of this is the fact that, unlike cube complexes, the class of median spaces is closed under ultralimits. In particular, any asymptotic cone of any hierarchically hyperbolic group [BHS17a] is described by a median space [BDS11a, BDS11b, Bow13a, Zei16]. Furthermore, we have observed in Fio18 that general group actions on median spaces need not yield codimension-one subgroups; this is in contrast with a well-known key property of cube complexes [Sag95, Ger97].

These phenomena already appear among (connected) median spaces of finite rank, i.e. those median spaces where the topological dimensions of locally compact subsets are uniformly bounded. Finite rank median spaces will be the main focus of the present paper and, indeed, all the examples listed above have finite rank.

Finite rank median spaces also retain many of the good combinatorial properties of cube complexes. In addition to the existence of a CAT(0) metric, their horoboundaries are compatible with the median property [Fio17] and many groups of isometries contain free non-abelian subgroups [Fio18]. We would expect many known results for $\mathrm{CAT}(0)$ cube complexes to extend to finite rank median spaces without significant complications, for instance [BCG ${ }^{+} 09$, GH10, NS13, CFI16, KS16, Fer18, FLM18] to name a few.

All the above similarities between cube complexes and more general median spaces can be ascribed to the existence of a collection $\mathscr{W}$ of walls. These encode the geometry of the space in the same way as hyperplanes do in CAT(0) cube complexes. The set $\mathscr{W}$ should not be thought of as discrete and, in fact, it needs to be endowed with a measure $\mu$ that expresses the "thickness" of sets of walls [CDH10, Fio17]. Indeed, the concept of median space is dual to the notion of space with measured walls [CMV04, dCTV08, CDH10] - similar to spaces with walls [HP98 being the dual viewpoint on CAT(0) cube complexes [Sag95, Nic04, CN05]. 
Our main theorem is a superrigidity result for irreducible lattices $\Gamma$ in products $G=G_{1} \times \ldots \times G_{\ell}$ of locally compact, compactly generated groups. Namely, under weak assumptions of non-elementarity, every action of $\Gamma$ on a finite rank median space $X$ essentially arises from continuous isometric actions $G_{i} \curvearrowright Y_{i}$ on median spaces of lower rank. For cube complexes, this was known due to CFI16.

In the more general context of $\mathrm{CAT}(0)$ spaces, similar results were obtained long ago in [Mon06, CM09. Unfortunately, applying these to a median space $X$ only provides actions of the factors $G_{i}$ on CAT $(0)$ subspaces $Z_{i} \subseteq \widehat{X}$; these subspaces might bear no relation to the median structure on $X$. This might seem like an irrelevant subtlety, but it is, on the contrary, key to the fixed point properties that this paper provides.

As an illustration of this, consider a uniform irreducible lattice $\Gamma$ in the product $S L_{2}(\mathbb{R}) \times S L_{2}(\mathbb{R})$. It acts properly and cocompactly on the CAT(0) space $\mathbb{H}^{2} \times \mathbb{H}^{2}$. In particular, it is quasi-isometric to a product of surface groups, hence coarse median of finite rank (in the sense of [Bow13a]). It was moreover shown in CD17] that $\Gamma$ acts properly and cocompactly on a median space of infinite rank. It should thus appear particularly striking that every action of $\Gamma$ on a complete, connected, finite rank median space fixes a point; this follows from our results, see Corollary D below.

The proof of our superrigidity theorem follows a very similar outline to Monod's [Mon06]. This is mostly hidden in our application of Shalom's superrigidity (Theorem 4.1 in [Sha00]), thus we believe it is important to highlight the analogy here. We have already mentioned that, to each finite rank median space $X$, one can associate a finite dimensional CAT(0) space $\widehat{X}$. However, one can also consider the infinite dimensional CAT(0) (in fact, Hilbert) space $L^{2}(\mathscr{W}, \mu)$.

Retracing the proof of Monod's superrigidity in our context, we would start with an action $\Gamma \curvearrowright X$ and induce a continuous action of $G$ on the space $L^{2}(G / \Gamma, \widehat{X})$ (see Mon06 for a definition). Then, we would prove that a subspace of $L^{2}(G / \Gamma, \widehat{X})$ splits as a product $Z_{1} \times \ldots \times Z_{k}$, where the action of $G$ on the $i$-th factor only depends on the projection to $G_{i}$. Finally, we would carry back to $\Gamma \curvearrowright \widehat{X}$ the information gained about $\Gamma \curvearrowright L^{2}(G / \Gamma, \widehat{X})$.

Our application of Shalom's machinery instead constructs a continuous action of $G$ on the space $L^{2}\left(G / \Gamma, L^{2}(\mathscr{W}, \mu)\right)$. Again, one then proves a splitting theorem for this action and carries the gained insight back to the action $\Gamma \curvearrowright L^{2}(\mathscr{W}, \mu)$. Our main contribution lies in transferring information back and forth between the actions $\Gamma \curvearrowright L^{2}(\mathscr{W}, \mu)$ and $\Gamma \curvearrowright X$. In particular, Shalom's machinery can only be set in motion once we have a nonvanishing reduced cohomology class for $\Gamma \curvearrowright L^{2}(\mathscr{W}, \mu)$. We provide this by a careful study of the Haagerup cocycle, which is entirely new even in the context of $\mathrm{CAT}(0)$ cube complexes and has many interesting consequences regarding Shalom's property $H_{F D}$ (see Theorem $\mathrm{G}$ and Corollaries $\mathrm{H}$ and $\mathrm{I}$ below).

We now describe our results in greater detail. 
1.1. A cohomological characterisation of elementary actions. Each median space $X$ has a distinguished collection $\mathscr{H}$ of subsets called halfspaces; every wall gives rise to two halfspaces and each halfspace arises from a wall. The collection $\mathscr{H}$ is equipped with a measure $\widehat{\nu}$, see [Fio17. In the case of cube complexes, one recovers the usual notion of halfspace and $\widehat{\nu}$ is simply the counting measure.

Given a topological group $G$ and an isometric action $G \curvearrowright X$, one naturally obtains a unitary representation $\rho: G \rightarrow \mathscr{U}\left(L^{2}(\mathscr{H}, \widehat{\nu})\right)$ and a cocycle $b: G \rightarrow L^{2}(\mathscr{H}, \widehat{\nu})$ - the Haagerup cocycle. This construction is well-known and appears for instance in [CMV04, dCTV08, CDH10, FV17]. If $G \curvearrowright X$ has continuous orbits, $\rho$ and $b$ are continuous; thus $b$ induces a reduced continuous cohomology class $\overline{[b]} \in \overline{H_{c}^{1}}(G, \rho)$.

In [Fio18], we introduced a notion of elementarity for actions on median spaces; namely, we say that $G \curvearrowright X$ is Roller elementary if $G$ has at least one finite orbit within the Busemann compactification $\bar{X}$; the latter is also known as Roller compactification. Roller elementarity implies - but is in general strictly stronger than - the existence of a finite orbit in the visual compactification of the CAT(0) space $\widehat{X}$.

If $G \curvearrowright X$ is an isometric action with continuous orbits, Roller elementarity can be described in terms of the Haagerup class $\overline{[b]}$.

Theorem A. Let $X$ be a complete, finite rank median space. The Haagerup class $\overline{[b]} \in \overline{H_{c}^{1}}(G, \rho)$ vanishes if and only if $G \curvearrowright X$ is Roller elementary.

Theorem A extends various known results. In the case of simplicial trees, it appears in [FV17]. For CAT(0) cube complexes, the implication "Roller nonelementary $\Rightarrow \overline{[b]} \neq 0$ " is implicit in [DP16]. In [CFI16], the authors construct a family of bounded cohomology classes detecting Roller elementarity in $\mathrm{CAT}(0)$ cube complexes.

We remark that Theorem A equally holds if we replace $L^{2}(\mathscr{H}, \widehat{\nu})$ with any $L^{p}(\mathscr{H}, \widehat{\nu}), 1 \leq p<+\infty$, although it is slightly simpler to exploit the richer structure of Hilbert spaces in its proof.

Our superrigidity result only relies on the implication of Theorem A that yields $\overline{[b]} \neq 0$, but we believe the full statement of Theorem A to be of independent interest. The proof of the other implication turns out to be quite technical and requires a careful study of the structure of unidirectional boundary sets (UBS's) in median spaces; these are a generalisation of the simplices in Hagen's simplicial boundary of a CAT(0) cube complex Hag13. Most of these details will be relegated to the appendix.

1.2. Superrigidity of actions. Once we have a nontrivial reduced cohomology class, as provided by Theorem A, we can apply well-established machinery (namely Theorem 4.1 in [Sha00]) to obtain superrigidity results.

Let $X$ be a complete, finite rank median space. Its Roller compactification $\bar{X}$ is partitioned into components [Fio17. The subset $X \subseteq \bar{X}$ forms a whole component and every other component is itself a complete median space of 
strictly lower rank. This aspect of $\bar{X}$ shares many similarities with refined boundaries of CAT(0) spaces [Lee00, Cap09] and with Satake compactifications of symmetric spaces [BJ06, BJ07].

Given a component $Z \subseteq \bar{X}$, a median subalgebra of $Z$ is a subset $Y \subseteq Z$ that is itself a median space with the restriction of the median metric of $Z$; equivalently, the median map $m: Z^{3} \rightarrow Z$ takes $Y^{3}$ into $Y$. We are now ready to state our main superrigidity result.

Theorem B. Let $X$ be a complete, finite rank median space. Let $\Gamma$ be a uniform, irreducible lattice in a product $G=G_{1} \times \ldots \times G_{\ell}$ of compactly generated, locally compact groups with $\ell \geq 2$. Suppose $\Gamma \curvearrowright X$ is a Roller nonelementary action. There exist a finite index subgroup $\Gamma_{0} \leq \Gamma, a \Gamma_{0}$ invariant component $Z \subseteq \bar{X}$ and a $\Gamma_{0}$-invariant closed median subalgebra $Y \subseteq Z$ where the action $\Gamma_{0} \curvearrowright Y$ extends to a continuous action $G_{0} \curvearrowright Y$, for some open finite index subgroup $G_{0} \leq G$.

Remarks. (1) Theorem B also applies to nonuniform lattices, as long as they are square-integrable; this is a well-known technical condition that implies finite generation and ensures that Theorem 4.1 in [Sha00] still holds.

All irreducible lattices in $G_{1} \times \ldots \times G_{\ell}$ are square-integrable if each $G_{i}$ is the group of $k_{i}$-rational points of a semisimple, almost $k_{i}$-simple, $k_{i}$-isotropic linear algebraic group defined over some local field $k_{i}$ [Sha00]. Further examples of nonuniform square-integrable lattices include minimal Kac-Moody groups over sufficiently large finite ground fields; these can be regarded as irreducible lattices in the product of the closed automorphism groups of the associated buildings Rém99, Rém05].

(2) Theorem B should be compared to Shalom's superrigidity result for actions on simplicial trees, Theorem 0.7 in Sha00. If $X$ is a simplicial tree, $Y$ is always a subcomplex of $X$ and $\Gamma_{0}=\Gamma, G_{0}=G$. The complications in the statement of Theorem $B$ reflect phenomena that do not happen in the world of trees.

However, as soon as we leave the context of rank-one median spaces (i.e. real trees), our result is optimal even if one restricts to $\operatorname{CAT}(0)$ square complexes; see Examples 4.7 and 4.8. We remark that, when $X$ is a general CAT(0) square complex, the median algebra $Y$ might not be a subcomplex of $X$ or $Z$.

(3) We can take $\Gamma_{0}=\Gamma, G_{0}=G$ and $Z=X$ as long as $X$ is irreducible, $G$ has no finite orbits in the visual compactification of $\widehat{X}$ and $G$ leaves invariant no proper closed convex subset of $X$; see Theorem 4.4 below. However, even in this case, the action in general extends only to a proper median subalgebra of $X$.

(4) For CAT(0) cube complexes, the superrigidity result of [CFI16 is slightly more general than Theorem $\mathrm{B}$ as it applies to all nonuniform lattices. This is due to their use of bounded cohomology (namely 
Theorem 16 in BM02]), rather than reduced cohomology. In the setting of CAT(0) cube complexes, our strategy of proof was instead hinted at on page 9 of [Sha00.

1.3. Fixed point properties for lattices. Unlike automorphism groups of $\operatorname{CAT}(0)$ cube complexes, the isometry group of a median space needs not be totally disconnected. Still, it is possible to exploit Theorem B to derive a fixed point property for irreducible lattices in connected groups.

Given a locally compact topological group $Q$, we denote the connected component of the identity by $Q^{0}$. We say that $Q$ satisfies condition (*) if $Q / Q^{0}$ is amenable or has Shalom's property $H_{F D}$ (see Section 1.5 for a definition). In particular, all almost-connected and connected-by-(T) groups satisfy condition $(*)$.

Theorem C. Let $X$ be a complete, finite rank median space. Let $\Gamma$ be a square-integrable, irreducible lattice in a product $G_{1} \times \ldots \times G_{\ell}$ with $\ell \geq 2$. Suppose that every $G_{i}$ is compactly generated and satisfies condition (*).

(1) Every action $\Gamma \curvearrowright X$ is Roller elementary.

(2) If $\Gamma$ does not virtually map onto $\mathbb{Z}$, every action $\Gamma \curvearrowright X$ has a finite orbit within $X$. If moreover $X$ is connected, every action $\Gamma \curvearrowright X$ has a global fixed point.

When $X$ is a real tree, Theorem Calso follows from Theorem 6 in [Mon06]. We remark that, contrary to part 2 of Theorem C, every group that virtually maps onto $\mathbb{Z}$ admits a Roller elementary action on $\mathbb{R}^{n}$ with unbounded orbits.

Corollary D. Let $X$ be a complete, connected, finite rank median space. Let $\Gamma$ be any irreducible lattice in a connected, higher rank, semisimple Lie group $G$. Every action $\Gamma \curvearrowright X$ fixes a point.

The assumption that $X$ have finite rank is essential for Corollary $\mathrm{D}$ to hold. If at least one simple factor $G_{i}<G$ is locally isomorphic to $O(n, 1)$ or $U(n, 1), n \geq 2$, the lattice $\Gamma$ admits an action on an infinite rank median space with unbounded orbits [CDH10]. On the other hand, if each simple factor of $G$ has rank at least two, then $\Gamma$ has property $(\mathrm{T})$ and Corollary $\mathrm{D}$ follows from Theorem 1.2 in $\mathrm{CDH} 10$.

An analogue of Corollary $\mathrm{D}$ for CAT(0) cube complexes was proved in CFI16. We remark however that the full strength of Corollary D reveals a surprising new phenomenon. If all $G_{i}$ 's are locally isomorphic to $O(n, 1)$ and $\Gamma$ is uniform, it was shown in CD17 that $\Gamma$ admits a proper and cocompact action on an infinite rank median space. Corollary $D$ shows such actions cannot be reduced to actions on finite rank median spaces. By contrast, any cocompact $\mathrm{CAT}(0)$ cube complex is finite dimensional.

1.4. Homomorphisms to coarse median groups. Coarse median spaces were introduced in Bow13a as an attempt to formulate a coarse notion of nonpositive curvature. They have recently received a lot of attention 
Bow13b, Hae16a, Zei16, ŠW17, NWZ18b, NWZ18a, ANWZ18] and proved instrumental to striking results such as [Hae16b, BHS17b].

A finitely generated group is said to be coarse median if its Cayley graphs are coarse median spaces; this property is independent of the chosen finite generating set. Examples of coarse median groups of finite rank include hyperbolic groups, mapping class groups, uniform lattices in products of rank-one simple Lie groups, fundamental groups of closed irreducible 3manifolds not modelled on Nil or Sol and all cubulated groups - in particular, right-angled Coxeter and Artin groups. More generally, if a group is a hierarchically hyperbolic space (HHS), it is coarse median of finite rank BHS17a, BHS19, Bow18].

We will be mainly interested in equivariantly coarse median groups. If we view coarse median groups as a generalisation of groups that are HHS, equivariantly coarse median groups generalise hierarchically hyperbolic groups $(H H G)$. In particular, all the examples above are also equivariantly coarse median of finite rank (with the exception of some graph manifold groups).

More precisely, we say that a group $H$ is equivariantly coarse median if it is equipped with a finite generating set $S \subseteq H$ and a coarse median $\mu: H^{3} \rightarrow H$ Bow13a such that $d_{S}(\mu(h a, h b, h c), h \mu(a, b, c)) \leq C<+\infty$ for all elements $h, a, b, c \in H$; here $d_{S}$ denotes the word metric induced by $S$. Note that this definition does not depend on the choice of $S$. Equivariantly coarse median groups have already been considered in [Zei16] under the name of "uniformly left-invariant coarse median structures".

If $H$ is a coarse median group of finite rank, every asymptotic cone of $H$ is endowed with a canonical, bi-Lipschitz equivalent median metric [Zei16]. As an example, in asymptotic cones of mapping class groups the median geodesics are limits of hierarchy paths BDS11a, BDS11b. When $H$ is equivariantly coarse median, the median metric on each asymptotic cone is preserved by the action of the ultrapower of $H$.

Given a group $\Gamma$ and an infinite sequence of pairwise non-conjugate homomorphisms $\Gamma \rightarrow H$, we can apply the Bestvina-Paulin construction Bes88. Pau88. The result is an isometric action on a median space $X$ with unbounded orbits; this is obtained as the canonical median space bi-Lipschitz equivalent to an asymptotic cone of $H$. Along with Theorem C, this implies:

Corollary E. Let $H$ be an equivariantly coarse median group of finite rank. Let $\Gamma$ be as in the second part of Theorem $C$. There exist only finitely many pairwise non-conjugate homomorphisms $\Gamma \rightarrow H$.

When $\Gamma$ is a uniform irreducible lattice in a product $G$ of rank-one simple Lie groups, we can take $\Gamma=H$ and Corollary E provides an alternative proof that $\operatorname{Out}(\Gamma)$ is finite, without relying on Margulis' superrigidity, nor on the structure of the normaliser of $\Gamma$ in $G$ (Lemma II.6.3 in [Mar91]).

If instead $\Gamma$ is a lattice in a product of higher-rank, simple Lie groups, compare Theorem 5.6 in [Zei16] and Corollary D in [Hae16b]. Also see Theorem 4.1 in [BF14]. 
We remark that Corollary E can be strengthened significantly if $H$ acts freely on a complete, finite rank median space. Indeed, the following is an immediate consequence of Theorem $\mathrm{F}$ in [Fio18.

Proposition F. Let $H$ be a group admitting a free action on a complete, finite rank median space $X$. Suppose that every action $\Gamma \curvearrowright X$ is Roller elementary. Then every homomorphism $\Gamma \rightarrow H$ factors through a virtually abelian subgroup of $H$.

Proposition $\mathrm{F}$ applies for instance to the case when $\Gamma$ has no non-abelian free subgroups, has property $H_{F D}$ (see Theorem $\mathrm{G}$ below) or satisfies the hypotheses of the first part of Theorem C. In particular, if $\Gamma$ is an irreducible lattice in a connected, higher rank, semisimple Lie group, every homomorphism $\Gamma \rightarrow H$ has finite image.

This should motivate a certain interest in groups acting freely on complete, finite rank median spaces. If a group acts freely on a finite dimensional CAT(0) cube complex, it clearly falls into this class; however, it is unclear at this stage whether these are the only finitely generated examples. See CRK15 for partial results in this direction.

Note that the infinitely generated group $\mathbb{Q}$ admits a proper action on a rank two median space, namely the product of a simplicial tree and the real line (Example II.7.13 in [BH99]). However, since $\mathbb{Q}$ is a divisible group, all its elements must act elliptically on any (possibly infinite-dimensional) CAT(0) cube complex Hag07.

Even within finitely generated groups, actions on median spaces tend to be more flexible than actions on CAT $(0)$ cube complexes. For every group $H$, we can consider $\operatorname{dim}_{f m} H$, i.e. the minimum rank of a complete median space $X$ admitting a free action of $H$; if $H$ does not act freely on any complete median space, we set $\operatorname{dim}_{f m} H:=-1$. Restricting to CAT(0) cube complexes, we can similarly define $\operatorname{dim}_{f c} H$ and, if we only consider (metrically) proper actions, we obtain $\operatorname{dim}_{p m} H$ and $\operatorname{dim}_{p c} H$. Thus, $\operatorname{dim}_{p c} \mathbb{Q}=\operatorname{dim}_{f c} \mathbb{Q}=-1$, while $\operatorname{dim}_{p m} \mathbb{Q}=2$ and $\operatorname{dim}_{f m} \mathbb{Q}=1$ (the latter since $\mathbb{Q}<\mathbb{R}$ ).

We remark that $1 \leq \operatorname{dim}_{f m} H<\operatorname{dim}_{c m} H$ and $1 \leq \operatorname{dim}_{p m} H<\operatorname{dim}_{p m} H$ for many finitely generated groups $H$. For instance, $\operatorname{dim}_{f c} H=1$ if and only if $H$ is free. On the other hand, by work of E. Rips, $\operatorname{dim}_{f m} H=1$ if and only if $H$ is a free product of free abelian and surface groups (excluding a few nonorientable surfaces); see e.g. Theorem 9.8 in [BF95. One can use the same observation to construct free actions of various right-angled Artin groups on median spaces of rank strictly lower than the dimension of the Salvetti complex.

Considering more general actions, we mention that there exist finitely generated groups admitting actions on real trees with unbounded orbits, but whose actions on finite dimensional $\mathrm{CAT}(0)$ cube complexes must all have global fixed points. An example is provided by the group $L$ in Section 2 of [Min16]; the fixed point property for actions on finite dimensional cube 
complexes can easily be derived from the same property of Thompson's group $V$ Gen19, Kat18.

1.5. Shalom's property $H_{F D}$ and random groups. Theorem $\mathrm{A}$ also allows us to prove that various (non-amenable) groups do not have property $H_{F D}$. The latter was introduced in [Sha04]: a topological group $G$ has property $H_{F D}$ if every unitary representation $\pi$ with $\overline{H_{c}^{1}}(G, \pi) \neq 0$ has a finite dimensional subrepresentation.

Property $H_{F D}$ is trivially satisfied by every locally compact group with property (T) Del77, but also, at the opposite end of the universe of groups, by a large class of amenable groups. This includes polycyclic groups, lamplighter groups and all connected, locally compact, amenable groups [Sha04, Mar06, EO18]. An example of an amenable group without $H_{F D}$ is provided by the wreath product $\mathbb{Z} \imath \mathbb{Z}$ [Sha04]. We prove the following:

Theorem G. If $G$ has property $H_{F D}$, every isometric action of $G$ on a complete, finite rank median space is Roller elementary.

Corollary H. Let $\Gamma$ be a discrete group with property $H_{F D}$. If $\Gamma$ acts freely and cocompactly on a $\mathrm{CAT}(0)$ cube complex $X$, then $\Gamma$ is virtually abelian.

Property $H_{F D}$ has been studied almost exclusively within the class of amenable groups, where it happens to be a quasi-isometry invariant Sha04. It was a key ingredient (implicitly, or explicitly) in recent more elementary proofs of Gromov's theorem on groups of polynomial growth Kle10, Oza18. It has moreover interesting applications to the study of quasi-isometric embeddings into Hilbert spaces dCTV07.

Property $H_{F D}$ is inherited by uniform lattices and is stable under direct products and central extensions Sha04. Being satisfied by groups that fall into two extremely different classes, namely amenable and Kazhdan groups, it is reasonable to expect a wide variety of groups with property $H_{F D}$. However, it seems that no answer is known to the following question.

Question. Does every finitely generated group with property $H_{F D}$ virtually split as a direct product of an amenable group and finitely many groups with property $(\mathrm{T})$ ? Does every word hyperbolic group with property $H_{F D}$ also satisfy property $(\mathrm{T})$ ?

Corollary $\mathrm{H}$ and the results of [OW11 imply that random groups at low density do not satisfy $H_{F D}$.

Corollary I. With overwhelming probability, random groups at density $d<\frac{1}{6}$ in Gromov's density model do not have property $H_{F D}$.

Note however that, at density $d>\frac{1}{3}$, random groups are Kazhdan $[\dot{\mathrm{Z}} 03$, KK13, hence satisfy property $H_{F D}$.

Acknowledgements. The author warmly thanks Brian Bowditch, Pierre-Emmanuel Caprace, Indira Chatterji, Yves Cornulier, Thomas Delzant, Mark Hagen, Masato Mimura, Narutaka Ozawa, Romain Tessera, Pierre 
Pansu, Alain Valette for helpful conversations. The author expresses special gratitude to Cornelia Druţu and Talia Fernós for their interest and encouragement throughout the writing of this paper. The author also wishes to thank the anonymous referee for many valuable comments.

This work was undertaken at the Mathematical Sciences Research Institute in Berkeley during the Fall 2016 program in Geometric Group Theory, where the author was supported by the National Science Foundation under Grant no. DMS-1440140 and by the GEAR Network. Part of this work was also carried out at the Isaac Newton Institute for Mathematical Sciences, Cambridge, during the programme "Non-positive curvature, group actions and cohomology" and was supported by EPSRC grant no. EP/K032208/1. The author was also supported by the Clarendon Fund and the Merton Moussouris Scholarship.

\section{Preliminaries.}

2.1. Median spaces and median algebras. Let $X$ be a metric space. Given points $x, y \in X$, the interval $I(x, y)$ is the set of points $z \in X$ that lie between $x$ and $y$, i.e. that satisfy $d(x, y)=d(x, z)+d(z, y)$. We say that $X$ is a median space if for all $x, y, z \in X$ there exists a unique point $m(x, y, z)$ that lies in $I(x, y) \cap I(y, z) \cap I(z, x)$. The median map $m: X^{3} \rightarrow X$ that we obtain this way endows $X$ with a structure of median algebra (see $\S 1.1$ in [Rol98]). Most definitions in the theory of median spaces can also be given for arbitrary median algebras; we will follow this approach in introducing the necessary notions. The reader can consult e.g. Rol98, Nic08, CDH10, Bow13a, Bow16, Fio17, Fio18 for more background on median spaces and algebras.

In a median space, $I(x, y)=\{z \in I(x, y) \mid z=m(x, y, z)\}$; this can be taken as a definition of intervals in general median algebras. If $(M, m)$ is a median algebra, we say that a subset $C \subseteq M$ is convex if $I(x, y) \subseteq C$ whenever $x, y \in C$. The intersection of a finite family of pairwise intersecting convex sets is always nonempty; this is known as Helly's Theorem, see Theorem 2.2 in Rol98.

A subset $\mathfrak{h} \subseteq M$ is a halfspace if both $\mathfrak{h}$ and $\mathfrak{h}^{*}:=M \backslash \mathfrak{h}$ are convex; we will denote the set of halfspaces of $M$ by $\mathscr{H}(M)$, or simply by $\mathscr{H}$ when there is no ambiguity. Halfspaces $\mathfrak{h}, \mathfrak{k}$ are said to be transverse if no two distinct elements of the set $\left\{\mathfrak{h}, \mathfrak{h}^{*}, \mathfrak{k}, \mathfrak{k}^{*}\right\}$ are comparable in the poset $(\mathscr{H}, \subseteq)$. Equivalently, the intersections $\mathfrak{h} \cap \mathfrak{k}, \mathfrak{h} \cap \mathfrak{k}^{*}, \mathfrak{h}^{*} \cap \mathfrak{k}, \mathfrak{h}^{*} \cap \mathfrak{k}^{*}$ are all nonempty.

Given $A \subseteq \mathscr{H}$, we write $A^{*}$ for $\left\{\mathfrak{h} \in \mathscr{H} \mid \mathfrak{h}^{*} \in A\right\}$. A subset $\sigma \subseteq \mathscr{H}$ is said to be an ultrafilter if any two halfspaces in $\sigma$ intersect and $\mathscr{H}=\sigma \sqcup \sigma^{*}$. For instance, for each $x \in M$ the set $\sigma_{x}:=\{\mathfrak{h} \in \mathscr{H} \mid x \in \mathfrak{h}\}$ is an ultrafilter.

Given subsets $A, B \subseteq M$, we write $\mathscr{H}(A \mid B):=\left\{\mathfrak{h} \in \mathscr{H} \mid B \subseteq \mathfrak{h}, A \subseteq \mathfrak{h}^{*}\right\}$ and $\sigma_{A}:=\mathscr{H}(\emptyset \mid A)$; we refer to sets of the form $\mathscr{H}(x \mid y), x, y \in M$, as halfspace intervals. If $C, C^{\prime} \subseteq M$ are disjoint and convex, the set $\mathscr{H}\left(C \mid C^{\prime}\right)$ 
is nonempty, see Theorem 2.7 in [Rol98]. In particular $\sigma_{x}=\sigma_{y}$ if and only if the points $x, y \in M$ coincide.

A subset $\Omega \subseteq \mathscr{H}$ is inseparable if, whenever $\mathfrak{j} \in \mathscr{H}$ satisfies $\mathfrak{h} \subseteq \mathfrak{j} \subseteq \mathfrak{k}$ for $\mathfrak{h}, \mathfrak{k} \in \Omega$, we have $\mathfrak{j} \in \Omega$. Given a subset $A \subseteq \mathscr{H}$, its inseparable closure is the smallest inseparable subset of $\mathscr{H}$ that contains $A$; it coincides with the union of the sets $\mathscr{H}\left(\mathfrak{k}^{*} \mid \mathfrak{h}\right)$, for $\mathfrak{h}, \mathfrak{k} \in A$.

A wall is a set of the form $\mathfrak{w}=\left\{\mathfrak{h}, \mathfrak{h}^{*}\right\}$, with $\mathfrak{h} \in \mathscr{H}$; we say that $\mathfrak{h}$ and $\mathfrak{h}^{*}$ are the sides of $\mathfrak{w}$. The wall $\mathfrak{w}$ separates subsets $A, B \subseteq M$ if either $\mathfrak{h}$ or $\mathfrak{h}^{*}$ lies in $\mathscr{H}(A \mid B)$; we denote by $\mathscr{W}(A \mid B)=\mathscr{W}(B \mid A)$ the set of walls separating $A$ and $B$ and by $\mathscr{W}(M)$, or simply $\mathscr{W}$, the set of all walls of the median algebra $M$. A wall is contained in a halfspace $\mathfrak{k}$ if one of its sides is; a wall $\mathfrak{w}$ is contained in disjoint halfspaces $\mathfrak{k}_{1}, \mathfrak{k}_{2}$ if and only if $\mathfrak{k}_{2}=\mathfrak{k}_{1}^{*}$ and $\mathfrak{w}=\left\{\mathfrak{k}_{1}, \mathfrak{k}_{2}\right\}$. If a side of the wall $\mathfrak{w}_{1}$ is transverse to a side of the wall $\mathfrak{w}_{2}$, we say that $\mathfrak{w}_{1}$ and $\mathfrak{w}_{2}$ are transverse.

The rank of the median algebra $M$ is the maximum cardinality of a set of pairwise transverse walls; various alternative (and equivalent) definitions of the rank can be found in Proposition 6.2 of [Bow13a]. We remark that $M$ has rank zero if and only if it consists of a single point.

If $X$ is a connected median space, its rank coincides with the supremum of the topological dimensions of its locally compact subsets - even when either of the two quantities is infinite. See Theorem 2.2 and Lemma 7.6 in [Bow13a] for one inequality and Proposition 5.6 in [Bow16] for the other.

When $X$ is complete, connected and finite rank, $X$ is bi-Lipschitz equivalent to a canonical CAT(0) space $\widehat{X}$ Bow16. The visual boundary of $\widehat{X}$ is finite dimensional by Proposition 2.1 in [CL10]. Every isometry of $X$ extends to an isometry of $\widehat{X}$ yielding a homomorphism Isom $X \hookrightarrow$ Isom $\widehat{X}$. Every convex subset of $X$ is also convex in $\widehat{X}$; the converse is not true: the euclidean convex hull of the points $(1,0,0),(0,1,0)$ and $(1,1,1)$ in the cube $[0,1]^{3}$ is not even a median subalgebra.

Halfspaces in finite rank median spaces are fairly well-behaved. See Corollary 2.23 and Proposition 2.26 in [Fio17] for a proof of the following:

Proposition 2.1. Let $X$ be a complete median space of finite rank $r$. Every halfspace is either open or closed (possibly both). Moreover, if $\mathfrak{h}_{1} \supsetneq \ldots \supsetneq \mathfrak{h}_{k}$ is a chain of halfspaces with $\overline{\mathfrak{h}_{1}^{*}} \cap \overline{\mathfrak{h}_{k}} \neq \emptyset$, we have $k \leq 2 r$.

The following is a simple but extremely useful observation: given ultrafilters $\sigma_{1}, \sigma_{2} \subseteq \mathscr{H}(M)$ and $\mathfrak{h}, \mathfrak{k} \in \sigma_{1} \backslash \sigma_{2}$, we either have $\mathfrak{h} \subseteq \mathfrak{k}$, or $\mathfrak{k} \subseteq \mathfrak{h}$, or $\mathfrak{h}$ and $\mathfrak{k}$ are transverse. Along with Dilworth's Theorem [Dil50] this yields the following.

Lemma 2.2. Let $M$ be a median algebra of finite rank $r$ and let $\sigma_{1}, \sigma_{2} \subseteq \mathscr{H}$ be ultrafilters.

(1) We can decompose $\sigma_{1} \backslash \sigma_{2}=\mathcal{C}_{1} \sqcup \ldots \sqcup \mathcal{C}_{k}$, where $k \leq r$ and each $\mathcal{C}_{i}$ is nonempty and totally ordered by inclusion. 
(2) Every infinite subset of $\sigma_{1} \backslash \sigma_{2}$ contains an infinite subset that is totally ordered by inclusion.

If $C \subseteq M$ is a subset and $x \in M$, a gate for $(x, C)$ is a point $y \in C$ such that $y \in I(x, z)$ for every $z \in C$; gates are unique when they exist. If a gate exists for every point of $M$, we say that $C$ is gate-convex; in this case we can define a gate-projection $\pi_{C}: M \rightarrow C$ by associating to each point of $M$ the unique gate. The gate-projection to $C$ is a morphism of median algebras and satisfies $\mathscr{W}(x \mid C)=\mathscr{W}\left(x \mid \pi_{C}(x)\right)$ for every $x \in M$; see Proposition 2.1 and Lemma 2.4 in [Fio17].

Gate-convex subsets are always convex, but the converse is not always true. For every $y, z \in M$, the interval $I(y, z)$ is gate-convex with gateprojection $x \mapsto m(x, y, z)$. The following is a summary of Lemma 2.2, Proposition 2.3 and Lemma 2.4 in [Fio17.

Proposition 2.3. Let $C, C^{\prime} \subseteq M$ be gate-convex.

(1) The sets $\left\{\mathfrak{h} \in \mathscr{H}(M) \mid \mathfrak{h} \cap C \neq \emptyset, \mathfrak{h}^{*} \cap C \neq \emptyset\right\},\left\{\pi_{C}^{-1}(\mathfrak{h}) \mid \mathfrak{h} \in \mathscr{H}(C)\right\}$ and $\mathscr{H}(C)$ are all naturally in bijection.

(2) There exists a pair of gates, i.e. a pair $\left(x, x^{\prime}\right)$ of points $x \in C$ and $x^{\prime} \in C^{\prime}$ such that $\pi_{C}\left(x^{\prime}\right)=x$ and $\pi_{C^{\prime}}(x)=x^{\prime}$. In particular, we have $\mathscr{H}\left(x \mid x^{\prime}\right)=\mathscr{H}\left(C \mid C^{\prime}\right)$.

(3) The set $\pi_{C}\left(C^{\prime}\right)$ is gate-convex with gate-projection $\pi_{C} \circ \pi_{C^{\prime}}$. Moreover, $\pi_{C} \circ \pi_{C^{\prime}} \circ \pi_{C}=\pi_{C} \circ \pi_{C^{\prime}}$.

(4) If $C \cap C^{\prime} \neq \emptyset$, we have $\pi_{C}\left(C^{\prime}\right)=C \cap C^{\prime}$ and $\pi_{C} \circ \pi_{C^{\prime}}=\pi_{C^{\prime}} \circ \pi_{C}$. In particular, if $C^{\prime} \subseteq C$, we have $\pi_{C^{\prime}}=\pi_{C^{\prime}} \circ \pi_{C}$.

A median algebra $(M, m)$ endowed with a Hausdorff topology is said to be a topological median algebra if the median map $m: M^{3} \rightarrow M$ is continuous; here we equip $M^{3}$ with the product topology. Median spaces always provide topological median algebras; indeed, the median map $m$ is 1-Lipschitz in that case (see Corollary 2.15 in [CDH10]).

In compact median algebras and complete median spaces, a subset is gateconvex if and only if it is closed and convex; moreover, gate-projections are continuous. In median spaces, gate-projections are even 1-Lipschitz. See Lemmas 2.6 and 2.7 in [Fio17] and Lemma 2.13 in [CDH10] for details.

Let now $X$ be a complete, finite rank median space. In Section 3 of [Fio17, we endowed the set $\mathscr{H}$ with a $\sigma$-algebra $\widehat{\mathscr{B}}$ and a measure $\widehat{\nu}_{X}$ (usually denoted just $\widehat{\nu}$ ). Unlike [Fio17], here we simply refer to the elements of $\widehat{\mathscr{B}}$ as measurable sets.

Note that in general $\widehat{\mathscr{B}}$ and $\widehat{\nu}$ differ from their counterparts in [CDH10, in that $\widehat{\mathscr{B}}$ contains more measurable and null sets. More precisely, a subset $E \subseteq \mathscr{H}$ is measurable/null if and only if every intersection with a halfspace interval is measurable/null. This results in the following useful properties.

The map $*: \mathscr{H} \rightarrow \mathscr{H}$ sending each halfspace to its complement is measure preserving. Every inseparable subset of $\mathscr{H}$ is measurable (Lemma 3.9 in [Fio17]); in particular, all ultrafilters are measurable and, for all $x, y \in X$, we 
have $\widehat{\nu}\left(\sigma_{x} \triangle \sigma_{y}\right)=d(x, y)$. Almost every halfspace $\mathfrak{h} \in \mathscr{H}$ is thick, i.e. both $\mathfrak{h}$ and $\mathfrak{h}^{*}$ have nonempty interior (Corollary 3.7 in [Fio17]). The next result, moreover, is Corollary 3.11 in [Fio17.

Proposition 2.4. Let $X$ be a complete, finite rank median space and $\sigma \subseteq \mathscr{H}$ an ultrafilter such that $\widehat{\nu}\left(\sigma \triangle \sigma_{x}\right)<+\infty$ for some $x \in X$. There exists $y \in X$ such that $\widehat{\nu}\left(\sigma \triangle \sigma_{y}\right)=0$.

As a consequence, $X$ can be equivalently described as the collection of all ultrafilters on $\mathscr{H}$ that satisfy $\widehat{\nu}\left(\sigma \triangle \sigma_{x}\right)<+\infty$ for some $x \in X$; we identify ultrafilters whose symmetric difference is $\widehat{\nu}$-null. Considering instead the space of all ultrafilters on $\mathscr{H}$ we obtain a set $\bar{X}$ in which $X$ embeds. A structure of median algebra can be defined on $\bar{X}$ by setting

$$
m\left(\sigma_{1}, \sigma_{2}, \sigma_{3}\right):=\left(\sigma_{1} \cap \sigma_{2}\right) \cup\left(\sigma_{2} \cap \sigma_{3}\right) \cup\left(\sigma_{3} \cap \sigma_{1}\right) .
$$

We endow $\bar{X}$ with a topology such that ultrafilters $\sigma_{n} \subseteq \mathscr{H}$ converge to $\sigma \subseteq \mathscr{H}$ if and only if $\lim \sup \left(\sigma_{n} \triangle \sigma\right)$ is $\widehat{\nu}$-null; see Section 4.2 and, in particular, Lemma 4.16 in [Fio17. We refer to $\bar{X}$ as the Roller compactification.

Note that the identity map of $X$ canonically extends to a homeomorphism between $\bar{X}$ and the Busemann compactification of $X$ (Proposition 4.21 in [Fio17]). The difference in terminology is motivated by the additional median structure. The following is part of Proposition 4.14 in [Fio17.

Proposition 2.5. The Roller compactification $\bar{X}$ is a compact topological median algebra. The inclusion $X \hookrightarrow \bar{X}$ is a continuous morphism of median algebras with dense, convex image.

If $X$ is connected and locally compact, $X$ is open in $\bar{X}$ and the inclusion $X \hookrightarrow \bar{X}$ is a homeomorphism onto its image (Proposition 4.20 in [Fio17]). This however fails in general. The Roller boundary is defined as $\partial X:=\bar{X} \backslash X$.

A point of $\bar{X}$ can often be represented by several distinct ultrafilters with null symmetric differences. However, for each $\xi \in \bar{X}$ there is a unique preferred ultrafilter $\sigma_{\xi}$ representing $\xi$ (Lemma 4.15 in [Fio17]). This should be seen as a generalisation of the ultrafilters $\sigma_{x}$ when $x \in X$.

We can extend each halfspace $\mathfrak{h}$ of $X$ to a halfspace $\tilde{\mathfrak{h}}$ of $\bar{X}$ such that $\widetilde{\mathfrak{h}} \cap X=\mathfrak{h}$; indeed, it suffices to define $\widetilde{\mathfrak{h}}:=\left\{\xi \in \bar{X} \mid \mathfrak{h} \in \sigma_{\xi}\right\}$. When $\xi, \eta \in \bar{X}$, we save the notation $\mathscr{H}(\xi \mid \eta)$ for the set $\sigma_{\eta} \backslash \sigma_{\xi} \subseteq \mathscr{H}(X)$, instead of the analogous subset of $\mathscr{H}(\bar{X})$.

If $Y \subseteq X$ is a closed median subalgebra, the restriction of the metric of $X$ turns $Y$ into a complete median space with $\operatorname{rank}(Y) \leq \operatorname{rank}(X)$; moreover:

Lemma 2.6. There is a canonical morphism of median algebras $\iota_{Y}: \bar{Y} \hookrightarrow \bar{X}$.

Proof. We write $\mathscr{H}_{Y}:=\left\{\mathfrak{h} \in \mathscr{H}(X) \mid \mathfrak{h} \cap Y \neq \emptyset, \mathfrak{h}^{*} \cap Y \neq \emptyset\right\}$; intersecting with $Y$ gives a map $p: \mathscr{H}_{Y} \rightarrow \mathscr{H}(Y)$. Lemma 6.5 in [Bow13a] implies that $p$ is surjective. Thus, for every ultrafilter $\sigma \subseteq \mathscr{H}(Y)$, there is a unique ultrafilter $\sigma^{\prime} \subseteq \mathscr{H}(X)$ such that $\sigma_{Y} \subseteq \sigma^{\prime}$ and $p\left(\sigma^{\prime} \cap \mathscr{H}_{Y}\right)=\sigma$. Applying this to preferred ultrafilters yields the required embedding. 
Given ultrafilters $\sigma_{1}, \sigma_{2} \subseteq \mathscr{H}$, we set $d\left(\sigma_{1}, \sigma_{2}\right):=\widehat{\nu}\left(\sigma_{1} \triangle \sigma_{2}\right)$. We refer to $d$ as the extended metric on $\bar{X}$ as it satisfies all the axioms of a metric, even though the value $+\infty$ is allowed. Note that, for points of $X$, this is the same as the original median metric on $X$.

A component $Z \subseteq \bar{X}$ is a maximal set of points having pairwise finite distances. Components are convex subsets of $\bar{X}$ (Proposition 4.19 in [Fio17]). One component always coincides with $X \subseteq \bar{X}$; all other components are contained in $\partial X$. For the following, see Propositions 4.23 and 4.29 in [Fio17].

Proposition 2.7. Let $X$ be a complete median space of finite rank $r$. Let $Z \subseteq \partial X$ be a component and let d denote the extended metric on $\bar{X}$.

(1) The metric space $(Z, d)$ is a complete median space of rank at most $r-1$.

(2) Every thick halfspace of $Z$ is of the form $\widetilde{\mathfrak{h}} \cap Z$ for a unique $\mathfrak{h} \in \mathscr{H}$.

If $C \subseteq X$ is closed and convex, the closure of $C$ in $\bar{X}$ is gate-convex and naturally identified with the Roller compactification of $C$ (see Proposition 4.14 in [Fio17]); thus the notation $\bar{C}$ is not ambiguous. We denote by $\pi_{C}: \bar{X} \rightarrow \bar{C}$ the corresponding gate-projection; it extends the usual gateprojection $X \rightarrow C$. If $\sigma \subseteq \mathscr{H}(X)$ is an ultrafilter representing the point $\xi \in \bar{X}$, the set $\sigma \cap \mathscr{H}(C)$ is an ultrafilter on $\mathscr{H}(C)$ and represents $\pi_{C}(\xi)$.

Similarly, if $Z \subseteq \partial X$ is a component, the closure of $Z$ in $\bar{X}$ is gate-convex and naturally identified with the Roller compactification $\bar{Z}$ (Proposition 4.28 in [Fio17]). The gate-projection $\pi_{Z}: \bar{X} \rightarrow \bar{Z}$ satisfies $\pi_{Z}(X) \subseteq Z$. In terms of ultrafilters, $\pi_{Z}$ takes the point of $\bar{X}$ represented by $\sigma \subseteq \mathscr{H}(X)$ to the point of $\bar{Z}$ represented by $\sigma \cap \mathscr{H}(Z) \subseteq \mathscr{H}(Z)$. The intersection makes sense as, by part 2 of Proposition 2.7, almost every halfspace of $Z$ arises from a halfspace of $X$.

Let now $\Gamma$ be a group and $\Gamma \curvearrowright X$ an action by isometries.

Definition 2.8. We say that $\Gamma \curvearrowright X$ is Roller elementary if there exists a finite orbit within $\bar{X}$. The action is Roller minimal if $\operatorname{rank}(X) \geq 1$ and $\Gamma$ does not preserve any proper, closed, convex subset $C \subseteq \bar{X}$.

Roller elementarity implies - but is in general much stronger than - the existence of a finite orbit in the visual compactification of $\widehat{X}$. When $X$ is a CAT(0) cube complex, an action is Roller minimal if and only if, in the terminology of [CS11], it is essential and does not fix any point in the visual boundary of $\widehat{X}$. For these statements, see Proposition 5.2 in [Fio18].

Neither Roller elementarity, nor Roller minimality implies the other one. However, Roller minimal actions naturally arise from Roller nonelementary ones (Proposition 5.5 in [Fio18]):

Proposition 2.9. Let $X$ be a complete, finite rank median space with an isometric action $\Gamma \curvearrowright X$. Either $\Gamma \curvearrowright \bar{X}$ fixes a point or there exist a $\Gamma$ invariant component $Z \subseteq \bar{X}$ and a $\Gamma$-invariant, closed, convex subset $C \subseteq Z$ such that $\Gamma \curvearrowright C$ is Roller minimal. 
If $Z \subseteq \bar{X}$ is a component, any $\Gamma$-invariant, closed, convex subset $C \subseteq Z$ gives rise to a measurable decomposition $\mathscr{H}=\mathscr{H}_{C} \sqcup\left(\sigma_{C} \cup \sigma_{C}^{*}\right)$. Here, we have introduced the sets $\mathscr{H}_{C}:=\left\{\mathfrak{h} \in \mathscr{H} \mid C \cap \widetilde{\mathfrak{h}} \neq \emptyset, C \cap \widetilde{\mathfrak{h}}^{*} \neq \emptyset\right\}$ and $\sigma_{C}:=\{\mathfrak{h} \in \mathscr{H} \mid C \subseteq \widetilde{\mathfrak{h}}\}$. Note that, by part 2 of Proposition 2.7, the measure spaces $\left(\mathscr{H}_{C}, \widehat{\nu}_{X}\right)$ and $\left(\mathscr{H}(C), \widehat{\nu}_{C}\right)$ are isomorphic.

We say that an action $\Gamma \curvearrowright X$ is without wall inversions if there do not exist $g \in \Gamma$ and $\mathfrak{h} \in \mathscr{H}$ such that $g \mathfrak{h}=\mathfrak{h}^{*}$. By Proposition 2.1, any action on a connected, complete, finite rank median space is without wall inversions. The following appears as Corollary 5.4 in [Fio18]; compare with [CS11].

Proposition 2.10. Let $X$ be a complete, finite rank median space with thick halfspaces $\mathfrak{h} \subseteq \mathfrak{k}$. Let $\Gamma \curvearrowright X$ be a Roller minimal action without wall inversions.

(1) There exists $g \in \Gamma$ such that $g \mathfrak{h}^{*} \subsetneq \mathfrak{h}$ and $d\left(g \mathfrak{h}^{*}, \mathfrak{h}^{*}\right)>0$.

(2) There exists $g \in \Gamma$ such that $g \mathfrak{k} \subsetneq \mathfrak{h} \subseteq \mathfrak{k}$ and $d\left(g \mathfrak{k}, \mathfrak{h}^{*}\right)>0$.

When $G$ is a topological group, all isometric actions $G \curvearrowright X$ will be implicitly required to have continuous orbit maps. Equivalently, the homomorphism $G \rightarrow$ Isom $X$ is continuous, where we endow Isom $X$ with the topology of pointwise convergence. We remark that Isom $X$ is a Hausdorff, sequentially complete topological group as soon as $X$ is complete.

If $\left(M_{1}, m_{1}\right)$ and $\left(M_{2}, m_{2}\right)$ are median algebras, the product median algebra is defined as $\left(M_{1} \times M_{2}, m\right)$, where $m=\left(m_{1} \circ p_{1}, m_{2} \circ p_{2}\right)$; here $p_{i}$ denotes the projection onto the $i$-th factor. If $\left(X_{1}, d_{1}\right)$ and $\left(X_{2}, d_{2}\right)$ are median spaces, we endow the product $X_{1} \times X_{2}$ with the $\ell^{1}$ metric, namely $d=d_{1} \circ p_{1}+d_{2} \circ p_{2}$. The median algebra associated to the median space $\left(X_{1} \times X_{2}, d\right)$ is just the product median algebra arising from $X_{1}$ and $X_{2}$.

A median space $X$ is said to be irreducible if it is not isometric to any nontrivial product $X_{1} \times X_{2}$ of median spaces. The structure of reducible median spaces is described by the following result.

Proposition 2.11. Let $X_{1}, \ldots, X_{k}$ be irreducible, complete, finite rank median spaces; consider the product $X=X_{1} \times \ldots \times X_{k}$.

(1) We have a measurable partition $\mathscr{H}(X)=\mathscr{H}_{1} \sqcup \ldots \sqcup \mathscr{H}_{k}$, where each $\mathscr{H}_{i}$ is canonically identified with $\mathscr{H}\left(X_{i}\right)$. If $\mathfrak{h} \in \mathscr{H}_{i}$ and $\mathfrak{k} \in \mathscr{H}_{j}$ with $i \neq j$, the halfspaces $\mathfrak{h}$ and $\mathfrak{k}$ are transverse.

(2) Every isometry of $X$ permutes the members of the partition. The product Isom $X_{1} \times \ldots \times$ Isom $X_{k}$ sits inside Isom $X$ as the open, finite index subgroup preserving the splitting $X=X_{1} \times \ldots \times X_{k}$.

(3) Every closed, convex subset $C \subseteq X$ is of the form $C_{1} \times \ldots \times C_{k}$, where each $C_{i}$ is a closed convex subset of $X_{i}$.

(4) The Roller compactification $\bar{X}$ is naturally identified with the product median algebra $\overline{X_{1}} \times \ldots \times \overline{X_{k}}$, endowed with the product topology.

Proof. Let $p_{i}: X \rightarrow X_{i}$ denote the projection onto the $i$-th factor. Given $\mathfrak{h} \in \mathscr{H}\left(X_{i}\right)$, we have $p_{i}^{-1}(\mathfrak{h}) \in \mathscr{H}(X)$; let $\mathscr{H}_{i}$ be the set of halfspaces of $X$ that arise this way. With this notation, part 1 is Proposition 2.10 in [Fio18]. 
Regarding part 2, Proposition 2.12 in [Fio18] shows that each $g \in$ Isom $X$ permutes the sets $\mathscr{H}_{i}$, or equivalently the factors $X_{i}$. Moreover, the subgroup of isometries preserving each $\mathscr{H}_{i}$ (call it $H$ ) has finite index in Isom $X$ and is naturally identified with the product Isom $X_{1} \times \ldots \times$ Isom $X_{k}$. We are left to prove that $H$ is open in Isom $X$.

Choose distinct points $x_{i}, y_{i} \in X_{i}$ and a real number $\epsilon>0$ such that $2 \epsilon<d\left(x_{i}, y_{i}\right)$ for all $i$. Let $x \in X$ be the point with coordinates $x_{i}$; we also introduce the points $z_{i} \in X$ such that $p_{j}\left(z_{i}\right)=x_{j}$ for all $i \neq j$ and $p_{i}\left(z_{i}\right)=y_{i}$. We will show that an isometry $F \in$ Isom $X$ lies in $H$ as soon as $d(F(x), x)<\epsilon$ and $d\left(F\left(z_{i}\right), z_{i}\right)<\epsilon$ for all $i$. This will yield that $H$ is open.

Suppose for the sake of contradiction that such an isometry $F$ does not lie in $H$; in particular, there exist indices $i \neq j$ such that $F\left(\mathscr{H}_{i}\right)=\mathscr{H}_{j}$. Since $X_{i}$ can be realised via ultrafilters on $\mathscr{H}_{i}=\mathscr{H}\left(X_{i}\right)$, this naturally induces an isometry $f: X_{i} \rightarrow X_{j}$; cf. Corollary 3.13 in [Fio17. Observe that $p_{j} \circ F=f \circ p_{i}$. Thus, we have $f\left(x_{i}\right)=f p_{i}(x)=p_{j} F(x)$ and

$$
d\left(x_{j}, f\left(x_{i}\right)\right)=d\left(p_{j}(x), p_{j} F(x)\right) \leq d(x, F(x))<\epsilon .
$$

Similarly, $d\left(x_{j}, f\left(y_{i}\right)\right)=d\left(p_{j}\left(z_{i}\right), p_{j} F\left(z_{i}\right)\right) \leq d\left(z_{i}, F\left(z_{i}\right)\right)<\epsilon$. We conclude that $d\left(x_{i}, y_{i}\right)=d\left(f\left(x_{i}\right), f\left(y_{i}\right)\right)<2 \epsilon$, a contradiction.

We now prove part 3 . Irreducibility of the factors plays no role here, so it suffices to consider the case $k=2$. Let $C_{1}$ and $C_{2}$ be the projections of $C$ to $X_{1}$ and $X_{2}$. If $x \in C_{1}$ and $y \in C_{2}$, there exist $u \in X_{1}$ and $v \in X_{2}$ such that the points $\bar{x}:=(x, v)$ and $\bar{y}:=(u, y)$ lie in $C$. It is immediate to observe that the point $(x, y)$ lies in $I(\bar{x}, \bar{y}) \subseteq C$. Finally, part 4 is Lemma 2.11 in Fio18.

Given $n \geq 2$, halfspaces $\mathfrak{h}_{1}, \ldots, \mathfrak{h}_{n}$ form a facing $n$-tuple if they are pairwise disjoint. We say that $\mathfrak{h}, \mathfrak{k} \in \mathscr{H}$ are strongly separated if $\overline{\mathfrak{h}} \cap \overline{\mathfrak{k}}=\emptyset$ and no $\mathfrak{j} \in \mathscr{H}$ is transverse to both $\mathfrak{h}$ and $\mathfrak{k}$. See Theorem 5.9 and Lemma 6.3 in Fio18 for the following.

Proposition 2.12. Let $X$ be an irreducible, complete, finite rank median space; let $\mathfrak{h}$ be a thick halfspace.

(1) If $X$ admits a Roller minimal action without wall inversions, there exist thick halfspaces $\mathfrak{h}^{\prime} \subseteq \mathfrak{h} \subseteq \mathfrak{h}^{\prime \prime}$ such that $\mathfrak{h}^{\prime}$ and $\mathfrak{h}^{\prime \prime *}$ are strongly separated.

(2) If $X$ admits a Roller nonelementary, Roller minimal action without walls inversions, $\mathfrak{h}$ is part of a facing $n$-tuple of thick halfspaces for every $n \geq 2$.

Every complete, finite rank median space can be isometrically embedded into its barycentric subdivision $X^{\prime}$. This is a complete median space of the same rank; see Section 2.3 in [Fio18]. When $X$ is the 0 -skeleton of a CAT(0) cube complex with the $\ell^{1}$ metric, the space $X^{\prime}$ is given by the 0 -skeleton of the customary barycentric subdivision.

We have a natural homomorphism Isom $X \hookrightarrow$ Isom $X^{\prime}$. Given any isometric action $\Gamma \curvearrowright X$, the induced action $\Gamma \curvearrowright X^{\prime}$ is without wall inversions. 
We write $\left(\mathscr{H}^{\prime}, \nu^{\prime}\right)$ instead of $\left(\mathscr{H}\left(X^{\prime}\right), \nu_{X^{\prime}}\right)$. There is an inclusion preserving map $p: \mathscr{H}^{\prime} \rightarrow \mathscr{H}$; it is surjective, (Isom $X$ )-equivariant and its fibres have cardinality at most two. We have $\#\left(p^{-1}(\mathfrak{h})\right)=2$ if and only if $\{\mathfrak{h}\}$ is an atom for $\widehat{\nu}$; in this case, we refer to each element of $p^{-1}(\mathfrak{h})$ as a hemiatom. The following is Lemma 5.7 in [Fio18.

Lemma 2.13. Let $X$ be a complete, finite rank median space. An action $\Gamma \curvearrowright X$ is Roller elementary if and only if the induced action $\Gamma \curvearrowright X^{\prime}$ is.

The sets $\{-1,1\}$ and $\{-1,0,1\}$ inherit a median algebra structure from the median space $\mathbb{R}$; in particular, we can consider the product median algebras $\{-1,1\}^{k}$ and $\{-1,0,1\}^{k}$ for every $k \geq 0$. For every point $x \in X^{\prime} \backslash X$, there exists a canonical, gate-convex subset $\widehat{C}(x) \subseteq X^{\prime}$; it is isomorphic to $\{-1,0,1\}^{k}$, for some $k \geq 1$, via an isomorphism that takes $x$ to the centre $(0, \ldots, 0)$. The intersection $C(x):=\widehat{C}(x) \cap X$ is gate-convex in $X$ and corresponds to the subset $\{-1,1\}^{k} \subseteq\{-1,0,1\}^{k}$. For $x \in X$, we set $\widehat{C}(x)=C(x)=\{x\}$. See Lemma 2.13 in [Fio18] for more details.

Lemma 2.14. Let $X$ be a complete, finite rank median space. Every infinite, convex subset $E \subseteq X^{\prime}$ intersects $X$.

Proof. Let $x \in E$ be a point minimising $R:=\operatorname{rank}(C(x))$; if $R=0$ we have $x \in E \cap X$. Otherwise, there exists a point $y \in E$ that does not lie in the finite set $\widehat{C}(x)$; the gate-projection $z$ of $y$ to $\widehat{C}(x)$ lies in $\widehat{C}(x) \backslash\{x\}$. In particular $C(z)$ is contained in a face of $C(x)$ and has strictly lower rank, a contradiction since $z \in I(x, y) \subseteq E$.

In particular, we obtain the following extension of Lemma 2.13.

Lemma 2.15. If $\Gamma \curvearrowright X$ is Roller nonelementary and Roller minimal, so is the action $\Gamma \curvearrowright X^{\prime}$.

Proof. Suppose for the sake of contradiction that $C \subseteq \overline{X^{\prime}}$ is a nonempty, $\Gamma$-invariant, closed, convex subset. By Corollary 4.31 in [Fio17] there exists a $\Gamma$-invariant component $W \subseteq \overline{X^{\prime}}$ with $W \cap C \neq \emptyset$. Note that $C \cap W$ is unbounded by Corollary 2.17 in [Fio18, since $\Gamma \curvearrowright X^{\prime}$ is Roller nonelementary by Lemma 2.13 . The component $W$ is the barycentric subdivision of a component $Z \subseteq \bar{X}$ and Lemma 2.14 implies that $C \cap Z \neq \emptyset$. Since $\Gamma \curvearrowright X$ is Roller minimal, we must have $Z=X$ and $C \cap X=X$; hence $X^{\prime} \subseteq C$ by part 2 of Proposition 2.15 in [Fio18]. We conclude that $C=\overline{X^{\prime}}$.

Let us now fix a basepoint $x \in X$, where $X$ is a complete finite rank median space; the following discussion is independent of our choice of $x$. A diverging chain of halfspaces is a sequence $\left(\mathfrak{h}_{n}\right)_{n \geq 0}$ such that $d\left(x, \mathfrak{h}_{n}\right) \rightarrow+\infty$ and $\mathfrak{h}_{n+1} \subseteq \mathfrak{h}_{n}$ for each $n \geq 0$; we use the same terminology for the set $\left\{\mathfrak{h}_{n} \mid n \geq 0\right\}$. The following notion will be key to our proof of Theorem A and to the discussion in Appendix A. It was first introduced by Hag13 in the context of $\operatorname{CAT}(0)$ cube complexes. 
Definition 2.16. Given $\xi \in \partial X$, a unidirectional boundary set (UBS) for $\xi$ is an inseparable subset $\Omega \subseteq \sigma_{\xi} \backslash \sigma_{x}$ containing a diverging chain of halfspaces.

Given UBS's $\Omega_{1}, \Omega_{2} \subseteq \sigma_{\xi} \backslash \sigma_{x}$, we say that $\Omega_{1}$ is almost contained in $\Omega_{2}$ if the halfspaces in $\Omega_{1} \backslash \Omega_{2}$ lie at uniformly bounded distance from $x$; this is denoted by $\Omega_{1} \preceq \Omega_{2}$. If $\Omega_{1} \preceq \Omega_{2}$ and $\Omega_{2} \preceq \Omega_{1}$, the UBS's are equivalent and we write $\Omega_{1} \sim \Omega_{2}$. We denote the equivalence class of $\Omega \subseteq \mathscr{H}$ by $[\Omega]$ and the set of all equivalence classes of UBS's for $\xi$ by $\overline{\mathcal{U}}(\xi)$; the relation $\preceq$ descends to a partial order on $\overline{\mathcal{U}}(\xi)$. A UBS $\Omega$ is said to be minimal if $[\Omega]$ is a minimal element of $(\overline{\mathcal{U}}(\xi), \preceq)$. A minimal UBS is equivalent to the inseparable closure of any diverging chain that it contains.

We define a directed graph $\mathcal{G}(\xi)$ as follows; also cf. [Hag17, Fio18]. The vertex set of $\mathcal{G}(\xi)$ is identified with the set of minimal elements of $(\overline{\mathcal{U}}(\xi), \preceq)$. Given diverging chains $\left(\mathfrak{h}_{m}\right)_{m \geq 0}$ and $\left(\mathfrak{k}_{n}\right)_{n \geq 0}$ in $\Omega_{1}$ and $\Omega_{2}$ respectively, we draw an oriented edge from $\left[\Omega_{1}\right]$ to $\left[\Omega_{2}\right]$ if almost every $\mathfrak{h}_{m}$ is transverse to almost every $\mathfrak{k}_{n}$, but not vice versa; this is independent of the choices involved. A subset $A \subseteq \mathcal{G}(\xi)^{(0)}$ is said to be inseparable if every directed path between vertices in $A$ only crosses vertices in $A$. For the following, see Lemma 4.5 and Proposition 4.7 in [Fio18.

Proposition 2.17. Let $X$ be a complete median space of finite rank $r$ and $\xi \in \partial X$.

(1) The graph $\mathcal{G}(\xi)$ has at most $r$ vertices and contains no directed cycles.

(2) The poset $(\overline{\mathcal{U}}(\xi), \preceq)$ is isomorphic to the poset of inseparable subsets of $\mathcal{G}(\xi)^{(0)}$, ordered by inclusion. The isomorphism maps $[\Omega] \in \overline{\mathcal{U}}(\xi)$ to the set of equivalence classes of minimal UBS's almost contained in $\Omega$. In particular, the set $\overline{\mathcal{U}}(\xi)$ is finite.

(3) Given a UBS $\Omega$ and a set $\left\{\Omega_{1}, \ldots, \Omega_{k}\right\}$ of representatives of all equivalence classes of minimal UBS's almost contained in $\Omega$, we have

$$
\sup _{\mathfrak{h} \in \Omega \triangle\left(\Omega_{1} \cup \ldots \cup \Omega_{k}\right)} d(x, \mathfrak{h})<+\infty .
$$

If $\Omega$ is a minimal UBS, we denote by $\Omega^{\prime}$ the subset of halfspaces $\mathfrak{k} \in \Omega$ that are not transverse to any diverging chain of halfspaces in $\Omega$. We say that $\Omega$ is reduced if $\Omega=\Omega^{\prime}$. We say that $\Omega$ is strongly reduced if we can write $\Omega=\mathcal{C}_{1} \sqcup \ldots \sqcup \mathcal{C}_{k}$ for some $k \geq 1$, where each $\mathcal{C}_{i}$ is totally ordered by inclusion and contains a diverging chain of halfspaces.

Consider the median spaces in Figures 1 and 2 , both are subsets of $\mathbb{R}^{2}$ with the restriction of the $\ell^{1}$ metric. In both cases, the UBS $\Omega=\sigma_{\xi} \backslash \sigma_{x}$ is minimal. Figure 1 shows that $\Omega$ can be reduced, but not strongly reduced. In Figure 2 the UBS $\Omega$ is strongly reduced and exhibits how the decomposition $\Omega=\mathcal{C}_{1} \sqcup \ldots \sqcup \mathcal{C}_{k}$ can require $k \geq 2$.

Lemma 2.18. Let $X$ be a complete, finite rank median space. Consider $x \in X$ and $\xi \in \partial X$; let $\Omega \subseteq \sigma_{\xi} \backslash \sigma_{x}$ be a minimal $U B S$.

(1) The subset $\Omega^{\prime}$ is a reduced UBS equivalent to $\Omega$. 


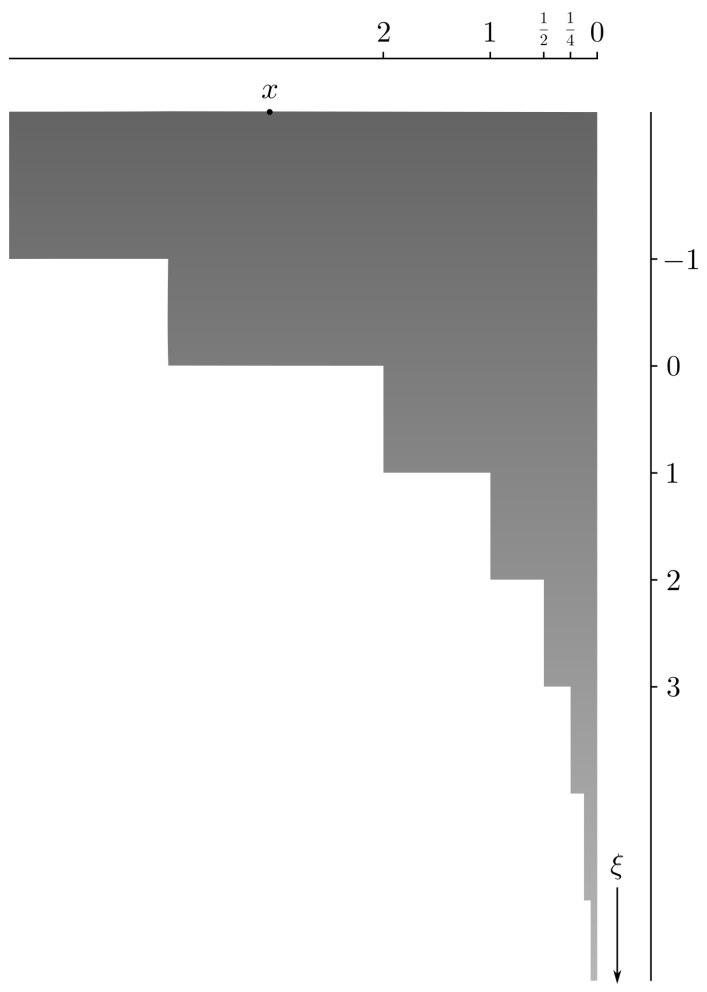

Figure 1

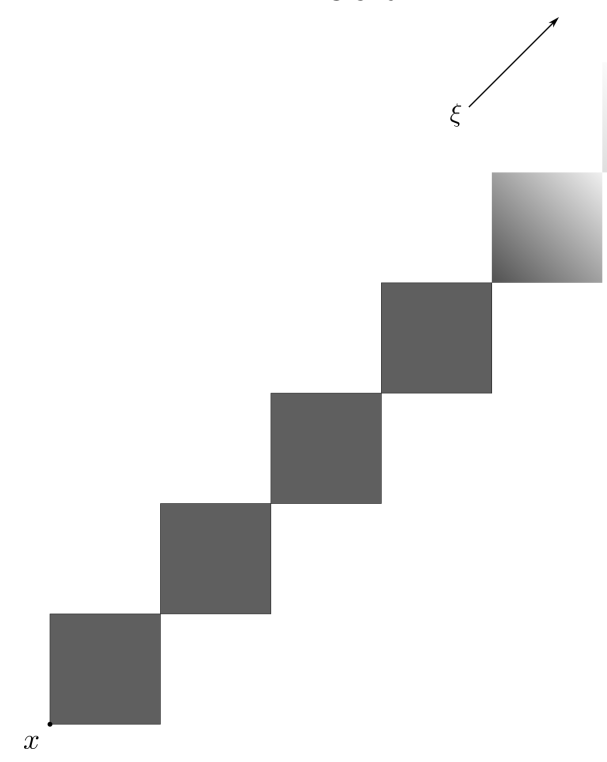

Figure 2 
(2) There exists a strongly reduced UBS contained in $\Omega$; all its sub-UBS's are strongly reduced.

(3) If $\Omega$ is strongly reduced, it is reduced.

Proof. If $\mathfrak{h} \subseteq \mathfrak{k}$ lie in $\sigma_{\xi} \backslash \sigma_{x}$ and $\mathfrak{k}$ is transverse to a diverging chain of halfspaces, then $\mathfrak{h}$ is transverse to an infinite subchain. This implies that $\Omega^{\prime}$ is inseparable; moreover, $\Omega \backslash \Omega^{\prime}$ cannot contain a diverging chain or $\Omega$ would contain two inequivalent UBS's. This proves part 1.

To obtain part 2, we decompose $\Omega=\mathcal{C}_{1} \sqcup \ldots \sqcup \mathcal{C}_{k}$ as in Lemma 2.2, let $A$ be the union of the sets $\mathcal{C}_{i}$ that do not contain a diverging chain. There exists $D<+\infty$ such that $d(x, \mathfrak{h}) \leq D$ for every $\mathfrak{h} \in A$. The set $\{\mathfrak{h} \in \Omega \mid d(x, \mathfrak{h})>D\}$ is a strongly reduced UBS and so are its sub-UBS's.

Regarding part 3 , decompose $\Omega=\mathcal{C}_{1} \sqcup \ldots \sqcup \mathcal{C}_{k}$, where each $\mathcal{C}_{i}$ is totally ordered by inclusion and contains a diverging chain. If there existed $\mathfrak{k} \in \Omega \backslash \Omega^{\prime}$, we would have $\mathfrak{k} \in \mathcal{C}_{i}$ for some $i$; in particular, $\mathfrak{k}$ would not be transverse to a diverging chain in $\mathcal{C}_{i}$. Since $\Omega$ is minimal, $\mathfrak{k}$ would not be transverse to any diverging chain in $\Omega$, a contradiction.

Given $\xi \in \partial X$, we denote by $\operatorname{Isom}_{\xi} X$ the group of isometries that fix $\xi$. Let $K_{\xi}$ be the kernel of the action of $\operatorname{Isom}_{\xi} X$ on $\overline{\mathcal{U}}(\xi)$; it is a finite-index subgroup of $\operatorname{Isom}_{\xi} X$.

If $\Omega \subseteq \sigma_{\xi} \backslash \sigma_{x}$ is a UBS, we denote by $K_{\Omega}$ the subgroup of $\operatorname{Isom}_{\xi} X$ that fixes the equivalence class $[\Omega]$. We can define a transfer character $\chi_{\Omega}: K_{\Omega} \rightarrow \mathbb{R}$ by the formula $\chi_{\Omega}(g)=\widehat{\nu}\left(g^{-1} \Omega \backslash \Omega\right)-\widehat{\nu}\left(\Omega \backslash g^{-1} \Omega\right)$; compare Section $4 . \mathrm{H}$ in Cor13]. This is a homomorphism and only depends on the equivalence class $[\Omega]$ (see Lemma 4.8 in [Fio18]). If $\Omega_{1}, \ldots, \Omega_{k}$ are a set of representatives for $\mathcal{G}(\xi)^{(0)}$, we can also consider the full transfer homomorphism $\chi_{\xi}=\left(\chi_{\Omega_{1}}, \ldots, \chi_{\Omega_{k}}\right): K_{\xi} \rightarrow \mathbb{R}^{k}$.

Proposition 2.19. Let $X$ be a complete, finite rank median space; consider $\xi \in \partial X$. The subgroup $K_{\xi}$ is open in Isom $_{\xi} X$ and the full transfer homomorphism $\chi_{\xi}: K_{\xi} \rightarrow \mathbb{R}^{k}$ is continuous. Every finitely generated subgroup of $\operatorname{ker} \chi_{\xi}$ has a finite orbit in $X$; if $X$ is connected, every finitely generated subgroup of ker $\chi_{\xi}$ fixes a point.

Before proving the proposition, we will need to obtain the following lemma. Note that, for every point $\xi \in \partial X$ and every halfspace $\mathfrak{h} \in \sigma_{\xi}$, the set $\mathscr{H}\left(\mathfrak{h}^{*} \mid \xi\right)=\left\{\mathfrak{k} \in \sigma_{\xi} \mid \mathfrak{k} \subseteq \mathfrak{h}\right\}$ is a UBS.

Lemma 2.20. For every thick halfspace $\mathfrak{h} \in \sigma_{\xi}$ and every $\epsilon>0$, there exists a neighbourhood $U$ of the identity in Isom $_{\xi} X$ such that $\mathscr{H}\left(\mathfrak{h}^{*} \mid \xi\right) \sim \mathscr{H}\left(g \mathfrak{h}^{*} \mid \xi\right)$ and $\widehat{\nu}\left(\mathscr{H}\left(\mathfrak{h}^{*} \mid \xi\right) \triangle \mathscr{H}\left(g \mathfrak{h}^{*} \mid \xi\right)\right)<\epsilon$ for all $g \in U$.

Proof. Pick a point $x \in X$ with $d(x, \mathfrak{h})>0$; in a neighbourhood of the identity of $\operatorname{Isom}_{\xi} X$, we have $x \in g \mathfrak{h}^{*}$. If $\mathfrak{k} \in \mathscr{H}\left(\mathfrak{h}^{*} \mid \xi\right) \backslash \mathscr{H}\left(g \mathfrak{h}^{*} \mid \xi\right)$ and $y$ is the gate-projection of $x$ to $\overline{\mathfrak{k}}$, we have $y \in g \overline{\mathfrak{h}}^{*}$ by part 4 of Proposition 2.3 . since $\overline{\mathfrak{k}} \cap g \overline{\mathfrak{h}}^{*} \neq \emptyset$ and $x \in g \overline{\mathfrak{h}^{*}}$; thus $d\left(y, g^{-1} y\right) \geq d\left(\mathfrak{k}, \mathfrak{h}^{*}\right)$. We conclude that, for every $\mathfrak{k} \in \mathscr{H}\left(\mathfrak{h}^{*} \mid \xi\right)$ with $d\left(\mathfrak{k}, \mathfrak{h}^{*}\right)>0$, there exists a neighbourhood $V_{\mathfrak{k}}$ of 
the identity in $\operatorname{Isom}_{\xi} X$ such that $\mathfrak{k} \in \mathscr{H}\left(g \mathfrak{h}^{*} \mid \xi\right)$ for all $g \in V_{\mathfrak{k}}$.

Decompose $\mathscr{H}\left(\mathfrak{h}^{*} \mid \xi\right)=\mathcal{C}_{1} \sqcup \ldots \sqcup \mathcal{C}_{k}$ as in Lemma 2.2. Let $\mathfrak{k}_{i}$ be the union of all $\mathfrak{k} \in \mathcal{C}_{i}$ with $d\left(\mathfrak{h}^{*}, \mathfrak{k}\right) \geq \frac{\epsilon}{2 k}$; if nonempty, it is a halfspace and $d\left(\mathfrak{h}^{*}, \mathfrak{k}_{i}\right) \geq \frac{\epsilon}{2 k}$. Elements of $\mathscr{H}\left(\mathfrak{h}^{*} \mid \xi\right)$ not contained in any $\mathfrak{k}_{i}$ form a subset of measure at most $\sum \widehat{\nu}\left(\mathscr{H}\left(\mathfrak{h}^{*} \mid \mathfrak{k}_{i}\right)\right) \leq \frac{\epsilon}{2}$. Let $V$ be the intersection of the $V_{\mathfrak{k}_{i}}$ for $\mathfrak{k}_{i} \neq \emptyset$; if $g \in V$, the set $\mathscr{H}\left(\mathfrak{h}^{*} \mid \xi\right) \backslash \mathscr{H}\left(g \mathfrak{h}^{*} \mid \xi\right)$ has measure at most $\frac{\epsilon}{2}$ and consists of halfspaces at uniformly bounded distance from $x \in X$. It now suffices to consider $U:=V \cap V^{-1}$.

Proof of Proposition 2.19. We only need to prove that $K_{\xi}$ is open and that $\chi_{\xi}$ is continuous; the rest of the statement is contained in Theorem $\mathrm{F}$ of [Fio18]. For every $v \in \mathcal{G}(\xi)^{(0)}$, there exists $\mathfrak{h}_{v}$ such that the vertices $w \in \mathcal{G}(\xi)^{(0)}$ with $w \preceq\left[\mathscr{H}\left(\mathfrak{h}_{v}^{*} \mid \xi\right)\right]$ are precisely $v$ and those that are at the other end of an incoming edge at $v$. Indeed, given a diverging chain in a UBS representing $v$, almost every halfspace in the chain can be chosen as $\mathfrak{h}_{v}$.

Let $A_{i}$ be the set of vertices $v \in \mathcal{G}(\xi)^{(0)}$ such that there exists no directed path of length $\geq i$ in $\mathcal{G}(\xi)$ that ends at $v$. Note that, by Proposition 2.17, we have $\emptyset=A_{0} \subsetneq A_{1} \subsetneq \ldots \subsetneq A_{k}=\mathcal{G}(\xi)^{(0)}$ for some $k \leq r$. We will show that the subgroup $K_{i} \leq \operatorname{Isom}_{\xi} X$ that fixes $A_{i} \subseteq \mathcal{G}(\xi)^{(0)}$ pointwise is open in $\operatorname{Isom}_{\xi} X$ and that, for every $[\Omega] \in A_{i}$, the homomorphism $\chi_{\Omega}: K_{i} \rightarrow \mathbb{R}$ is continuous. We proceed by induction on $i$, setting $K_{0}:=\operatorname{Isom}_{\xi} X$.

The base step is trivial. If $i \geq 1$, let $A_{i} \backslash A_{i-1}=\left\{v_{1}, \ldots, v_{s}\right\}$ and consider the halfspaces $\mathfrak{h}_{v_{1}}, \ldots, \mathfrak{h}_{v_{s}}$. Setting $\Xi_{j}:=\mathscr{H}\left(\mathfrak{h}_{v_{j}}^{*} \mid \xi\right)$, Lemma 2.20 provides a neighbourhood $U$ of id in $\operatorname{Isom}_{\xi} X$ such that $g \Xi_{j} \sim \Xi_{j}$ for all $j$ and all $g \in U$. A minimal UBS almost contained in $\Xi_{j}$ projects to an element of $A_{i-1}$ or to $v_{j}$; hence, we have $g v_{j}=v_{j}$ for every $g \in U \cap K_{i-1}$. Since, by the inductive hypothesis, $K_{i-1}$ is open, so is $K_{i}$. Continuity of the transfer characters is obtained with a similar argument.

2.2. Bridges. Let a median algebra $(M, m)$ and two gate-convex subsets $C_{1}$, $C_{2}$ be fixed throughout this section. All the following results have analogues in the context of CAT $(0)$ cube complexes; see Section 2.G in [CFI16.

Denote by $\pi_{i}: M \rightarrow C_{i}$ the gate-projection to $C_{i}$. We will refer to the sets

$$
\begin{aligned}
& S_{1}:=\left\{x_{1} \in C_{1} \mid \exists x_{2} \in C_{2} \text { s.t. }\left(x_{1}, x_{2}\right) \text { are gates for }\left(C_{1}, C_{2}\right)\right\}, \\
& S_{2}:=\left\{x_{2} \in C_{2} \mid \exists x_{1} \in C_{1} \text { s.t. }\left(x_{1}, x_{2}\right) \text { are gates for }\left(C_{1}, C_{2}\right)\right\},
\end{aligned}
$$

as the shores of $C_{1}$ and $C_{2}$, respectively. By part 3 of Proposition 2.3 , these coincide with $\pi_{1}\left(C_{2}\right)$ and $\pi_{2}\left(C_{1}\right)$, hence they are gate-convex. The map $\left.\pi_{2}\right|_{S_{1}}: S_{1} \rightarrow S_{2}$ is a bijection with inverse $\left.\pi_{1}\right|_{S_{2}} ;$ if $M$ arises from a median space $X$, this is an isometry as gate-projections are 1-Lipschitz. The bridge is the set

$$
B:=\bigsqcup_{x_{1} \in S_{1}} I\left(x_{1}, \pi_{2}\left(x_{1}\right)\right)=\bigsqcup_{x_{2} \in S_{2}} I\left(\pi_{1}\left(x_{2}\right), x_{2}\right) .
$$


The union is disjoint because, if $\left(x_{1}, x_{2}\right)$ is a pair of gates for $\left(C_{1}, C_{2}\right)$, we have $\pi_{i}\left(I\left(x_{1}, x_{2}\right)\right)=\left\{x_{i}\right\}$ for $i=1,2$; this follows from part 4 of Proposition 2.3 and the observation that $I\left(x_{1}, x_{2}\right) \cap C_{i}=\left\{x_{i}\right\}$.

Proposition 2.21. The bridge $B$ is gate-convex and

$$
\mathscr{W}(B)=\left(\mathscr{W}\left(C_{1}\right) \cap \mathscr{W}\left(C_{2}\right)\right) \sqcup \mathscr{W}\left(C_{1} \mid C_{2}\right) .
$$

For any pair of gates $\left(x_{1}, x_{2}\right)$, the bridge is canonically isomorphic to the product $S_{1} \times I\left(x_{1}, x_{2}\right)$; this is an isometry if $M$ arises from a median space.

Proof. Pick a pair of gates $\left(x_{1}, x_{2}\right)$, set $I:=I\left(x_{1}, x_{2}\right)$ and consider the morphism of median algebras $\phi:=\pi_{S_{1}} \times \pi_{I}: M \rightarrow S_{1} \times I$. If $\left(x_{1}^{\prime}, x_{2}^{\prime}\right)$ is another pair of gates, the projection $\pi_{I}$ provides an isomorphism $I\left(x_{1}^{\prime}, x_{2}^{\prime}\right) \rightarrow I$ mapping each $x_{i}^{\prime}$ to $x_{i}$. This observation and the decomposition $(*)$ above imply that the restriction $\left.\phi\right|_{B}$ is bijective.

The map $\pi_{B}=\left(\left.\phi\right|_{B}\right)^{-1} \circ \phi: M \rightarrow B$ is surjective and it is a gate-projection by Proposition 2.1 in [Fio17. By part 1 of Proposition 2.3 and the discussion above, every wall of $B$ arises either from a wall of $M$ cutting $S_{1}$ or from a wall of $M$ cutting $I$; the latter correspond to $\mathscr{W}\left(C_{1} \mid C_{2}\right)$ by part 2 of Proposition 2.3, so we are left to show that $\mathscr{W}\left(S_{1}\right)=\mathscr{W}\left(C_{1}\right) \cap \mathscr{W}\left(C_{2}\right)$. This follows from the fact that $S_{1}=\pi_{1}\left(C_{2}\right)$.

When $M$ arises from a median space $X$, the measure $\widehat{\nu}$ can be pushed forward via the standard two-to-one projection $\mathscr{H} \rightarrow \mathscr{W}$. For the purpose of the current proof, we denote by $\widehat{\mu}$ the resulting measure on $\mathscr{W}$. The fact that $\left.\phi\right|_{B}$ is an isometry then follows from the decomposition of $\mathscr{W}(B)$ above and the observation that $\frac{1}{2} \cdot \widehat{\mu}(\mathscr{W}(x \mid y))=d(x, y)$ for all $x, y \in X$.

We can extend the notion of strong separation to arbitrary gate-convex subsets of median algebras. We say that $C_{1}$ and $C_{2}$ are strongly separated if they are disjoint and $\mathscr{W}\left(C_{1}\right) \cap \mathscr{W}\left(C_{2}\right)=\emptyset$. Note that the condition $\mathscr{W}\left(C_{1}\right) \cap \mathscr{W}\left(C_{2}\right)=\emptyset$ alone already implies that $C_{1} \cap C_{2}$ consists of at most one point. In a median space, two halfspaces are strongly separated in the sense of Section 2.1 if and only if their closures are strongly separated according to the definition above; see Lemma 2.23 below for a stronger result.

Proposition 2.21 implies that two disjoint, gate-convex sets are strongly separated if and only if their shores are singletons; this yields the following result.

Corollary 2.22. Let $C_{1}, C_{2} \subseteq M$ be strongly separated. There exists a unique pair of gates $\left(x_{1}, x_{2}\right)$ and $\pi_{1}\left(C_{2}\right)=\left\{x_{1}\right\}, \pi_{2}\left(C_{1}\right)=\left\{x_{2}\right\}$.

We will also need the following:

Lemma 2.23. Let $\mathfrak{h}, \mathfrak{k} \in \mathscr{H}$ be strongly separated as halfspaces. The closures $H, K$ of $\widetilde{\mathfrak{h}}, \widetilde{\mathfrak{k}}$ in $\bar{X}$ are strongly separated as subsets of $\bar{X}$.

Proof. Since $\mathfrak{h}, \mathfrak{k}$ have disjoint closures in $X$, the sets $H$ and $K$ are disjoint by Helly's Theorem. By Proposition 2.21, it then suffices to prove that the shore $S \subseteq H$ is a singleton. Suppose for the sake of contradiction that $S$ 
contains distinct points $\xi, \eta$ and let $\xi^{\prime}, \eta^{\prime} \in K$ be their projections to $K$; in particular, $\sigma_{\eta} \backslash \sigma_{\xi}=\sigma_{\eta^{\prime}} \backslash \sigma_{\xi^{\prime}}$. Given $\mathfrak{j} \in \sigma_{\eta} \backslash \sigma_{\xi}$, the argument at the beginning of the proof shows that the closures of $\mathfrak{j}$ and $\mathfrak{h}$ inside $X$ intersect nontrivially; by Lemma 3.6 in [Fio17, almost every $\mathfrak{j} \in \sigma_{\eta} \backslash \sigma_{\xi}$ intersects $\mathfrak{h}$. Considering complements, we conclude that almost every $\mathfrak{j} \in \sigma_{\eta} \backslash \sigma_{\xi}$ is transverse to $\mathfrak{h}$ and, similarly, to $\mathfrak{k}$. Since $d(\xi, \eta)>0$, there exists such a $\mathfrak{j}$, contradicting the fact that $\mathfrak{h}, \mathfrak{k}$ are strongly separated.

2.3. The Haagerup class. Let $X$ be a median space, $G$ a topological group and $p \in[1,+\infty)$. Given a Banach space $E$, we denote by $\mathscr{U}(E)$ the group of linear isometries of $E$.

An isometric action $G \curvearrowright X$ corresponds to a measure preserving action $G \curvearrowright(\mathscr{H}, \widehat{\nu})$. We obtain a continuous representation $\rho_{p}: G \rightarrow \mathscr{U}\left(L^{p}(\mathscr{H}, \widehat{\nu})\right)$; when $p=2$, we simply write $\rho:=\rho_{2}$. We will use interchangeably the notations $H_{c}^{1}\left(G, \rho_{p}\right)$ and $H_{c}^{1}\left(G, L^{p}(\mathscr{H}, \widehat{\nu})\right)$ to denote continuous cohomology.

Given $x \in X$, we can consider the continuous 1-cocycle $b^{x}: G \rightarrow L^{p}(\mathscr{H}, \widehat{\nu})$ defined by $b^{x}(g):=g \cdot \mathbb{1}_{\sigma_{x}}-\mathbb{1}_{\sigma_{x}} ;$ it satisfies $\left\|b^{x}(g)\right\|_{p}=(2 \cdot d(x, g x))^{1 / p}$. We will refer to $b^{x}$ as a Haagerup cocycle. The cohomology class $\left[b^{x}\right] \in H_{c}^{1}\left(G, \rho_{p}\right)$ does not depend on the point $x$ and we will simply denote it by $[b]$.

The action $G \curvearrowright X$ has bounded orbits if and only if the affine action $G \curvearrowright L^{p}(\mathscr{H}, \widehat{\nu})$ induced by $b^{x}$ fixes a point; this follows for instance from the Ryll-Nardzewski Theorem for $p>1$ and from Theorem A in [BGM12] in the case $p=1$. Thus, we have $[b]=0$ if and only if the action $G \curvearrowright X$ has bounded orbits.

We can also consider the projection $\overline{[b]}$ of $[b]$ to reduced continuous cohomology, which carries more interesting geometrical information (see Theorem $\mathrm{A}$ in the introduction). We will refer to $\overline{[b]} \in \overline{H_{c}^{1}}(G, \rho)$ as the Haagerup class of $G \curvearrowright X$. The choice of $p=2$ here is not particularly relevant and the same discussion could be equally carried out for any other $p \in[1,+\infty)$ with few complications; see Remark 3.6 .

We conclude this section by collecting a few straightforward lemmata for later use. Let $\mathcal{H}$ be a Hilbert space and $G \rightarrow \mathscr{U}(\mathcal{H})$ a continuous unitary representation.

Lemma 2.24. If $H \leq G$ is open and finite-index, functoriality induces an injective map $\overline{H_{c}^{1}}(G, \overline{\mathcal{H}}) \hookrightarrow \overline{H_{c}^{1}}(H, \mathcal{H})$.

Lemma 2.25. Given a $G$-invariant decomposition $\mathcal{H}=\mathcal{H}_{1} \perp \mathcal{H}_{2}$, the projections onto the two factors induce $\overline{H_{c}^{1}}(G, \mathcal{H}) \simeq \overline{H_{c}^{1}}\left(G, \mathcal{H}_{1}\right) \oplus \overline{H_{c}^{1}}\left(G, \mathcal{H}_{2}\right)$.

Recall that we denote by $X^{\prime}$ the barycentric subdivision of $X$ and by $i: X \hookrightarrow X^{\prime}$ the standard inclusion; we write $\left(\mathscr{H}^{\prime}, \nu^{\prime}\right)$ instead of $\left(\mathscr{H}\left(X^{\prime}\right), \widehat{\nu}_{X^{\prime}}\right)$. Every isometric action $G \curvearrowright X$ also induces a continuous representation $\rho^{\prime}: G \rightarrow \mathscr{U}\left(L^{2}\left(\mathscr{H}^{\prime}, \nu^{\prime}\right)\right)$.

Lemma 2.26. Let $X$ be complete and finite rank and let $G \curvearrowright X$ be an isometric action. The projection $p: \mathscr{H}^{\prime} \rightarrow \mathscr{H}$ induces an isometric embedding 
$p^{*}: L^{2}(\mathscr{H}, \widehat{\nu}) \hookrightarrow L^{2}\left(\mathscr{H}^{\prime}, \nu^{\prime}\right)$ and a monomorphism

$$
p_{*}: \overline{H_{c}^{1}}(G, \rho) \hookrightarrow \overline{H_{c}^{1}}\left(G, \rho^{\prime}\right)
$$

taking the Haagerup class of $G \curvearrowright X$ to the Haagerup class of $G \curvearrowright X^{\prime}$.

Proof. The fact that $p^{*}$ is an isometric embedding follows from the observation that $p_{*} \nu^{\prime}=\widehat{\nu}$. Injectivity of $p_{*}$ follows from Lemma 2.25 applied to $L^{2}(\mathscr{H}, \widehat{\nu})$ and its orthogonal complement. Finally, if $x \in X$ and $b^{x}$ is the corresponding Haagerup cocycle for $G \curvearrowright X$, the cocycle $p^{*} \circ b^{x}$ is the Haagerup cocycle for $G \curvearrowright X^{\prime}$ relative to the point $i(x) \in X^{\prime}$.

\section{HAAGERUP ClASS AND ElEMENTARITy OF ACTIONS.}

3.1. The main statement. Let $X$ be a complete, finite rank median space and let $G \curvearrowright X$ be an isometric action of a topological group $G$. The goal of this section is to prove Theorem A.

By Lemmata 2.13 and 2.26, it suffices to consider the case when $G \curvearrowright X$ is without wall inversions; this will be a standing assumption throughout the rest of the section.

Lemma 3.1. Suppose that $X$ is irreducible and that $G \curvearrowright X$ is Roller minimal and Roller nonelementary. There exists a non-abelian free subgroup $H \leq G$ such that $\rho$ has no $H$-almost-invariant vectors and $H \curvearrowright X$ has unbounded orbits.

Proof. Part 2 of Proposition 6.4 in Fio18 provides a non-abelian free subgroup $H \leq G$ and a measurable, $*$-invariant partition $\mathscr{H}=\bigsqcup_{h \in H} \mathscr{H}_{h}$ with $g \mathscr{H}_{h}=\mathscr{H}_{g h}$ for all $g, h \in H$. It is immediate from the construction of $H$ that it acts on $X$ with unbounded orbits. If there existed a sequence of almost invariant vectors $\left(F_{n}\right)_{n \geq 0}$ in $L^{2}(\mathscr{H}, \widehat{\nu})$, say with $\left\|F_{n}\right\|_{2}=1$, we could define functions $f_{n} \in \ell^{2}(H)$ by $f_{n}(h):=\left\|F_{n} \mathbb{1}_{\mathscr{H}_{h}}\right\|_{2}$. It is immediate to check that $\left\|f_{n}\right\|_{2}=1$ for every $n \geq 0$ and, for every $g \in H$,

$$
\begin{aligned}
\left\|g f_{n}-f_{n}\right\|_{2}^{2} & =\sum_{h \in H}\left(\left\|F_{n} \mathbb{1}_{\mathscr{H}_{g^{-1}}}\right\|_{2}-\left\|F_{n} \mathbb{1}_{\mathscr{H}_{h}}\right\|_{2}\right)^{2} \\
& =\sum_{h \in H}\left(\left\|\left(g F_{n}\right) \mathbb{1}_{\mathscr{H}_{h}}\right\|_{2}-\left\|F_{n} \mathbb{1}_{\mathscr{H}_{h}}\right\|_{2}\right)^{2} \\
& \leq \sum_{h \in H}\left\|\left(g F_{n}\right) \mathbb{1}_{\mathscr{H}_{h}}-F_{n} \mathbb{1}_{\mathscr{H}_{h}}\right\|_{2}^{2}=\left\|g F_{n}-F_{n}\right\|_{2}^{2} \underset{n \rightarrow+\infty}{\longrightarrow} 0 .
\end{aligned}
$$

Thus, the regular representation of $H$ would contain almost-invariant vectors, implying amenability of $H$ (see e.g. Theorem G.3.2 in [BdlHV08]). This is a contradiction.

We can already prove the "only if" half of Theorem A.

Proposition 3.2. If $G \curvearrowright X$ is Roller nonelementary, we have $\overline{[b]} \neq 0$. 
Proof. Note that, by functoriality of reduced cohomology, it suffices to consider the case when $G$ has the discrete topology; thus, we do not need to worry about continuity issues. We proceed by induction on $r=\operatorname{rank}(X)$. When $r=0$, all actions are Roller elementary; suppose for the rest of the proof that $r \geq 1$.

We can assume that $G \curvearrowright X$ is Roller minimal. Indeed, if $C \subseteq Z \subseteq \bar{X}$ are the subsets provided by Proposition 2.9. the action $G \curvearrowright C$ is again without wall inversions and $\operatorname{rank}(C) \leq r$, by Proposition 2.7. The $G$-equivariant, measurable partition $\mathscr{H}=\mathscr{H}_{C} \sqcup\left(\sigma_{C} \cup \sigma_{C}^{*}\right)$ induces an orthogonal decomposition of $L^{2}(\mathscr{H}, \widehat{\nu})$ and a $G$-equivariant splitting

$$
\overline{H_{c}^{1}}\left(G, L^{2}(\mathscr{H}, \widehat{\nu})\right)=\overline{H_{c}^{1}}\left(G, L^{2}\left(\mathscr{H}(C), \widehat{\nu}_{C}\right)\right) \oplus \overline{H_{c}^{1}}\left(G, L^{2}\left(\sigma_{C} \cup \sigma_{C}^{*}, \widehat{\nu}\right)\right) .
$$

If $p_{1}$ and $p_{2}$ are the orthogonal projections of $L^{2}(\mathscr{H}, \widehat{\nu})$ onto the two direct summands, we can write $\overline{\left[b^{x}\right]}=\overline{\left[p_{1} b^{x}\right]}+\overline{\left[p_{2} b^{x}\right]}$ for every $x \in X$. Note that the gate-projection $\pi_{C}: \bar{X} \rightarrow \bar{C}$ maps $x$ to a point $\xi \in C$. The cocycle $p_{1} b^{x}$ is precisely the Haagerup cocycle $b^{\xi}$ for the action $G \curvearrowright C$, by part 2 of Proposition 2.7. In particular, if $\overline{\left[b^{\xi}\right]} \neq 0$, we can conclude that $\overline{[b]} \neq 0$.

We thus assume in the rest of the proof that $X=C$, i.e. that $G \curvearrowright X$ is Roller minimal. If $X$ is irreducible, Lemma 3.1 provides $i: H \hookrightarrow G$ such that $H \curvearrowright X$ has unbounded orbits and $\rho$ has no $H$-almost-invariant vectors. The first condition implies that $i^{*}[b] \neq 0$; the second condition and Guichardet's trick (Theorem 1 in Gui72]) thus yield $i^{*}[b] \neq 0$. In particular, $\overline{[b]} \neq 0$.

If instead $X$ splits as a nontrivial product $X_{1} \times X_{2}$, there exists a finiteindex subgroup $j: G_{0} \hookrightarrow G$ preserving this decomposition (see part 2 of Proposition 2.11). It suffices to show that $j^{*} \overline{[b]} \neq 0$. Writing $\widehat{\nu}_{i}$ instead of $\widehat{\nu}_{X_{i}}$, Proposition 2.11 and Lemma 2.25 imply:

$\overline{H_{c}^{1}}\left(G_{0}, L^{2}(\mathscr{H}, \widehat{\nu})\right)=\overline{H_{c}^{1}}\left(G_{0}, L^{2}\left(\mathscr{H}\left(X_{1}\right), \widehat{\nu}_{1}\right)\right) \oplus \overline{H_{c}^{1}}\left(G_{0}, L^{2}\left(\mathscr{H}\left(X_{2}\right), \widehat{\nu}_{2}\right)\right)$;

hence, if $x=\left(x_{1}, x_{2}\right)$, we have $\overline{\left[b^{x}\right]}=\overline{\left[b_{X_{1}}^{x_{1}}\right]}+\overline{\left[b_{X_{2}}^{x_{2}}\right]}$. The action $G_{0} \curvearrowright X$ is Roller nonelementary, since $G_{0}$ is finite-index in $G$; thus, up to exchanging the two factors, $G_{0} \curvearrowright X_{1}$ is Roller nonelementary. Since $\operatorname{rank}\left(X_{1}\right)<r$, the inductive hypothesis guarantees that $\overline{\left[b_{X_{1}}^{x_{1}}\right]} \neq 0$ and this concludes the proof.

Before proving the rest of Theorem $\mathrm{A}$ we need to obtain a few more results.

Lemma 3.3. If the $G$-orbit of $\xi \in \bar{X}$ is finite, the $G$-stabiliser of $\xi$ is open.

Proof. Suppose that $G \xi=\{\xi\} \sqcup\left\{\xi_{1}, \ldots, \xi_{k}\right\}$ and $d\left(\xi, \xi_{i}\right) \geq \epsilon>0$ for all $i$, where $d$ is the extended metric on $\bar{X}$. By Proposition 4.24 in [Fio17], we can find $x_{i}, y_{i} \in X$ such that, denoting by $\pi_{i}$ the projection to $I_{i}:=I\left(x_{i}, y_{i}\right)$, we have $d\left(\pi_{i}(\xi), \pi_{i}\left(\xi_{i}\right)\right)>\frac{3}{4} \epsilon$. In a neighbourhood $U \subseteq G$ of the identity element we have $d\left(g x_{i}, x_{i}\right)<\frac{1}{4} \epsilon, d\left(g y_{i}, y_{i}\right)<\frac{1}{4} \epsilon$ and $d\left(g \pi_{i}(\xi), \pi_{i}(\xi)\right)<\frac{1}{4} \epsilon$, for all $i$.

If $g \in U$, we have $d\left(\pi_{g I_{i}}\left(\xi_{i}\right), \pi_{i}\left(\xi_{i}\right)\right) \leq d\left(g x_{i}, x_{i}\right)+d\left(g y_{i}, y_{i}\right)<\frac{1}{2} \epsilon$; if in addition we had $g \xi=\xi_{i}$, we would have $\pi_{g I_{i}}\left(\xi_{i}\right)=g \pi_{i}(\xi)$. As a consequence, 
$d\left(\pi_{i}(\xi), \pi_{i}\left(\xi_{i}\right)\right) \leq d\left(\pi_{i}(\xi), g \pi_{i}(\xi)\right)+d\left(g \pi_{i}(\xi), \pi_{i}\left(\xi_{i}\right)\right)<\frac{3}{4} \epsilon$, which would contradict our choice of $I_{i}$. We conclude that $U$ is contained in the stabiliser of $\xi$, which must be open in $G$.

The proof of the following fact is rather lengthy and technical; it will be carried out in Appendix A. See Definition 2.16 for the definition of UBS.

Proposition 3.4. Let $\xi \in \partial X$ and $K \subseteq$ Isom $_{\xi} X$ be a compact set of isometries acting trivially on $\overline{\mathcal{U}}(\xi)$. There exists a point $x_{K} \in X$ such that $\sigma_{\xi} \backslash \sigma_{x_{K}}$ coincides, up to a null set, with $\Omega_{K}:=\Omega_{K}^{1} \sqcup \ldots \sqcup \Omega_{K}^{k}$, where

- each $\Omega_{K}^{i}$ is a strongly reduced, minimal UBS;

- if $g \in K$ we have $g \Omega_{K}^{i} \subseteq \Omega_{K}^{i}$ whenever $\chi_{\Omega_{K}^{i}}(g) \geq 0$ and $g \Omega_{K}^{i} \supseteq \Omega_{K}^{i}$ whenever $\chi_{\Omega_{K}^{i}}(g) \leq 0$;

- if $i \neq j$ and $g \in K$, we have $\Omega_{K}^{i} \cap g \Omega_{K}^{j}=\emptyset$.

Given points $\xi \in \partial X, x \in X$ and a UBS $\Omega \subseteq \sigma_{\xi} \backslash \sigma_{x}$, we can define a function $\alpha_{\Omega}: \Omega \rightarrow \mathbb{R}$ as $\alpha_{\Omega}(\mathfrak{h}):=\widehat{\nu}(\mathscr{H}(x \mid \mathfrak{h}) \cap \Omega)$. The dependence on the point $x$ is not particularly relevant, so we do not record it in our notation. We can consider the sets $\Omega_{c}:=\left\{\mathfrak{h} \in \Omega \mid \alpha_{\Omega}(\mathfrak{h}) \leq c\right\}$. In Appendix A we will also obtain the following result (see Lemma A.9).

Proposition 3.5. Suppose that $\Omega$ is minimal and strongly reduced. Let $K \subseteq$ Isom $_{\xi} X$ be a compact set of isometries such that $g \Omega \subseteq \Omega$ for all $g \in K$. As $c \rightarrow+\infty$, the functions

$$
(g-i d) \cdot\left[-\left(1-\frac{\alpha_{\Omega}}{c}\right) \cdot \mathbb{1}_{\Omega_{c}}\right]
$$

converge to $\mathbb{1}_{\Omega \backslash g \Omega}$ in $L^{2}(\mathscr{H}, \widehat{\nu})$, uniformly in $g \in K$. If instead $g \Omega \supseteq \Omega$ for all $g \in K$, they converge to the function $-\mathbb{1}_{g \Omega \backslash \Omega}$.

We are finally ready to complete the proof of Theorem A.

Proof of Theorem A. By Proposition 3.2, it suffices to consider the case when $G$ has a finite orbit in $\bar{X}$ and, by Lemmata 2.24 and 3.3 , we can actually assume that $G$ fixes a point $\xi \in \bar{X}$. If $\xi \in X$, we have $[b]=0$; suppose instead that $\xi \in \partial X$. By Proposition 2.19, an open, finite-index subgroup $G_{0} \leq G$ acts trivially on $\overline{\mathcal{U}}(\xi)$; by Lemma 2.24 , it suffices to consider the case $G=G_{0}$.

Fix $x \in X$. For every $\epsilon>0$ and every compact subset $K \subseteq G$, we need to construct a function $\psi \in L^{2}(\mathscr{H}, \widehat{\nu})$ such that $\left\|b^{x}(g)-(g \psi-\psi)\right\|_{2}<\epsilon$ for all $g \in K$. Considering the point $x_{K} \in X$ provided by Proposition 3.4, it suffices to find a function $\phi \in L^{2}(\mathscr{H}, \widehat{\nu})$ such that $\left\|b^{x_{K}}(g)-(g \phi-\phi)\right\|_{2}<\epsilon$ for all $g \in K$ and then set $\psi:=\phi+\mathbb{1}_{\sigma_{x}}-\mathbb{1}_{\sigma_{x_{K}}}$. If $g \in K$, considering all equalities up to null sets, we have:

$$
\begin{aligned}
\sigma_{g x_{K}} \backslash \sigma_{x_{K}} & =\left[\left(\sigma_{g x_{K}} \backslash \sigma_{x_{K}}\right) \cap \sigma_{\xi}\right] \sqcup\left[\left(\sigma_{g x_{K}} \backslash \sigma_{x_{K}}\right) \cap \sigma_{\xi}^{*}\right] \\
& =\left[\left(\sigma_{\xi} \backslash \sigma_{x_{K}}\right) \backslash\left(\sigma_{\xi} \backslash \sigma_{g x_{K}}\right)\right] \sqcup\left[\left(\sigma_{\xi} \backslash \sigma_{g x_{K}}\right) \backslash\left(\sigma_{\xi} \backslash \sigma_{x_{K}}\right)\right]^{*} \\
& =\left(\Omega_{K} \backslash g \Omega_{K}\right) \sqcup\left(g \Omega_{K} \backslash \Omega_{K}\right)^{*} .
\end{aligned}
$$


In particular, since by construction $\Omega_{K}^{i} \cap g \Omega_{K}^{j}=\emptyset$ whenever $i \neq j$,

$$
\sigma_{g x_{K}} \backslash \sigma_{x_{K}}=\bigsqcup_{i=1}^{k}\left(\Omega_{K}^{i} \backslash g \Omega_{K}^{i}\right) \sqcup\left(g \Omega_{K}^{i} \backslash \Omega_{K}^{i}\right)^{*} .
$$

Introducing the notation $\mathbb{2}_{A}:=\mathbb{1}_{A}-\mathbb{1}_{A^{*}}$ for subsets $A \subseteq \mathscr{H}$, we can rewrite:

$$
b^{x_{K}}(g)=\mathcal{2}_{\sigma_{g x} \backslash} \backslash \sigma_{x_{K}}=\sum_{\chi_{\Omega_{K}^{i}(g)>0}} \mathcal{2}_{\Omega_{K}^{i} \backslash g \Omega_{K}^{i}}-\sum_{\chi_{\Omega_{K}^{i}(g)<0}} \mathcal{L}_{g \Omega_{K}^{i} \backslash \Omega_{K}^{i}} .
$$

For $c>0$, consider the function:

$$
G_{c}:=\sum_{i=1}^{k}-\left(1-\frac{\alpha_{\Omega_{K}^{i}}}{c}\right) \mathcal{P}_{\left(\Omega_{K}^{i}\right)_{c}} .
$$

Proposition 3.5 shows that it suffices to take $\phi=G_{c}$ for large $c$.

Remark 3.6. Theorem A also holds for the analogous class in $\overline{H_{c}^{1}}\left(G, \rho_{p}\right)$, for every $p \in[1,+\infty)$. Indeed, Lemma 2.25 applies to any decomposition of a Banach space into closed subspaces. In the proof of Lemma 2.26, a closed complement to $L^{p}(\mathscr{H}, \widehat{\nu})$ within $L^{p}\left(\mathscr{H}^{\prime}, \nu^{\prime}\right)$ is always provided by the subspace of functions on $\mathscr{H}^{\prime}$ that take opposite values on hemiatoms. Theorem 1 in Gui72 holds for representations into general Banach spaces (although this does not appear in Gui72]). Finally, if $F$ is a non-abelian free group, $\ell^{p}(F)$ has no $F$-almost-invariant vectors for every $p \in[1,+\infty)$.

The value of $p$ also has little importance for most of the material in Appendix A. Note however that Proposition 3.5 fails for $p=1$; one needs to consider functions with a quicker decay in that case.

3.2. Elementarity and Shalom's property $H_{F D}$. Let $X$ be a complete, finite rank median space and let $G$ be a topological group acting on $X$ by isometries. Here we prove Theorem $\mathrm{G}$ and Corollaries $\mathrm{H}$ and $\mathrm{I}$.

We will need the following ergodicity result for the action $G \curvearrowright \mathscr{H}$.

Lemma 3.7. Suppose that $X$ is irreducible and let $G \curvearrowright X$ be a Roller nonelementary, Roller minimal action. Let $E \subseteq \mathscr{H}$ be a measurable subset such that $\widehat{\nu}(g E \triangle E)=0$ for all $g \in G$. Then $\widehat{\nu}(E)$ is either 0 or $+\infty$.

Proof. Without loss of generality we can assume that $G \curvearrowright X$ is without wall inversions, as Lemma 2.15 allows us to pass to the barycentric subdivision $X^{\prime}$ if necessary. Now, suppose that $E$ is such a set and $0<\widehat{\nu}(E)<+\infty$. Since $X$ has finite rank, we can find a thick halfspace $\mathfrak{h}$ such that, replacing $E$ with $E^{*}$ if necessary, the set $E_{\mathfrak{h}}:=E \cap\{\mathfrak{k} \in \mathscr{H} \mid \mathfrak{k} \subseteq \mathfrak{h}\}$ satisfies $a:=\widehat{\nu}\left(E_{\mathfrak{h}}\right)>0$. By part 2 of Proposition 2.12, the halfspace $\mathfrak{h}$ is part of a facing $n$-tuple with $n>\frac{1}{a} \cdot \widehat{\nu}(E)$. By Proposition 2.10, there exist $g_{2}, \ldots, g_{n} \in G$ such that $\mathfrak{h}, g_{2} \mathfrak{h}, \ldots, g_{n} \mathfrak{h}$ are a facing $n$-tuple. The sets $E_{\mathfrak{h}}, g_{2} E_{\mathfrak{h}}, \ldots, g_{n} E_{\mathfrak{h}}$ are pairwise disjoint and contained in $E$, up to null sets. However, their union has measure $n a>\widehat{\nu}(E)$, a contradiction. 
Proof of Theorem G. Suppose for the sake of contradiction that $G \curvearrowright X$ is Roller nonelementary. Without loss of generality, we can assume that $X$ has minimal rank $r$ among complete median spaces admitting Roller nonelementary actions of $G$. In particular, $X$ must be irreducible, see the proof of Proposition 3.2. By Proposition 2.9, we can also assume that $G \curvearrowright X$ is Roller minimal. Theorem A guarantees that $\overline{H_{c}^{1}}(G, \rho) \neq\{0\}$ and, since $G$ has property $H_{F D}$, there exists a finite dimensional subrepresentation $V<L^{2}(\mathscr{H}, \widehat{\nu})$. We will construct a measurable $G$-invariant subset of $\mathscr{H}$ with positive, finite measure, thus violating Lemma 3.7.

Let $f_{1}, \ldots, f_{k}$ be measurable functions on $\mathscr{H}$ whose equivalence classes in $L^{2}(\mathscr{H}, \widehat{\nu})$ form an orthonormal basis of $V$. Define, for $c>0$,

$E_{c}:=\left\{\mathfrak{h} \in \mathscr{H} \mid \exists \alpha=\left(\alpha_{1}, \ldots, \alpha_{k}\right) \in \mathbb{S}^{k-1}\right.$ s.t. $\left.\left|\left(\alpha_{1} f_{1}+\ldots+\alpha_{k} f_{k}\right)(\mathfrak{h})\right|>c\right\}$.

Since in the definition of $E_{c}$ it suffices to look at $\alpha$ 's lying in a countable dense subset of $\mathbb{S}^{k-1}$, we conclude that $E_{c}$ is measurable. If $\mathfrak{h} \in E_{c}$, we must have $\left|f_{i}(\mathfrak{h})\right|>\frac{c}{k}$ for some $i$, hence $\widehat{\nu}\left(E_{c}\right)<+\infty$; if $c$ is sufficiently small, we have $\widehat{\nu}\left(E_{c}\right)>0$. Given $g \in G$, there exist real numbers $\alpha_{i j}, 1 \leq i, j \leq k$, such that, outside a measure zero set, we have $f_{i}=\sum_{j} \alpha_{i j}\left(g f_{j}\right)$ for every $i$. If $\mathfrak{h} \in E_{c} \backslash g E_{c}$, we must have $f_{i}(\mathfrak{h}) \neq \sum_{j} \alpha_{i j}\left(g f_{j}\right)(\mathfrak{h})$ for some $i$; we conclude that $\widehat{\nu}\left(g E_{c} \triangle E_{c}\right)=0$ for all $g \in G$.

Proof of Corollary $H$. Cocompactness of the action implies that $X$ is finite dimensional. By Proposition 2.19 and Theorem G. there exists a finiteindex subgroup $\Gamma_{0} \leq \Gamma$ and a normal subgroup $N \triangleleft \Gamma_{0}$ consisting of elliptic elements, such that $\Gamma_{0} / N$ is abelian. Since $\Gamma$ acts freely, $N$ is trivial.

Recall that, in Gromov's density model, random groups at density $d<\frac{1}{2}$ are nonelementary hyperbolic with overwhelming probability Gro93, Oll04]. Together with Theorem 10.4 in OW11, Corollary H] then immediately implies Corollary 1 .

\section{SUPERRIGIDITY.}

4.1. The superrigidity result. Let $X$ be a complete median space of finite rank $r$ and $\Gamma \curvearrowright X$ an action by isometries of a discrete group $\Gamma$.

Lemma 4.1. Suppose $\mathfrak{h}_{1}, \mathfrak{h}_{2}, \mathfrak{h}_{3} \in \mathscr{H}$ form a facing triple; let $\mathfrak{k}_{i} \in \mathscr{H}$ and $\mathfrak{h}_{i}^{*}$ be strongly separated for $i=1,2,3$. There exists a point $z \in X$ such that $m\left(\xi_{1}, \xi_{2}, \xi_{3}\right)=z$ whenever $\xi_{i} \in \widetilde{\mathfrak{k}}_{i}$.

Proof. Let $C$ be the intersection of the closures of $\widetilde{\mathfrak{h}}_{i}^{*}$ inside $\bar{X}$; it is nonempty, closed and convex. Given points $\xi_{i} \in \widetilde{\mathfrak{k}}_{i}$, set $m:=m\left(\xi_{1}, \xi_{2}, \xi_{3}\right)$. By convexity we have $I\left(\xi_{2}, \xi_{3}\right) \subseteq \widetilde{\mathfrak{h}}_{1}^{*}$, hence $m \in \widetilde{\mathfrak{h}}_{1}^{*}$; permuting the indices, we obtain $m \in C$. In particular, denoting by $\pi_{C}$ the gate projection $\bar{X} \rightarrow C$, we have $m=\pi_{C} m\left(\xi_{1}, \xi_{2}, \xi_{3}\right)=m\left(\pi_{C} \xi_{1}, \pi_{C} \xi_{2}, \pi_{C} \xi_{3}\right)$.

The closures of $\widetilde{\mathfrak{h}}_{i}^{*}$ and $\widetilde{\mathfrak{k}}_{i}$ in $\bar{X}$ are strongly separated by Lemma 2.23 . let $\left\{x_{i}\right\}$ be the shore of $\overline{\mathfrak{h}_{i}^{*}}$ and set $z:=m\left(x_{1}, x_{2}, x_{3}\right)$. By Corollary 2.22 . 
we have $\pi_{C}\left(\xi_{i}\right)=x_{i}$, hence $m=z$ no matter which points $\xi_{i} \in \widetilde{\mathfrak{k}}_{i}$ we have chosen.

In addition to Theorem $\mathrm{A}$ and Shalom's superrigidity [Sha00, the following is a key ingredient in the proof of Theorem B. Since $X$ lacks a cellular structure in general, we are forced to concoct a more elaborate proof for Lemma 4.2, if compared to analogues in the context of trees (pp.44-45 in [Sha00]) and cube complexes (Claim 6.1 in [CFI16]).

Lemma 4.2. Suppose that $X$ is irreducible and assume that $\Gamma \curvearrowright X$ is Roller nonelementary and Roller minimal. Given $0 \neq f \in L^{2}(\mathscr{H}, \widehat{\nu})$, consider

$$
\mathscr{S}(f):=\left\{\left(g_{n}\right) \in \Gamma^{\mathbb{N}} \mid g_{n} g \cdot f \underset{n \rightarrow+\infty}{\longrightarrow} g \cdot f, \forall g \in \Gamma\right\} .
$$

There exists $z \in X$ such that, for every $\left(g_{n}\right) \in \mathscr{S}(f)$, there exists $N \geq 0$ such that $g_{n} \cdot z=z$ for all $n \geq N$.

Proof. By part 1 of Proposition 2.11, the barycentric subdivision $X^{\prime}$ of $X$ is an irreducible median space of the same rank. The action $\Gamma \curvearrowright X^{\prime}$ is without wall inversions, Roller nonelementary and Roller minimal by Lemma 2.15 . As usual, we write $\left(\mathscr{H}^{\prime}, \nu^{\prime}\right)$ for $\left(\mathscr{H}\left(X^{\prime}\right), \widehat{\nu}_{X^{\prime}}\right)$.

The function $f \in L^{2}(\mathscr{H}, \widehat{\nu})$ induces $f^{\prime} \in L^{2}\left(\mathscr{H}^{\prime}, \nu^{\prime}\right)$ with $\mathscr{S}(f) \subseteq \mathscr{S}\left(f^{\prime}\right)$. We approximate $f^{\prime}$ by a linear combination $F$ of characteristic functions of halfspace intervals $\mathscr{H}^{\prime}\left(x_{1} \mid y_{1}\right), \ldots, \mathscr{H}^{\prime}\left(x_{k} \mid y_{k}\right)$ such that $\left\|F-f^{\prime}\right\|<\frac{1}{3}\left\|f^{\prime}\right\|$.

Proposition 2.10 implies that $\nu^{\prime}\left(\mathscr{H}^{\prime}\right)=+\infty$; all halfspace intervals have finite measure, so there exists $\mathfrak{h}^{\prime} \in \mathscr{H}^{\prime}$ such that $x_{i}, y_{i} \in \mathfrak{h}^{\prime}$ for all $i$. Propositions 2.10 and 2.12 provide a thick halfspace $\mathfrak{h} \in \mathscr{H}^{\prime}$ such that $\mathfrak{h}^{*}$ and $\mathfrak{h}^{\prime}$ are strongly separated; in particular, $\mathfrak{h}$ contains every wall in the set $\mathscr{W}^{\prime}\left(x_{1} \mid y_{1}\right) \cup \ldots \cup \mathscr{W}^{\prime}\left(x_{k} \mid y_{k}\right)$.

Propositions 2.10 and 2.12 also provide $\gamma_{1} \in \Gamma$ such that $\mathfrak{h}$ and $\gamma_{1} \mathfrak{h}$ are strongly separated and $\gamma_{2} \in \Gamma$ such that $\gamma_{1} \mathfrak{h}^{*}$ and $\gamma_{2} \mathfrak{h}$ are strongly separated. We can assume without loss of generality that $d\left(\gamma_{1} \mathfrak{h}^{*}, \gamma_{2} \mathfrak{h}\right) \geq 1$, as by Proposition 2.1 the quantity $d\left(\gamma_{1} \mathfrak{h}^{*}, \gamma_{2}^{n} \mathfrak{h}\right)$ diverges as $n$ goes to infinity. Thus, we can choose elements $\gamma_{m} \in \Gamma$ such that $\mathfrak{h}^{*} \supsetneq \gamma_{1} \mathfrak{h} \supsetneq \gamma_{2} \mathfrak{h} \supsetneq \ldots$ and, for all $i \geq 1$, the halfspaces $\gamma_{i} \mathfrak{h}^{*}$ and $\gamma_{i+1} \mathfrak{h}$ are strongly separated and at distance at least 1 .

We denote by $\mathcal{S} \subseteq \mathscr{H}^{\prime}$ the support of the function $F$ and by $\mathcal{P}$ the set of all points $x_{i}, y_{i}$. Let $D$ be the maximum distance from $\mathfrak{h}^{*}$ of a point of $\mathcal{P}$ and $d:=\lceil D+2\rceil$. Let us fix an integer $m>d$ and $g \in \Gamma$ such that $\left\|g \gamma_{m} f^{\prime}-\gamma_{m} f^{\prime}\right\|<\frac{1}{3}\left\|f^{\prime}\right\|$ and $\left\|g f^{\prime}-f^{\prime}\right\|<\frac{1}{3}\left\|f^{\prime}\right\|$. We prove that $g \gamma_{m} \mathfrak{h} \subseteq \gamma_{m-d} \mathfrak{h}$.

A straightforward repeated application of the triangle inequality yields $\|g F-F\|<2\|F\|$ and $\left\|g \gamma_{m} F-\gamma_{m} F\right\|<2\|F\| ;$ thus, $\widehat{\nu}(\mathcal{S} \cap g \mathcal{S})>0$ and $\widehat{\nu}\left(\gamma_{m} \mathcal{S} \cap g \gamma_{m} \mathcal{S}\right)>0$. Let $\mathfrak{w}$ be a wall corresponding to a halfspace in $\gamma_{m} \mathcal{S} \cap g \gamma_{m} \mathcal{S}$. Since $\mathfrak{w}$ is contained in both $\gamma_{m} \mathfrak{h}$ and $g \gamma_{m} \mathfrak{h}$ and $\Gamma \curvearrowright X^{\prime}$ is without wall inversions, we conclude that $\gamma_{m} \mathfrak{h} \cap g \gamma_{m} \mathfrak{h} \neq \emptyset$. A similar argument shows that $\mathfrak{h} \cap g \mathfrak{h} \neq \emptyset$. 
Let $\mathfrak{u}$ be the wall corresponding to $g \gamma_{m} \mathfrak{h}$. If $\mathfrak{u}$ is contained in $\gamma_{m-1} \mathfrak{h}$, we either have $g \gamma_{m} \mathfrak{h} \subseteq \gamma_{m-1} \mathfrak{h}$ or $\gamma_{m-1} \mathfrak{h}^{*} \cap g \gamma_{m} \mathfrak{h}^{*}=\emptyset$. The former case immediately yields $g \gamma_{m} \mathfrak{h} \subseteq \gamma_{m-d} \mathfrak{h}$, while the latter leads to a contradiction as $\mathfrak{h} \subseteq \gamma_{m-1} \mathfrak{h}^{*}$ and $g \mathfrak{h} \subseteq g \gamma_{m} \mathfrak{h}^{*}$ intersect.

If instead $\mathfrak{u}$ is not contained in $\gamma_{m-1} \mathfrak{h}$, it is contained in $\gamma_{m} \mathfrak{h}^{*}$, by strong separation. Let $1 \leq l \leq m$ be minimum such that $\gamma_{l} \mathfrak{h}^{*}$ contains $\mathfrak{u}$. We have $\gamma_{l} \mathfrak{h} \subseteq g \gamma_{m} \mathfrak{h}$, since $\gamma_{l} \mathfrak{h} \cap g \gamma_{m} \mathfrak{h} \supseteq \gamma_{m} \mathfrak{h} \cap g \gamma_{m} \mathfrak{h} \neq \emptyset$.

Let $\mathfrak{k}$ be the side of $\mathfrak{w}$ that is contained in $\gamma_{m} \mathfrak{h}$. Since either $\mathfrak{k}$ or $\mathfrak{k}^{*}$ lies in $g \gamma_{m} \mathcal{S}$, there exists $q \in \mathcal{P}$ such that $g \gamma_{m} q \in \mathfrak{k} \subseteq \gamma_{m} \mathfrak{h}$. Hence,

$$
m-l \leq d\left(\gamma_{m} \mathfrak{h}, \gamma_{l} \mathfrak{h}^{*}\right) \leq d\left(g \gamma_{m} q, g \gamma_{m} \mathfrak{h}^{*}\right)=d\left(q, \mathfrak{h}^{*}\right) \leq D
$$

and $m-l+2 \leq D+2 \leq d$. By strong separation and minimality of $l$, the wall $\mathfrak{u}$ is contained in $\gamma_{l-2} \mathfrak{h} \subseteq \gamma_{m-d} \mathfrak{h}$. Hence $g \gamma_{m} \mathfrak{h} \subseteq \gamma_{m-d} \mathfrak{h}$, since otherwise $g \gamma_{m} \mathfrak{h}^{*} \cap \gamma_{m-d} \mathfrak{h}^{*}=\emptyset$ would again violate the fact that $\mathfrak{h} \cap g \mathfrak{h} \neq \emptyset$.

Now consider the intersection $C$ of the closures in $\overline{X^{\prime}}$ of the halfspaces $\gamma_{m} \widetilde{\mathfrak{h}}$. It consists of a single point $\xi$ since any $\mathfrak{j} \in \mathscr{H}^{\prime}$ with $\widetilde{\mathfrak{j}} \cap C \neq \emptyset$ and $\tilde{\mathfrak{j}}^{*} \cap C \neq \emptyset$ would have to be transverse to almost all $\gamma_{m} \mathfrak{h}$, violating strong separation. Strong separation also implies that $\xi$ actually lies in $\bar{X} \subseteq \overline{X^{\prime}}$.

Given $\left(g_{n}\right) \in \mathscr{S}\left(f^{\prime}\right)$ we can assume, removing a finite number of elements if necessary, that $\left\|g_{n} f^{\prime}-f^{\prime}\right\|<\frac{1}{3}\left\|f^{\prime}\right\|$ for all $n$. Let $N(m)$ be a natural number such that $\left\|g_{n} \gamma_{m} f^{\prime}-\gamma_{m} f^{\prime}\right\|<\frac{1}{3}\left\|f^{\prime}\right\|$ for all $n \geq N(m)$. When $n \geq N(m)$, we have shown that $g_{n} \gamma_{m} \mathfrak{h} \subseteq \gamma_{m-d} \mathfrak{h}$; thus we have $g_{n} \xi \in g_{n} \gamma_{m} \widetilde{\mathfrak{h}} \subseteq \gamma_{m-d} \widetilde{\mathfrak{h}}$. In this case, strong separation implies that $\sigma_{g_{n} \xi} \triangle \sigma_{\xi}$ consists only of halfspaces whose corresponding walls are contained in $\gamma_{m-d-1} \mathfrak{h}$. This shows that $\limsup \sigma_{g_{n} \xi} \triangle \sigma_{\xi}=\emptyset$; we conclude that $g_{n} \xi \rightarrow \xi$ in the topology of $\bar{X}$, for every $\left(g_{n}\right) \in \mathscr{S}\left(f^{\prime}\right)$.

We finally construct the point $z \in X$. Let $\mathfrak{j}, \mathfrak{m}$ be thick halfspaces of $X^{\prime}$ so that $\mathfrak{m}^{*}$ and $\mathfrak{j}$ are strongly separated and $\xi \in \widetilde{\mathfrak{j}}$. Part 2 of Proposition 2.12 provides a facing triple consisting of $\mathfrak{m}, \mathfrak{m}_{1}, \mathfrak{m}_{2}$. We choose thick halfspaces $\mathfrak{j}_{1}, \mathfrak{j}_{2} \in \mathscr{H}^{\prime}$ such that $\mathfrak{m}_{i}^{*}$ and $\mathfrak{j}_{i}$ are strongly separated for $i=1,2$; by Proposition 2.10 we can find $h_{i} \in \Gamma$ such that $h_{i} \mathfrak{j} \subseteq \mathfrak{j}_{i}$. Let $z \in X^{\prime}$ be the point provided by Lemma 4.1 applied to $\mathfrak{j}, \mathfrak{j}_{1}, \mathfrak{j}_{2}$ and $\mathfrak{m}, \mathfrak{m}_{1}, \mathfrak{m}_{2}$; in particular, we have $z=m\left(\xi, h_{1} \xi, h_{2} \xi\right)$, hence $z \in X$.

Since the set $\mathscr{S}\left(f^{\prime}\right)$ is closed under conjugation by elements of $\Gamma$, we have $g_{n} h_{i} \xi \rightarrow h_{i} \xi$ for all $\left(g_{n}\right) \in \mathscr{S}\left(f^{\prime}\right)$. Hence, given $\left(g_{n}\right) \in \mathscr{S}\left(f^{\prime}\right)$, there exists $N \geq 0$ such that for every $n \geq N$ we have $g_{n} \xi \in \widetilde{\mathfrak{j}}$ and $g_{n} h_{i} \xi \in \widetilde{\mathfrak{j}}_{i}$, for $i=1,2$. Thus $g_{n} z=g_{n} m\left(\xi, h_{1} \xi, h_{2} \xi\right)=m\left(g_{n} \xi, g_{n} h_{1} \xi, g_{n} h_{2} \xi\right)=z$.

In the rest of the section, we consider a locally compact group $G$ and a lattice $\Gamma<G$. Any Borel fundamental domain $U \subseteq G$ defines a cocycle $\alpha: G \times U \rightarrow \Gamma$ such that $g u \in \alpha(g, u) \cdot U$. We say that $\Gamma$ is square-integrable if $\Gamma$ is finitely generated and $U$ can be chosen so that

$$
\int_{U}|\alpha(g, u)|_{S}^{2} d u<+\infty, \forall g \in G
$$


here $d u$ is the Haar measure on $U$ and $|\cdot|_{S}$ denotes the word length with respect to a finite generating set $S \subseteq \Gamma$. Integrability does not depend on the choice of $S$. Uniform lattices are always square-integrable and a few nonuniform examples were mentioned in the introduction; see Sha00, Rém99, Rém05, CR09, CR10 for more details and examples.

We assume that $G$ splits as a product $G_{1} \times \ldots \times G_{\ell}$, where each $G_{i}$ is compactly generated and $\ell \geq 2$. We also require the lattice $\Gamma<G$ to be irreducible, i.e. to project densely into each factor $G_{i}$.

Consider a unitary representation $\pi: \Gamma \rightarrow \mathscr{U}(\mathcal{H})$; we denote by $\mathcal{H}^{0}$ the subspace of invariant vectors. Let $c: \Gamma \rightarrow \mathcal{H}$ be a 1-cocycle for $\pi$. We will make use of the following result of $Y$. Shalom in an essential way; see page 14 and Theorem 4.1 in [Sha00] for a proof.

Theorem 4.3. Suppose that $\Gamma$ is square-integrable and that $\mathcal{H}^{0}=\{0\}$. There exist $\Gamma$-invariant closed subspaces $\mathcal{H}_{i} \leq \mathcal{H}, i=1, \ldots, \ell$, where the restriction $\pi_{i}: \Gamma \rightarrow \mathscr{U}\left(\mathcal{H}_{i}\right)$ extends to a continuous representation $\overline{\pi_{i}}: G \rightarrow \mathscr{U}\left(\mathcal{H}_{i}\right)$ that factors through the projection $p_{i}: G \rightarrow G_{i}$. Furthermore, there are cocycles $c_{i}: \Gamma \rightarrow \mathcal{H}_{i}, i=1, \ldots, \ell$, such that $c$ and $c_{1}+\ldots+c_{\ell}$ represent the same class in $\overline{H^{1}}(\Gamma, \pi)$.

The following is a version of our Theorem $\mathrm{B}$ under stronger hypotheses.

Theorem 4.4. Suppose that $\Gamma$ is square-integrable and $X$ is irreducible; let $\Gamma \curvearrowright X$ be Roller nonelementary and Roller minimal. There exists a $\Gamma$-invariant, closed median subalgebra $Y \subseteq X$ where $\Gamma \curvearrowright Y$ extends to a continuous action $G \curvearrowright Y$. Moreover, $G \curvearrowright Y$ factors through a projection $p_{i}: G \rightarrow G_{i}$

Proof. We have $\overline{H^{1}}(\Gamma, \rho) \neq\{0\}$ by Theorem A. Lemma 3.7 implies that $\rho$ has no nonzero invariant vectors; thus, Theorem 4.3 provides a $\Gamma$-invariant subspace $\{0\} \neq \mathcal{H}_{i} \subseteq L^{2}(\mathscr{H}, \widehat{\nu})$ where the action of $\Gamma$ extends to a continuous action of $G$ factoring through a projection $p_{i}: G \rightarrow G_{i}$.

Pick any $0 \neq f \in \mathcal{H}_{i}$ and consider the set $\mathscr{S}(f)$ introduced in Lemma 4.2 . Any sequence $\left(g_{n}\right) \in \Gamma^{\mathbb{N}}$ with $p_{i}\left(g_{n}\right) \rightarrow$ id lies in $\mathscr{S}(f)$. Thus, Lemma 4.2 implies that the $\Gamma$-invariant set

$$
Y:=\left\{x \in X \mid \forall\left(g_{n}\right) \in \Gamma^{\mathbb{N}} \text { s.t. } p_{i}\left(g_{n}\right) \rightarrow \mathrm{id} \text {, we have } g_{n} x \rightarrow x\right\}
$$

is nonempty. Note that $Y$ is a median subalgebra of $X$, thus the restriction of the metric of $X$ gives $Y$ a structure of median space. Since $Y$ is a closed subset of $X$, it is a complete median space. Finally, Proposition 4.3 in [Sha00] provides a continuous extension to $G$ of $\Gamma \curvearrowright Y$ and this factors through the projection $p_{i}$.

The assumption that $\Gamma \curvearrowright X$ be Roller minimal and Roller nonelementary can be replaced with the (stronger) requirement that $\Gamma$ have no finite orbit in the visual boundary of the $\operatorname{CAT}(0)$ space $\widehat{X}$; see Proposition 5.2 in [Fio18]. 
The homomorphism $\phi: G \rightarrow$ Isom Y provided by Theorem 4.4 is continuous with respect to the topology of pointwise convergence. We remark however that $\phi$ remains continuous even if we endow Isom $Y$ with the topology mentioned in Remark 4.5 below; this will be a key point in our proof of Theorem C.

Remark 4.5. In the proof of Theorem 4.4, Lemma 4.2 actually yields that the smaller set

$$
Y_{0}:=\left\{x \in X \mid \forall\left(g_{n}\right) \in \Gamma^{\mathbb{N}} \text { s.t. } p_{i}\left(g_{n}\right) \rightarrow \mathrm{id}, \exists N \geq 0 \text { s.t. } g_{n} x=x, \forall n \geq N\right\}
$$

is nonempty. Thus, $\phi: G \rightarrow$ Isom $\mathrm{Y}$ is continuous with respect to the topology on Isom $Y$ that is generated by stabilisers of points of $Y_{0}$. In the statement of Theorem 4.4, we can always take $Y$ to be the closure of $Y_{0}$ in $X$.

This topology on Isom $Y$ might seem a lot finer than the topology of pointwise convergence. To clarify this phenomenon, we mention the following fact, without proof. Let $W$ be an irreducible, complete, finite rank median space admitting a Roller nonelementary, Roller minimal action; then there exists a dense, convex subset $C \subseteq W$ such that, for every $x \in C$, the stabiliser of $x$ is open for the topology of pointwise convergence on Isom $W$. It is not hard to derive this from Lemma 4.1.

Relaxing the hypotheses of Theorem 4.4, we obtain Theorem B for all square-integrable lattices:

Corollary 4.6. Suppose that $\Gamma$ is square-integrable; let $\Gamma \curvearrowright X$ be Roller nonelementary. There exist a finite index subgroup $\Gamma_{0} \leq \Gamma, a \Gamma_{0}$-invariant component $Z \subseteq \bar{X}$ and a $\Gamma_{0}$-invariant closed median subalgebra $Y \subseteq Z$ where the action $\Gamma_{0} \curvearrowright Y$ extends to a continuous action $G_{0} \curvearrowright Y$, for an open finite index subgroup $G_{0} \leq G$.

Proof. We proceed by induction on $\operatorname{rank}(X)$; when the rank is zero there is nothing to prove, so we assume that the statement holds for all median spaces of rank at most $r-1$. By Proposition 2.9, there exists a $\Gamma$-invariant, closed, convex subset $D$ of a component $W \subseteq \bar{X}$ such that $\Gamma \curvearrowright D$ is Roller minimal and Roller nonelementary. If $W \subseteq \partial X$, we have $\operatorname{rank}(D)<r$ and we conclude by the inductive hypothesis; thus we can assume that $W=X$.

Let $D=D_{1} \times \ldots \times D_{k}$ be the splitting of $D$ into irreducible factors provided by Proposition 2.11. If $k=1$, the result follows from Theorem 4.4. If $k \geq 2$, let $\Gamma_{1} \leq \Gamma$ be a finite index subgroup preserving the splitting of $D$; up to permuting the factors, we can assume that $\Gamma_{1} \curvearrowright D_{i}$ is Roller nonelementary for $1 \leq i \leq s$ and Roller elementary for $i>s$. A further finite index subgroup $\Gamma_{2} \leq \Gamma_{1}$ fixes a point $\xi_{i} \in \overline{D_{i}}$ for each $i>s$; we denote by $Z_{i} \subseteq \overline{D_{i}}$ the component containing $\xi_{i}$. Note that $\Gamma_{2}$ is a square-integrable, irreducible lattice in an open, finite index subgroup of $G$.

Since $\operatorname{rank}\left(D_{i}\right)<r$, for each $i \leq s$ the inductive hypothesis yields a finite index subgroup $\Gamma_{0 i} \leq \Gamma_{2}$, an open finite index subgroup $G_{0 i} \leq G$ and a $\Gamma_{0 i}$-invariant, closed median subalgebra $Y_{i}$ of a component $Z_{i} \subseteq \overline{D_{i}}$ where 
the action of $\Gamma_{0 i}$ extends to a continuous action of $G_{0 i}$. Let $\Gamma_{0}$ be the intersection of all $\Gamma_{0 i}$ and $G_{0}$ be the intersection of all $G_{0 i}$, for $i \leq s$. The set $Y:=Y_{1} \times \ldots \times Y_{s} \times\left\{\xi_{s+1}\right\} \times \ldots \times\left\{\xi_{k}\right\} \subseteq \bar{D}$ is a closed median subalgebra of $Z_{1} \times \ldots \times Z_{k}$, which is a component of $\bar{D}$; in particular, $Z_{1} \times \ldots \times Z_{k}$ is a closed, convex subset of a component $Z \subseteq \bar{X}$. The action $\Gamma_{0} \curvearrowright Y$ trivially extends to a continuous action $G_{0} \curvearrowright Y$.

We now describe two examples that illustrate how:

- in Theorem 4.4 the space $Y$ cannot be taken to coincide with $X$, nor with a convex subset (Example 4.7);

- in Corollary 4.6 it cannot be avoided to pass to the finite index subgroup $\Gamma_{0}$, even when the action is Roller minimal (Example 4.8).

The actions that we consider are actually on CAT(0) square complexes. Since Burger-Mozes groups play an important role in the construction of the two examples, we briefly recall a few facts regarding their construction.

Given an integer $n \geq 3$, we denote by $T_{n}$ the $n$-regular tree and by $A_{n}$ the group of even permutations on $n$ elements. We fix a legal colouring on $T_{n}$, i.e. a way of associating an integer in $\{1, \ldots, n\}$ to every edge of $T_{n}$ so that we see all $n$ integers around each vertex; in particular, we have a bijection $i_{v}: \operatorname{lk}(v) \rightarrow\{1, \ldots, n\}$ for every vertex $v$. Let $U\left(A_{n}\right) \leq$ Isom $T_{n}$ be the subgroup of isometries $g$ such that $i_{g v} \circ g \circ i_{v}^{-1} \in A_{n}$ for every vertex $v$ of $T_{n}$; we denote by $U\left(A_{n}\right)^{+}$the intersection of $U\left(A_{n}\right)$ with the subgroup of Isom $T_{n}$ generated by edge stabilisers. If $n \geq 4$, the subgroup $U\left(A_{n}\right)^{+}$has index 2 in $U\left(A_{n}\right)$, see Proposition 3.2.1 in BM00a.

The subgroup $U\left(A_{n}\right)$ is closed in Isom $T_{n}$; in particular, it is locally compact, second countable and compactly generated (e.g. by Theorem 4.C.5 and Proposition 5.B.5 in [CdlH16]). By Theorem 6.3 in [BM00b], there exists a uniform irreducible lattice $\Lambda \leq U\left(A_{2 k}\right) \times U\left(A_{2 k}\right)$ for every integer $k \geq 19$.

For the next two examples, we fix such a lattice $\Lambda$. Let $p_{1}, p_{2}: \Lambda \rightarrow U\left(A_{2 k}\right)$ be the projections into the two factors and set $\Lambda_{0}:=p_{1}^{-1}\left(U\left(A_{2 k}\right)^{+}\right)$; this is an irreducible lattice in the open, index 2 subgroup $U\left(A_{2 k}\right)^{+} \times U\left(A_{2 k}\right)$ of $U\left(A_{2 k}\right) \times U\left(A_{2 k}\right)$. Let $\tau: \Lambda \rightarrow \mathbb{Z} / 2 \mathbb{Z}$ be the homomorphism with kernel $\Lambda_{0}$.

Example 4.7. Given any tree $T$, we can blow up every edge to a square as in Figure 3, thus obtaining a "tree of squares" T. Adjacent squares only share a vertex; if $T$ has no leaves, each square has a pair of opposite vertices that are shared with other squares and a pair of opposite vertices that are not shared. The space $\mathbb{T}$ is a complete, rank two median space in which $T$ embeds as a median subalgebra; edges of $T$ correspond to diagonals joining shared pairs of vertices of squares of $\mathrm{T}$.

We can embed Isom $T \hookrightarrow$ Isom $\mathbb{T}$ by extending each isometry of $T$ so that the restriction to each square is orientation preserving. Let $\sigma \in \mathrm{Isom} \mathrm{T}$ be the isometry that fixes pointwise the image of the embedding $T \hookrightarrow \mathbb{T}$ and acts on each square as a reflection in the diagonal; we have $\sigma^{2}=$ id and Isom $T \times\langle\sigma\rangle \hookrightarrow$ Isom $\mathbb{T}$. 


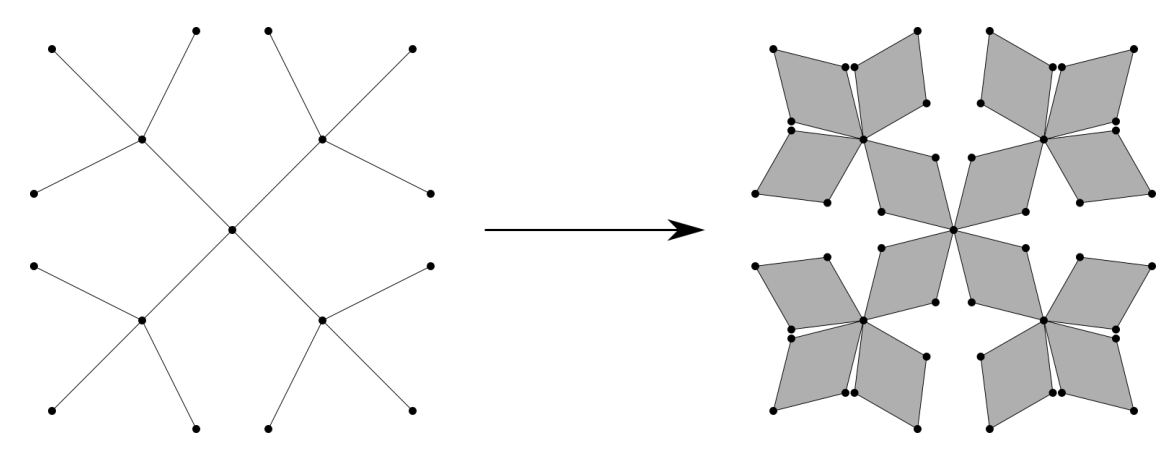

Figure 3

Let us now apply this construction to $T=T_{2 k}$, writing $\mathbb{T}=\mathbb{T}_{2 k}$. Let $\Lambda \leq U\left(A_{2 k}\right) \times U\left(A_{2 k}\right)$ be as above. Viewing $p_{2}: \Lambda \rightarrow U\left(A_{2 k}\right)$ as a homomorphism into Isom $T_{2 k}$ we can define a homomorphism $\Lambda \rightarrow$ Isom $T_{2 k} \times\langle\sigma\rangle$ by $\lambda \mapsto\left(p_{2}(\lambda), \tau(\lambda)\right)$. We denote by $\psi$ the composition of this map with the embedding Isom $T_{2 k} \times\langle\sigma\rangle \hookrightarrow$ Isom $\mathbb{T}_{2 k}$.

The action $\Lambda \curvearrowright \mathbb{T}_{2 k}$ induced by $\psi$ is Roller nonelementary and Roller minimal since the action $\Lambda \curvearrowright T_{2 k}$ induced by $p_{2}$ is. As $\mathbb{T}_{2 k}$ is irreducible, Theorem 4.4 guarantees a continuous extension of $\Lambda \curvearrowright Y$ to $U\left(A_{2 k}\right)$, for some $\Lambda$-invariant median subalgebra of $\mathrm{T}_{2 k}$. Indeed, one can take $Y$ to be the image of $T_{2 k} \hookrightarrow \mathbb{T}_{2 k}$.

However, $Y$ cannot be taken to be a convex subspace (or even a subcomplex) of $\mathbb{T}_{2 k}$. Indeed, $Y$ would be forced to be the whole $\mathbb{T}_{2 k}$, as this is the only $\Lambda$-invariant convex subset of $\mathbb{T}_{2 k}$. The action $\Lambda \curvearrowright \mathbb{T}_{2 k}$ does not extend to $U\left(A_{2 k}\right) \times U\left(A_{2 k}\right)$ by factoring via $p_{1}$; this is because, whenever elements $g_{n} \in \Lambda$ satisfy $p_{1}\left(g_{n}\right) \rightarrow \mathrm{id}$, the sequence $\left(p_{2}\left(g_{n}\right)\right)$ must diverge. However, $\Lambda \curvearrowright \mathbb{T}_{2 k}$ also does not extend by factoring through $p_{2}$ : we have $\overline{p_{2}\left(\Lambda_{0}\right)}=\overline{p_{2}(\Lambda)}=U\left(A_{2 k}\right)$, but $\psi\left(\Lambda_{0}\right)$ is contained in the closed subgroup Isom $T_{2 k}<$ Isom $\mathbb{T}_{2 k}$ and $\psi(\Lambda)$ is not.

In the next example we maintain the notation introduced before Example 4.7 .

Example 4.8. Choose an element $g \in \Lambda \backslash \Lambda_{0}$ and consider the action $\Lambda_{0} \curvearrowright T_{2 k} \times T_{2 k}$ given by $\lambda \cdot(x, y)=\left(p_{2}(\lambda) \cdot x, p_{2}\left(g^{-1} \lambda g\right) \cdot y\right)$. Since the action $U\left(A_{2 k}\right) \curvearrowright T_{2 k}$ does not preserve any proper closed subtree, the same holds for the action of $p_{2}\left(\Lambda_{0}\right)$. Part 3 of Proposition 2.11 then implies that $\Lambda_{0}$ does not leave any proper, closed, convex subset of $T_{2 k} \times T_{2 k}$ invariant. Note that no component of $\partial\left(T_{2 k} \times T_{2 k}\right)=\left(\partial T_{2 k} \times \overline{T_{2 k}}\right) \cup\left(\overline{T_{2 k}} \times \partial T_{2 k}\right)$ is preserved by $\Lambda_{0}$, as this would correspond to a fixed point for $p_{2}\left(\Lambda_{0}\right) \curvearrowright \overline{T_{2 k}}$, hence to a fixed point for $U\left(A_{2 k}\right) \curvearrowright \overline{T_{2 k}}$. We conclude that $\Lambda_{0} \curvearrowright T_{2 k} \times T_{2 k}$ is Roller minimal and the same argument also shows that it is Roller nonelementary.

One can easily check that $\Lambda_{0} \curvearrowright T_{2 k} \times T_{2 k}$ can be extended to an action of the whole $\Lambda$ by setting $\lambda g \cdot(x, y)=\left(p_{2}\left(\lambda g^{2}\right) \cdot y, p_{2}\left(g^{-1} \lambda g\right) \cdot x\right)$ for all $\lambda \in \Lambda_{0}$. This action also is Roller minimal and Roller nonelementary. We 
will show, however, that there exists no $\Lambda$-equivariant isometric embedding $j: Y \hookrightarrow \overline{T_{2 k}} \times \overline{T_{2 k}}$ of a median space $Y$ such that the action on $Y$ extends continuously to $U\left(A_{2 k}\right) \times U\left(A_{2 k}\right)$ by factoring through one of the factors.

Let $j: Y \hookrightarrow \overline{T_{2 k}} \times \overline{T_{2 k}}$ be a $\Lambda$-equivariant embedding; note that $j(Y)$ is entirely contained in a $\Lambda$-invariant component $Z \subseteq \overline{T_{2 k}} \times \overline{T_{2 k}}$. In particular, the previous discussion shows that $Z=T_{2 k} \times T_{2 k}$.

By Lemma 6.5 in [Bow13a, each wall of $j(Y)$ arises from a wall of $T_{2 k} \times T_{2 k}$, i.e. a wall of one of the two factors, see part 1 of Proposition 2.11. Since the two factors are exchanged by $g \in \Lambda$, we conclude that $Y$ splits as $Y_{1} \times Y_{2}$, with $\Lambda_{0} \curvearrowright Y$ preserving this decomposition and $g$ exchanging $Y_{1}$ and $Y_{2}$.

Suppose for the sake of contradiction that $\Lambda \curvearrowright Y$ extends to an action of $U\left(A_{2 k}\right) \times U\left(A_{2 k}\right)$ by factoring through one of the two factors. As in Example 4.7, we see that the extension cannot factor via $p_{1}$. However, since $p_{2}\left(\Lambda_{0}\right)$ is dense in $U\left(A_{2 k}\right)$ and $\Lambda_{0}$ preserves the splitting $Y=Y_{1} \times Y_{2}$, part 2 of Proposition 2.11 implies that an extension factoring through $p_{2}$ would also preserve the splitting $Y=Y_{1} \times Y_{2}$. This contradicts the fact that $g$ exchanges $Y_{1}$ and $Y_{2}$.

We conclude the section by proving Theorem $\mathrm{C}$.

Proof of Theorem $\mathbb{C}$. We begin by observing that part 2 follows from part 1 and Proposition 2.19. Now, suppose for the sake of contradiction that $\Gamma$ admits a Roller nonelementary action on $X$. As in the proof of Theorem $\mathrm{G}$, we can assume that $X$ is irreducible and that $\Gamma \curvearrowright X$ is Roller minimal. Theorem 4.4 then yields a factor $G_{i}$, a closed median subalgebra $Y \subseteq X$ and actions $G_{i} \curvearrowright Y$ and $\Gamma \curvearrowright Y$. Without loss of generality, we can assume that $Y$ is the closure of $Y_{0}$ inside $X$, as in Remark 4.5.

Stabilisers of points of $Y_{0}$ are open in $G_{i}$, thus the identity component $G_{i}^{0}$ must fix $Y_{0}$ pointwise. As $Y_{0}$ is dense in $Y$, the entire action $G_{i}^{0} \curvearrowright Y$ vanishes and $G_{i} \curvearrowright Y$ descends to an action of the group $G_{i} / G_{i}^{0}$. Since $G_{i}$ satisfies condition $(*)$, Theorem $\mathrm{G}$ above and Corollary 6.5 in Fio18 imply that the action $G_{i} / G_{i}^{0} \curvearrowright Y$ is Roller elementary. However, by Lemma 2.6, the actions $\Gamma \curvearrowright Y$ and $G_{i} \curvearrowright Y$ are Roller nonelementary, a contradiction.

4.2. Homomorphisms to coarse median groups. We defined equivariantly coarse median groups in the introduction. Here we simply prove Corollary $\mathrm{E}$.

Proof of Corollary $E$, Fix a non-principal ultrafilter $\omega$ on $\mathbb{N}$ and let $H_{\omega}$ be the corresponding ultrapower of $H$. We endow $H$ with a word metric $d_{S}$ arising from a finite generating set $S \subseteq H$. Given $\underline{\lambda}=\left(\lambda_{n}\right) \in \mathbb{R}_{+}^{\mathbb{N}}$, we denote by Cone $_{\omega}(H, \underline{\lambda})$ the asymptotic cone obtained by taking all basepoints at the identity and $\underline{\lambda}$ as sequence of scaling factors. Let $d$ denote the metric that $d_{S}$ induces on Cone $_{\omega}(H, \underline{\lambda})$; it is a geodesic metric and it is preserved by the natural action $H_{\omega} \curvearrowright \operatorname{Cone}_{\omega}(H, \underline{\lambda})$.

If $\lambda_{n} \rightarrow+\infty$, the coarse median on $H$ induces a structure of finite rank median algebra on $\operatorname{Cone}_{\omega}(H, \underline{\lambda})$, see Section 9 in [Bow13a]; we denote by 
$m$ the corresponding median map. The action $H_{\omega} \curvearrowright \operatorname{Cone}_{\omega}(H, \underline{\lambda})$ is by automorphisms of the median algebra structure. By Propositions 3.3 and 5.1 in [Zei16], we can endow $\operatorname{Cone}_{\omega}(H, \underline{\lambda})$ with a median metric $d_{m}$ that is biLipschitz equivalent to $d$ and preserved by the $H_{\omega}$-action; furthermore, the median algebra structure associated to $d_{m}$ is given by the map $m$.

Now suppose for the sake of contradiction that there exist pairwise nonconjugate homomorphisms $\phi_{n}: \Gamma \rightarrow H$, for $n \geq 0$; these correspond to a homomorphism $\Gamma \rightarrow H_{\omega}$, hence to an action on every asymptotic cone of $H$ that preserves the median metric $d_{m}$. The Bestvina-Paulin construction Bes88, Pau88, Pau91 provides us with a sequence $\mu_{n} \rightarrow+\infty$ such that, modifying each $\phi_{n}$ within its conjugacy class if necessary, the induced action $\Gamma \curvearrowright \operatorname{Cone}_{\omega}(H, \underline{\mu})$ has no global fixed point. This, however, contradicts Theorem C.

\section{Appendix A. Structure of UBS's.}

Let $X$ be a complete median space of finite rank $r$. We fix points $x \in X$ and $\xi \in \partial X$. Let $\Omega \subseteq \sigma_{\xi} \backslash \sigma_{x}$ be a minimal, reduced UBS (Definition 2.16).

Lemma A.1. Let $g \in I^{-s_{0} m_{\xi}} X$ be an isometry satisfying $g \Omega \sim \Omega$. Consider the UBS $\Omega_{1}:=\bigcap_{0 \leq i \leq r} g^{-i} \Omega \subseteq \Omega$.

(1) If $\chi_{\Omega}(g) \geq 0$, we have $g^{r !} \Omega_{1} \subseteq \Omega_{1}$ and $g^{r !} \mathfrak{h} \subseteq \mathfrak{h}$ for all $\mathfrak{h} \in \Omega_{1}$.

(2) If $\chi_{\Omega}(g)=0$, we have $g^{r !} \Omega_{1}=\Omega_{1}$ and $g^{r !} \mathfrak{h}=\mathfrak{h}$ for all $\mathfrak{h} \in \Omega_{1}$.

Proof. Observe that $\Omega_{1} \sim \Omega$; we fix a diverging chain $\left(\mathfrak{k}_{n}\right)_{n \geq 0}$ in $\Omega_{1}$. If $\mathfrak{h} \in \Omega_{1}$, the halfspaces $g^{i} \mathfrak{h}$ with $0 \leq i \leq r$ all lie in $\Omega \subseteq \sigma_{\xi} \backslash \sigma_{x}$ and cannot be pairwise transverse; thus, there exists $0 \leq i \leq r$ such that either $g^{i} \mathfrak{h} \subseteq \mathfrak{h}$, or $g^{i} \mathfrak{h} \supseteq \mathfrak{h}$. Hence, for every $\mathfrak{h} \in \Omega_{1}$ we either have $g^{r !} \mathfrak{h} \subseteq \mathfrak{h}$ or $g^{r !} \mathfrak{h} \supseteq \mathfrak{h}$.

Suppose that $g^{r !} \mathfrak{h}_{0} \subsetneq \mathfrak{h}_{0}$ for some $\mathfrak{h}_{0} \in \Omega_{1}$; set $\mathfrak{h}_{k}:=g^{k r !} \mathfrak{h}_{0}$. For each $k \geq 0$, a cofinite subchain of $\left\{g^{-k r !} \mathfrak{k}_{n}\right\}_{n \geq 0}$ is a diverging chain in $\Omega_{1}$, as $g \Omega_{1} \sim \Omega_{1}$. Hence, for each $k \geq 0$ we have $\mathfrak{h}_{0} \supseteq g^{-k r !} \mathfrak{k}_{n}$ if $n$ is sufficiently large, since $\Omega$ is reduced; in particular $\mathfrak{h}_{0} \supseteq \mathfrak{h}_{k} \supseteq \mathfrak{k}_{n}$. We conclude that each $\mathfrak{h}_{k}$ lies in $\Omega_{1}$; Proposition 2.1 guarantees that $\left(\mathfrak{h}_{k}\right)_{k \geq 0}$ is a diverging chain.

Let $\Xi \subseteq \Omega_{1}$ be the inseparable closure of $\left\{\mathfrak{h}_{k}\right\}_{k \geq 0}$; it is a UBS equivalent to $\Omega$ and it satisfies $g^{r !} \Xi \subseteq \Xi$. Observe that, for each $k \geq 0$,

$$
k r ! \cdot \chi_{\Omega}(g)=\chi_{\Omega}\left(g^{k r !}\right)=\chi_{\Xi}\left(g^{k r !}\right)=\widehat{\nu}\left(\Xi \backslash g^{k r !} \Xi\right) \geq d\left(\mathfrak{h}_{k}, \mathfrak{h}_{0}^{*}\right) ;
$$

since $d\left(\mathfrak{h}_{k}, \mathfrak{h}_{0}^{*}\right)>0$ for some $k \geq 0$, we conclude that $\chi_{\Omega}(g)>0$. The same argument applied to $g^{-1}$ shows that $\chi_{\Omega}(g)<0$ if there exists $\mathfrak{h}_{0} \in \Omega_{1}$ with $g^{r !} \mathfrak{h}_{0} \supsetneq \mathfrak{h}_{0}$.

This proves part 2 and shows that $g^{r !} \mathfrak{h} \subseteq \mathfrak{h}$ for all $\mathfrak{h} \in \Omega_{1}$ if $\chi_{\Omega}(g)>0$. In the latter case, for every $\mathfrak{h} \in \Omega_{1}$ we have $\mathfrak{h} \supseteq g^{r ! \mathfrak{h}} \supseteq \mathfrak{k}_{n}$ for sufficiently large $n$, since $\Omega$ is reduced; thus, $g^{r ! \mathfrak{h}} \in \Omega_{1}$ and $g^{r !} \Omega_{1} \subseteq \Omega_{1}$.

Corollary A.2. Let $g \in$ Isom $_{\xi} X$ be an isometry satisfying $g \Omega \sim \Omega$. Define $\Omega_{1}$ as in the previous lemma and consider the $U B S \Omega_{2}:=\bigcap_{1 \leq i \leq r !} g^{i-1} \Omega_{1}$.

(1) If $\chi_{\Omega}(g) \geq 0$, we have $g \Omega_{2} \subseteq \Omega_{2}$. 
(2) If $\chi_{\Omega}(g)=0$, we have $g \Omega_{2}=\Omega_{2}$.

In the rest of the appendix, we also consider a compact subset $K \subseteq \operatorname{Isom}_{\xi} X$ such that $g \Omega \sim \Omega$ for every $g \in K$.

Lemma A.3. (1) There exists a constant $C_{1}=C_{1}(\Omega, K)$ such that every $\mathfrak{h} \in \Omega$ with $d(x, \mathfrak{h})>C_{1}$ lies in $g \Omega$ for every $g \in K$.

(2) If $\Xi \subseteq \sigma_{\xi} \backslash \sigma_{x}$ is a minimal, reduced $U B S$ such that $\Xi \nsim \Omega$ and $g \Xi \sim \Xi$ for all $g \in K$, there exists a $U B S \bar{\Omega} \subseteq \Omega$ that is disjoint from $g \Xi$ for all $g \in K$.

Proof. Let $\left(\mathfrak{h}_{n}\right)_{n \geq 0}$ be a diverging chain in $\Omega$ with $\mathfrak{h}_{0}$ thick. For every $g \in K$, a cofinite subchain of $\left(g^{-1} \mathfrak{h}_{n}\right)_{n \geq 0}$ is contained in $\Omega$, as $g \Omega \sim \Omega$; since $\Omega$ is reduced, there exists $n(g) \geq 0$ so that $g^{-1} \mathfrak{h}_{n(g)} \subseteq \mathfrak{h}_{0}$. By Proposition 2.1 we can assume that $\widehat{\nu}\left(\mathscr{H}\left(g \mathfrak{h}_{0}^{*} \mid \mathfrak{h}_{n(g)}\right)\right)>0$ and Lemma 2.20 provides a neighbourhood $U(g)$ of $g$ in $K$ such that $\mathscr{H}\left(\gamma \mathfrak{h}_{0}^{*} \mid \xi\right) \cap \mathscr{H}\left(g \mathfrak{h}_{0}^{*} \mid \mathfrak{h}_{n(g)}\right) \neq \emptyset$ for all $\gamma \in U(g)$; in particular, $\mathfrak{h}_{n(g)} \subseteq \gamma \mathfrak{h}_{0}$ for all $\gamma \in U(g)$. There exist $g_{1}, \ldots, g_{k} \in K$ such that $K=U\left(g_{1}\right) \cup \ldots \cup U\left(g_{k}\right)$; if $N$ is the maximum of the $n\left(g_{i}\right)$, we have $\mathfrak{h}_{N} \subseteq g \mathfrak{h}_{0}$ for all $g \in K$. Let $\Omega_{N}$ be the inseparable closure of $\left\{\mathfrak{h}_{n}\right\}_{n \geq N}$. If $m \geq N$ and $g \in K$, we have $g \mathfrak{h}_{0} \supseteq \mathfrak{h}_{m} \supseteq g \mathfrak{h}_{n}$ for every sufficiently large $n$, since $\Omega$ is reduced. This shows that $\Omega_{N}$ is contained in $g \Omega$ for all $g \in K$. Since $\Omega_{N} \sim \Omega$, there exists a constant $C_{1}$ such that every $\mathfrak{h} \in \Omega$ with $d(x, \mathfrak{h})>C_{1}$ lies in $\Omega_{N}$.

To prove part 2 , let $C$ be the supremum of distances $d(x, \mathfrak{h})$ for $\mathfrak{h} \in \Omega \cap \Xi$; we have $C<+\infty$ since $\Omega \nsim \Xi$. Let $M$ be the maximum distance $d(x, g x)$ for $g \in K$ and consider $C^{\prime}:=\max \left\{C, C_{1}\left(\Xi, K^{-1}\right)+M\right\}$; we define $\bar{\Omega}$ to be the set of $\mathfrak{h} \in \Omega$ with $d(x, \mathfrak{h})>C^{\prime}$. If there existed $\mathfrak{h} \in \bar{\Omega} \cap g \Xi$ for some $g \in K$, we would have $g^{-1} \mathfrak{h} \in \Xi$ and $d\left(x, g^{-1} \mathfrak{h}\right)>C_{1}\left(\Xi, K^{-1}\right)$; thus, part 1 implies that $g^{-1} \mathfrak{h} \in g^{-1} \Xi$, i.e. $\mathfrak{h} \in \Xi$, and this contradicts the fact that $\mathfrak{h} \in \Omega$ and $d(x, \mathfrak{h})>C$.

Recall that we have introduced (in Section 3.1 after Proposition 3.4) the function $\alpha_{\Omega}: \mathscr{H} \rightarrow \mathbb{R}$, defined by the formula $\alpha_{\Omega}(\mathfrak{h}):=\widehat{\nu}(\mathscr{H}(x \mid \mathfrak{h}) \cap \Omega)$ and the sets $\Omega_{c}:=\left\{\mathfrak{h} \in \Omega \mid \alpha_{\Omega}(\mathfrak{h}) \leq c\right\}$. Observe that $\alpha_{\Omega}(\mathfrak{k}) \leq \alpha_{\Omega}(\mathfrak{h})$ whenever $\mathfrak{h} \subseteq \mathfrak{k}$; in particular $\alpha_{\Omega}$ is measurable. We have $\alpha_{\Omega}(\mathfrak{h}) \leq \widehat{\nu}(\mathscr{H}(x \mid \mathfrak{h}))=d(x, \mathfrak{h})$ for all $\mathfrak{h} \in \mathscr{H}$.

We say that $\Omega$ is small if $\widehat{\nu}(\Omega)<+\infty$; otherwise, $\Omega$ is large. If $X$ is a CAT $(0)$ cube complex, every UBS is large; an example of a small UBS in a rank two median space appears in Figure 3 of [Fio17]. If $\Omega$ is small, we have $\chi_{\Omega}(h)=0$ for every isometry $h$ fixing $[\Omega]$. Note that the supremum of $\alpha_{\Omega}$ is precisely $\widehat{\nu}(\Omega)$.

Lemma A.4. Let $\mathfrak{h}_{n} \in \Omega$ be halfspaces with $\mathfrak{h}_{n+1} \subseteq \mathfrak{h}_{n}$ for all $n \geq 0$. Then, $\alpha_{\Omega}\left(\mathfrak{h}_{n}\right) \rightarrow \widehat{\nu}(\Omega)$ if and only if $\left(\mathfrak{h}_{n}\right)_{n \geq 0}$ is a diverging chain of halfspaces.

Proof. The fact that $\alpha_{\Omega}\left(\mathfrak{h}_{n}\right) \rightarrow \widehat{\nu}(\Omega)$ if $\left(\mathfrak{h}_{n}\right)_{n \geq 0}$ is a diverging chain follows from the fact that $\Omega$ is reduced. For the other implication, let $\left(\mathfrak{k}_{m}\right)_{m \geq 0}$ be a diverging chain in $\Omega$. Since $\alpha_{\Omega}(\mathfrak{h}) \leq d(x, \mathfrak{h})$, it suffices to consider the case 
when $\Omega$ is small. For every $m \geq 0$, the set $\left\{\mathfrak{j} \in \Omega \mid \mathfrak{j} \subseteq \mathfrak{k}_{m}\right\}$ has measure $a_{m}>0$ by Proposition 2.1. For large $n$ we have $\alpha_{\Omega}\left(\mathfrak{h}_{n}\right)>\widehat{\nu}(\Omega)-a_{m}$, hence there exists $\mathfrak{j} \subseteq \mathfrak{k}_{m}$ such that $j \in \mathscr{H}\left(x \mid \mathfrak{h}_{n}\right)$; in particular, $\mathfrak{h}_{n} \subseteq \mathfrak{k}_{m}$. Since $m$ is arbitrary, this shows that $\left(\mathfrak{h}_{n}\right)_{n \geq 0}$ is a diverging chain.

Lemma A.5. (1) For every $0 \leq c<\widehat{\nu}(\Omega)$, the set $\Omega \backslash \Omega_{c}$ is a UBS.

(2) For all $c \geq 0$, we have $\widehat{\nu}\left(\Omega_{c}\right) \leq r c$.

Proof. Since $\Omega$ is reduced, any $\mathfrak{h} \in \Omega \backslash \Omega_{c}$ contains almost every halfspace in any diverging chain in $\Omega$; this provides a diverging chain in $\Omega \backslash \Omega_{c}$. Inseparability follows from the monotonicity of $\alpha_{\Omega}$.

To prove part 2, we decompose $\Omega_{c}=\mathcal{C}_{1} \sqcup \ldots \sqcup \mathcal{C}_{k}$ as in Lemma 2.2. If $\mathfrak{h}, \mathfrak{k} \in \mathcal{C}_{i}$ and $\mathfrak{h} \subseteq \mathfrak{k}$, we have $c \geq \alpha_{\Omega}(\mathfrak{h}) \geq \widehat{\nu}\left(\mathscr{H}\left(\mathfrak{k}^{*} \mid \mathfrak{h}\right)\right)$. Hence, Lemma 2.27 in [Fio17] implies that the inseparable closure of $\mathcal{C}_{i}$ has measure at most $c$. We conclude that $\widehat{\nu}\left(\Omega_{c}\right) \leq k c \leq r c$.

Lemma A.6. Assume that $g \Omega \subseteq \Omega$ for all $g \in K$.

(1) For all $\mathfrak{h} \in \Omega$ and $g \in K$, we have

$$
-d(x, g x)-\chi_{\Omega}(g) \leq \alpha_{\Omega}\left(g^{-1} \mathfrak{h}\right)-\alpha_{\Omega}(\mathfrak{h}) \leq d(x, g x)
$$

in particular $\left\|g \alpha_{\Omega}-\alpha_{\Omega}\right\|_{\infty} \leq C_{2}$ for some constant $C_{2}=C_{2}(\Omega, K)$.

(2) For every $c>0$, we have $g \Omega_{c} \subseteq \Omega_{c+C_{2}}$.

Proof. Indeed,

$$
\begin{aligned}
\alpha_{\Omega}\left(g^{-1} \mathfrak{h}\right) & \leq \widehat{\nu}\left(\mathscr{H}\left(x \mid g^{-1} x\right)\right)+\widehat{\nu}\left(\mathscr{H}\left(g^{-1} x \mid g^{-1} \mathfrak{h}\right) \cap \Omega\right) \\
& =d\left(x, g^{-1} x\right)+\widehat{\nu}(\mathscr{H}(x \mid \mathfrak{h}) \cap g \Omega) \leq d(x, g x)+\alpha_{\Omega}(\mathfrak{h}),
\end{aligned}
$$

and

$$
\begin{aligned}
\alpha_{\Omega}(\mathfrak{h}) & \leq \widehat{\nu}(\mathscr{H}(x \mid g x))+\widehat{\nu}(\mathscr{H}(g x \mid \mathfrak{h}) \cap \Omega) \\
& =d(x, g x)+\widehat{\nu}\left(\mathscr{H}\left(x \mid g^{-1} \mathfrak{h}\right) \cap g^{-1} \Omega\right) \\
& \leq d(x, g x)+\widehat{\nu}\left(\mathscr{H}\left(x \mid g^{-1} \mathfrak{h}\right) \cap \Omega\right)+\widehat{\nu}\left(g^{-1} \Omega \backslash \Omega\right) \\
& =d(x, g x)+\alpha_{\Omega}\left(g^{-1} \mathfrak{h}\right)+\chi_{\Omega}(g) .
\end{aligned}
$$

We then take $C_{2}$ to be the maximum of $d(x, g x)+\chi_{\Omega}(g)$ for $g \in K$; this exists due to Proposition 2.19. Regarding part 2, observe that, if $\mathfrak{h} \in \Omega_{c}$,

$$
\alpha_{\Omega}(g \mathfrak{h})=\alpha_{\Omega}(\mathfrak{h})+\left(\alpha_{\Omega}(g \mathfrak{h})-g \alpha_{\Omega}(g \mathfrak{h})\right) \leq c+C_{2} .
$$

Lemma A.7. Assume that $\Omega$ is strongly reduced.

(1) For every $d \geq 0$, there exists a constant $C_{3}=C_{3}(\Omega, d)$ such that $\mathfrak{h} \subseteq \mathfrak{k}$ for all $\mathfrak{h}, \mathfrak{k} \in \Omega$ with $d(x, \mathfrak{h})>C_{3}$ and $d(x, \mathfrak{k}) \leq d$.

(2) If $\Xi \subseteq \Omega$ is a UBS and $d(x, \mathfrak{k}) \leq d$ for all $\mathfrak{k} \in \Omega \backslash \Xi$, we have $\alpha_{\Omega}(\mathfrak{h})-\alpha_{\Xi}(\mathfrak{h})=\widehat{\nu}(\Omega \backslash \Xi)$ for all $\mathfrak{h} \in \Omega$ with $d(x, \mathfrak{h})>C_{3}(\Omega, d)$. 
Proof. Decompose $\Omega=\mathcal{C}_{1} \sqcup \ldots \sqcup \mathcal{C}_{k}$, where each $\mathcal{C}_{i}$ is totally ordered by inclusion and contains a diverging chain. Pick halfspaces $\mathfrak{k}_{i} \in \mathcal{C}_{i}$ with $d\left(x, \mathfrak{k}_{i}\right)>d$. By part 3 of Lemma 2.18, the halfspace $\mathfrak{k}_{i}$ cannot be transverse to a diverging chain in $\Omega$; thus, part 2 of Lemma 2.2 guarantees that the halfspaces of $\Omega$ that are transverse to $\mathfrak{k}_{i}$ lie at uniformly bounded distance from $x$. We conclude that there exists $C_{3}$ such that every $\mathfrak{h} \in \Omega$ with $d(x, \mathfrak{h})>C_{3}$ is contained in each $\mathfrak{k}_{i}$, hence also in each $\mathfrak{k} \in \Omega$ with $d(x, \mathfrak{k}) \leq d$. This proves part 1; part 2 is an immediate consequence.

In the rest of the section, we assume that $\Omega$ is minimal and strongly reduced.

Lemma A.8. Suppose that $\chi_{\Omega}(g) \geq 0$ for all $g \in K$.

(1) There exists a constant $C_{4}=C_{4}(\Omega, K)$ such that, for every halfspace $\mathfrak{h} \in \Omega$ with $d(x, \mathfrak{h})>C_{4}$ and every $g \in K$, we have $\alpha_{\Omega}(g \mathfrak{h}) \geq \alpha_{\Omega}(\mathfrak{h})$.

(2) There exists a constant $C_{5}=C_{5}(\Omega, K)<\widehat{\nu}(\Omega)$ such that we have $g\left(\Omega \backslash \Omega_{c}\right) \subseteq \Omega \backslash \Omega_{c}$ and $\Omega_{c} \backslash g \Omega_{c}=\Omega \backslash g \Omega$ whenever $c>C_{5}$ and $g \in K$.

Proof. We first observe that, given a minimal UBS $\Xi \subseteq \sigma_{\xi} \backslash \sigma_{x}$ and an isometry $g \in \operatorname{Isom}_{\xi} X$ with $g \Xi \subseteq \Xi$, we have:

$$
\begin{aligned}
\alpha_{\Xi} & (g \mathfrak{h})-\alpha_{\Xi}(\mathfrak{h})=\widehat{\nu}\left(\mathscr{H}\left(g^{-1} x \mid \mathfrak{h}\right) \cap g^{-1} \Xi\right)-\widehat{\nu}(\mathscr{H}(x \mid \mathfrak{h}) \cap \Xi) \\
& =\widehat{\nu}\left(\mathscr{H}\left(g^{-1} x \mid \mathfrak{h}\right) \cap \Xi\right)-\widehat{\nu}(\mathscr{H}(x \mid \mathfrak{h}) \cap \Xi)+\widehat{\nu}\left(\mathscr{H}\left(g^{-1} x \mid \mathfrak{h}\right) \cap\left(g^{-1} \Xi \backslash \Xi\right)\right) \\
& =-\widehat{\nu}\left(\mathscr{H}\left(x \mid g^{-1} x, \mathfrak{h}\right) \cap \Xi\right)+\widehat{\nu}\left(\mathscr{H}\left(g^{-1} x \mid \mathfrak{h}\right) \cap\left(g^{-1} \Xi \backslash \Xi\right)\right),
\end{aligned}
$$

since $\mathscr{H}\left(g^{-1} x \mid x\right) \cap \Xi=\emptyset$, as $\Xi \subseteq \sigma_{\xi} \backslash \sigma_{x}$. Note that:

$$
\mathscr{H}\left(x \mid g^{-1} x\right) \cap g \Xi=g^{-1}\left(\mathscr{H}(g x \mid x) \cap g^{2} \Xi\right) \subseteq g^{-1}(\mathscr{H}(g x \mid x) \cap \Xi)=\emptyset .
$$

Thus $\alpha_{\Xi}(g \mathfrak{h})-\alpha_{\Xi}(\mathfrak{h})$ equals:

$$
\begin{aligned}
& -\widehat{\nu}\left(\mathscr{H}\left(x \mid g^{-1} x, \mathfrak{h}\right) \cap(\Xi \backslash g \Xi)\right)+\widehat{\nu}\left(\mathscr{H}\left(g^{-1} x \mid \mathfrak{h}\right) \cap\left(g^{-1} \Xi \backslash \Xi\right)\right) \\
& \geq-\widehat{\nu}\left(\sigma_{x}^{*} \cap(\Xi \backslash g \Xi)\right)+\widehat{\nu}(\mathscr{H}(x \mid g \mathfrak{h}) \cap(\Xi \backslash g \Xi)) \\
& \geq-\widehat{\nu}\left(\left(\sigma_{x}^{*} \backslash \mathscr{H}(x \mid g \mathfrak{h})\right) \cap(\Xi \backslash g \Xi)\right) \geq-\widehat{\nu}(\{\mathfrak{k} \in \Xi \backslash g \Xi \mid g \mathfrak{h} \nsubseteq \mathbb{k}\}) .
\end{aligned}
$$

Now, consider for each $g \in K$ the set $\Omega_{2}(g):=\bigcap_{-r+1 \leq i \leq r !} g^{i-1} \Omega$ as in Corollary A.2. we have $g \Omega_{2}(g) \subseteq \Omega_{2}(g)$ and, by part 1 of Lemma A.3, there exists a constant $C_{1}$ such that each $\mathfrak{h} \in \Omega$ with $d(x, \mathfrak{h})>C_{1}$ lies in $g \Omega_{2}(g)$ for every $g \in K$. By part 1 of Lemma A.7, if $d(x, g \mathfrak{h})>C_{3}\left(\Omega, C_{1}\right)$ we have

$$
\alpha_{\Omega_{2}(g)}(g \mathfrak{h})-\alpha_{\Omega_{2}(g)}(\mathfrak{h}) \geq-\widehat{\nu}\left(\left\{\mathfrak{k} \in \Omega_{2}(g) \backslash g \Omega_{2}(g) \mid g \mathfrak{h} \not \subset \mathfrak{k}\right\}\right)=0 .
$$

By part 2 of Lemma A.7, if $d(x, \mathfrak{h})>C_{3}\left(\Omega, C_{1}\right)$ and $d(x, g \mathfrak{h})>C_{3}\left(\Omega, C_{1}\right)$, we have

$$
\alpha_{\Omega}(g \mathfrak{h})-\alpha_{\Omega}(\mathfrak{h})=\alpha_{\Omega_{2}(g)}(g \mathfrak{h})-\alpha_{\Omega_{2}(g)}(\mathfrak{h}) \geq 0 .
$$

We conclude that $\alpha_{\Omega}(g \mathfrak{h}) \geq \alpha_{\Omega}(\mathfrak{h})$ whenever $d(x, \mathfrak{h})>C_{4}:=C_{3}\left(\Omega, C_{1}\right)+M$, where $M$ is the maximum of the distances $d(x, g x)$ for $g \in K$. 
We now prove part 2. Let $C_{4}$ be as in part 1. Part 1 of Lemma A.3 provides a constant $C_{1}^{\prime}$ such that each $\mathfrak{h} \in \Omega$ with $d(x, \mathfrak{h})>C_{1}^{\prime}$ lies in $g \Omega \cap g^{-1} \Omega$ for every $g \in K$. Let $C_{5}$ be the supremum of the values $\alpha_{\Omega}(\mathfrak{h})$ for $\mathfrak{h} \in \Omega$ with $d(x, \mathfrak{h}) \leq \max \left\{C_{4}, C_{1}^{\prime}\right\}$; by Lemma A.4 we have $C_{5}<\widehat{\nu}(\Omega)$. If $c>C_{5}$, any $\mathfrak{h} \in \Omega \backslash \Omega_{c}$ satisfies $d(x, \mathfrak{h})>\max \left\{C_{4}, C_{1}^{\prime}\right\}$, hence $\alpha_{\Omega}(g \mathfrak{h}) \geq \alpha_{\Omega}(\mathfrak{h})>c$ and $g \mathfrak{h} \in \Omega$; in particular $g\left(\Omega \backslash \Omega_{c}\right) \subseteq \Omega \backslash \Omega_{c}$.

Observe that $(\Omega \backslash g \Omega) \cap g \Omega_{c} \subseteq g\left(\Omega_{c} \backslash \Omega\right)=\emptyset$ and $\Omega \backslash g \Omega \subseteq \Omega_{c}$, by our choice of the constant $C_{1}^{\prime}$; thus, $\Omega \backslash g \Omega \subseteq \Omega_{c} \backslash g \Omega_{c}$. Conversely, it is clear that $\Omega_{c} \backslash g \Omega_{c} \subseteq \Omega$ and

$$
\left(\Omega_{c} \backslash g \Omega_{c}\right) \cap g \Omega=\Omega_{c} \cap g\left(\Omega \backslash \Omega_{c}\right) \subseteq \Omega_{c} \cap\left(\Omega \backslash \Omega_{c}\right)=\emptyset .
$$

Consider now, for $c>0$, the functions $F_{c, \Omega}:=-\left(1-\frac{\alpha_{\Omega}}{c}\right) \mathbb{1}_{\Omega_{c}}$ in $L^{2}(\mathscr{H}, \widehat{\nu})$.

Lemma A.9. Assume that $g \Omega \subseteq \Omega$ for all $g \in K$. For every $\epsilon$, there exists a constant $C_{\epsilon}=C_{\epsilon}(\Omega, K)<+\infty$ such that $\left\|(g-i d) F_{c, \Omega}-\mathbb{1}_{\Omega \backslash g \Omega}\right\|_{2}<\epsilon$ for all $g \in K$ and all $c \geq C_{\epsilon}$. If instead $g \Omega \supseteq \Omega$ for all $g \in K$, we have $\left\|(g-i d) F_{c, \Omega}+\mathbb{1}_{g \Omega \backslash \Omega}\right\|_{2}<\epsilon$ for $c \geq C_{\epsilon}$.

Proof. Observe that:

$$
\begin{aligned}
&(g-\mathrm{id}) F_{c, \Omega}=-\left(1-\frac{g \alpha_{\Omega}}{c}\right) \mathbb{1}_{g \Omega_{c}}+\left(1-\frac{\alpha_{\Omega}}{c}\right) \mathbb{1}_{\Omega_{c}} \\
&=-\left(1-\frac{g \alpha_{\Omega}}{c}\right) \mathbb{1}_{g \Omega_{c} \backslash \Omega_{c}}+\left(1-\frac{\alpha_{\Omega}}{c}\right) \mathbb{1}_{\Omega_{c} \backslash g \Omega_{c}}+\frac{g \alpha_{\Omega}-\alpha_{\Omega}}{c} \mathbb{1}_{g \Omega_{c} \cap \Omega_{c} .}
\end{aligned}
$$

We will analyse the three summands separately. By Lemma A.6, we have

$$
-\frac{2 C_{2}}{c} \leq\left(1-\frac{\alpha_{\Omega}(\mathfrak{h})+C_{2}}{c}\right) \leq\left(1-\frac{g \alpha_{\Omega}(\mathfrak{h})}{c}\right) \leq\left(1-\frac{\alpha_{\Omega}(\mathfrak{h})-C_{2}}{c}\right)<\frac{C_{2}}{c},
$$

for each $\mathfrak{h} \in g \Omega_{c} \backslash \Omega_{c} \subseteq \Omega_{c+C_{2}} \backslash \Omega_{c}$. By part 2 of Lemma A.5.

$$
\left\|\left(1-\frac{g \alpha_{\Omega}}{c}\right) \mathbb{1}_{g \Omega_{c} \backslash \Omega_{c}}\right\|_{2} \leq \frac{2 C_{2}}{c} \cdot \widehat{\nu}\left(g \Omega_{c} \backslash \Omega_{c}\right)^{1 / 2} \leq \frac{2 C_{2}\left(r\left(c+C_{2}\right)\right)^{1 / 2}}{c} \underset{c \rightarrow+\infty}{\longrightarrow} 0 .
$$

By part 1 of Lemma A.3 there exists a constant $C_{1}$ such that each $\mathfrak{h} \in \Omega$ with $d(x, \mathfrak{h})>C_{1}$ lies in $g \Omega$ for every $g \in K$; in particular, if $\mathfrak{k} \in \Omega \backslash g \Omega$ for some $g \in K$, then $\alpha_{\Omega}(\mathfrak{k}) \leq d(x, \mathfrak{k}) \leq C_{1}$. By part 2 of Lemma A.8, if $c \geq C_{5}$ we have $\Omega_{c} \backslash g \Omega_{c}=\Omega \backslash g \Omega$ and

$$
\begin{aligned}
& \left\|\left(1-\frac{\alpha_{\Omega}}{c}\right) \mathbb{1}_{\Omega_{c} \backslash g \Omega_{c}}-\mathbb{1}_{\Omega \backslash g \Omega}\right\|_{2}=\left\|\left(\mathbb{1}_{\Omega_{c} \backslash g \Omega_{c}}-\mathbb{1}_{\Omega \backslash g \Omega}\right)-\frac{\alpha_{\Omega}}{c} \mathbb{1}_{\Omega_{c} \backslash g \Omega_{c}}\right\|_{2} \\
& =\left\|\frac{\alpha_{\Omega}}{c} \mathbb{1}_{\Omega \backslash g \Omega}\right\|_{2} \leq \frac{C_{1}}{c} \cdot \widehat{\nu}(\Omega \backslash g \Omega)^{1 / 2} \leq \frac{C_{1} M^{1 / 2}}{c} \underset{c \rightarrow+\infty}{\longrightarrow} 0,
\end{aligned}
$$

where $M$ is the maximum of $\chi_{\Omega}(g)$ for $g \in K$, which exists by Proposition 2.19. Finally, by part 2 of Lemma A.5 and part 1 of Lemma A.6.

$$
\left\|\frac{g \alpha_{\Omega}-\alpha_{\Omega}}{c} \mathbb{1}_{g \Omega_{c} \cap \Omega_{c}}\right\|_{2} \leq \frac{C_{2}}{c} \cdot \widehat{\nu}\left(\Omega_{c}\right)^{1 / 2} \leq \frac{C_{2}(c r)^{1 / 2}}{c} \underset{c \rightarrow+\infty}{\longrightarrow} 0 .
$$


If instead $g \Omega \supseteq \Omega$ for all $g \in K$, the previous discussion shows that, for large $c$, we have $\left\|\left(g^{-1}-\mathrm{id}\right) F_{c, \Omega}-\mathbb{1}_{\Omega \backslash g^{-1} \Omega}\right\|_{2}<\epsilon$; the conclusion follows by applying $g$.

Lemma A.10. Let $\Xi \subseteq \sigma_{\xi} \backslash \sigma_{x}$ be a $U B S$ and let $\Xi^{1}, \ldots, \Xi^{k} \subseteq \Xi$ be pairwise inequivalent, reduced $U B S^{\prime}$ 's representing all minimal equivalence classes of $U B S$ 's almost contained in $\Xi$; for every $i$, set $\sigma_{i}:=\widehat{\nu}\left(\Xi^{i}\right)$. There exist increasing sequences $\left(c_{n}^{(i)}\right)_{n \geq 0}$ such that

- $c_{n}^{(i)} \rightarrow \sigma_{i}$ for all $i=1, \ldots, k$;

- $\left(\Xi^{1} \backslash \Xi_{c_{n}^{(1)}}^{1}\right) \cup \ldots \cup\left(\Xi^{k} \backslash \Xi_{c_{n}^{(k)}}^{k}\right)$ is inseparable for all $n \geq 0$.

Proof. We proceed by induction on $k$. If $k=1$, the lemma is immediate. Suppose that $k \geq 2$; without loss of generality, we can assume that $\Xi^{k}$ corresponds to a vertex with no incoming edges in the full subgraph of $\mathcal{G}(\xi)$ with vertices $\left[\Xi^{1}\right], \ldots,\left[\Xi^{k}\right]$. Fix $\epsilon>0$; we will construct $c^{(i)}>\sigma_{i}-\epsilon$ satisfying the inseparability condition.

Pick a diverging chain $\left(\mathfrak{h}_{n}^{(i)}\right)_{n \geq 0}$ in each $\Xi^{i}$; up to replacing $\left(\mathfrak{h}_{n}^{(k)}\right)_{n \geq 0}$ with a cofinite subchain, we can assume that, for each $i \leq k-1$ and each $m \geq 0$, the halfspace $\mathfrak{h}_{m}^{(k)}$ is transverse to $\mathfrak{h}_{n}^{(i)}$ for almost every $n$. By Lemma A.4. there exists $c^{(k)}>\sigma_{k}-\epsilon$ such that $\Xi^{k} \backslash \Xi_{c^{(k)}}^{k}$ is contained in the inseparable closure of $\left\{\mathfrak{h}_{n}^{(k)}\right\}_{n \geq 0}$. As a consequence, for every $\mathfrak{h} \in \Xi^{k} \backslash \Xi_{c^{(k)}}^{k}$ and every $i \leq k-1$, the halfspaces $\mathfrak{h}$ and $\mathfrak{h}_{n}^{(i)}$ are transverse for almost every $n$.

Halfspaces lying in the inseparable closure of $\Xi^{1} \cup \ldots \cup \Xi^{k}$, but in neither of the $\Xi^{i}$, are at uniformly bounded distance from $x$, by part 3 of Proposition 2.17, say that these distances are bounded above by $M<+\infty$. Enlarge $M$ so that all $\mathfrak{j} \in \Xi^{k}$ with $d(x, \mathfrak{j})>M$ lie in $\Xi^{k} \backslash \Xi_{c^{(k)}}^{k}$. By part 4 of Proposition 2.17 there exists a UBS contained in $\Xi$ such that $\left[\Xi^{1}\right], \ldots,\left[\Xi^{k-1}\right]$ are all the equivalence classes of minimal UBS's almost contained in $\Xi$. The inductive hypothesis and Lemma A.4 imply that we can find $c^{(i)}>\sigma_{i}-\epsilon$ so that $\left(\Xi^{1} \backslash \Xi_{c^{(1)}}^{1}\right) \cup \ldots \cup\left(\Xi^{k-1} \backslash \Xi_{c^{(k-1)}}^{k-1}\right)$ is inseparable and $d(x, \mathfrak{h})>M$ for all $\mathfrak{h} \in \Xi^{i} \backslash \Xi_{c^{(i)}}^{i}$ with $i \leq k-1$.

Now, if $\left(\Xi^{1} \backslash \Xi_{c^{(1)}}^{1}\right) \cup \ldots \cup\left(\Xi^{k} \backslash \Xi_{c^{(k)}}^{k}\right)$ were not inseparable, there would exist $\mathfrak{j} \in \mathscr{H}$ such that $\mathfrak{k}_{u} \subseteq \mathfrak{j} \subseteq \mathfrak{k}_{v}$, for halfspaces $\mathfrak{k}_{u} \in\left(\Xi^{u} \backslash \Xi_{c^{(u)}}^{u}\right)$ and $\mathfrak{k}_{v} \in\left(\Xi^{v} \backslash \Xi_{c^{(v)}}^{v}\right)$, but $\mathfrak{j}$ would not lie in any of the $\Xi^{i} \backslash \Xi_{c^{(i)}}^{i}$. In particular, $u \neq v$ and $k \in\{u, v\}$; observe that $v \neq k$, otherwise $\mathfrak{k}_{v} \in \Xi^{k} \backslash \Xi_{c^{(k)}}^{k}$ would not be transverse to diverging chains in $\Xi^{u}$. Thus, $u=k$; moreover, for all $i \leq k-1$, the halfspace $\mathfrak{j}$ must be transverse to $\mathfrak{h}_{n}^{(i)}$ for almost every $n$, since $v \leq k-1$ and $\mathfrak{j}$ does not lie in $\left(\Xi^{1} \backslash \Xi_{c^{(1)}}^{1}\right) \cup \ldots \cup\left(\Xi^{k-1} \backslash \Xi_{c^{(k-1)}}^{k-1}\right)$, which is inseparable. Since $d(x, \mathfrak{j}) \geq d\left(x, \mathfrak{k}_{v}\right)>M$, we have $\mathfrak{j} \in \Xi^{1} \cup \ldots \cup \Xi^{k}$; the fact 
that each $\Xi_{i}$ is reduced implies that $j \in \Xi^{k}$. By our choice of $M$, we have $\mathfrak{j} \in \Xi^{k} \backslash \Xi_{c^{(k)}}^{k}$, a contradiction.

Lemma A.11. There exists a $U B S \Xi_{\xi} \subseteq \sigma_{\xi} \backslash \sigma_{x}$ such that every $U B S \Xi \subseteq \Xi_{\xi}$ with $\Xi \sim \Xi_{\xi}$ is of the form $\sigma_{\xi} \backslash \sigma_{y}$ for some $y \in X$, up to a null set.

Proof. Let $\Xi_{1}, \ldots, \Xi_{k} \subseteq \sigma_{\xi} \backslash \sigma_{x}$ be pairwise inequivalent minimal UBS's representing all minimal elements of $\overline{\mathcal{U}}(\xi)$; we can assume that they are all reduced by Lemma 2.18. Halfspaces in $\sigma_{\xi} \backslash \sigma_{x}$ that are transverse to a diverging chain in each $\Xi_{i}$ lie at uniformly bounded distance from $x$, by part 3 of Proposition 2.17, say that these distances are bounded above by $M<+\infty$. Let $\Xi_{\xi}$ consist of all $\mathfrak{h} \in \sigma_{\xi} \backslash \sigma_{x}$ with $d(x, \mathfrak{h})>M$; it is a UBS equivalent to $\sigma_{\xi} \backslash \sigma_{x}$.

Observe that, if $\Xi \subseteq \Xi_{\xi}$ is a UBS equivalent to $\Xi_{\xi}$ and $\xi \in \widetilde{\mathfrak{h}}$ for halfspaces $\mathfrak{h} \subseteq \mathfrak{k} \in \Xi$, then $\mathfrak{h} \in \Xi$. Indeed, otherwise $\mathfrak{h}$ would not contain any halfspace of $\Xi$, by inseparability, and it would therefore be transverse to a diverging chain in each of the $\Xi_{i}$; hence $d(x, \mathfrak{k}) \leq d(x, \mathfrak{h}) \leq M$, a contradiction.

Now, given $\Xi \subseteq \Xi_{\xi}$, consider the set $\sigma:=\left(\sigma_{\xi} \backslash \Xi\right) \sqcup \Xi^{*} \subseteq \mathscr{H}$. Observe that $\sigma$ is an ultrafilter. Indeed, since $\sigma_{\xi} \backslash \Xi \subseteq \sigma_{\xi}$ and $\Xi^{*} \subseteq \sigma_{x}$, it suffices to check that $\mathfrak{h} \cap \mathfrak{k} \neq \emptyset$ whenever $\mathfrak{h} \in \sigma_{\xi} \backslash \Xi$ and $\mathfrak{k} \in \Xi^{*}$. If such halfspaces were disjoint, we would have $\xi \in \widetilde{\mathfrak{h}}$ and $\mathfrak{h} \subseteq \mathfrak{k}^{*} \in \Xi$, contradicting the observation we made above since $\mathfrak{h} \notin \Xi$.

Finally, by Lemma 4.3 in [Fio18, we can decompose $\sigma_{\xi} \backslash \sigma_{x}=\Xi \sqcup \Sigma$ for some set $\Sigma$ with $\widehat{\nu}(\Sigma)<+\infty$; in particular, $\sigma_{x} \backslash \sigma_{\xi}=\Xi^{*} \sqcup \Sigma^{*}$. Since $\Xi$ and $\sigma_{x}$ are disjoint, we have $\sigma_{x} \backslash \sigma=\sigma_{x} \backslash\left(\sigma_{\xi} \cup \Xi^{*}\right)=\Sigma^{*}$, hence $\widehat{\nu}\left(\sigma_{x} \triangle \sigma\right)<+\infty$. Proposition 2.4 implies that there exists $y \in X$ such that $\sigma \triangle \sigma_{y}$ is null; thus, up to measure zero, $\sigma_{\xi} \backslash \sigma_{y}=\sigma_{\xi} \backslash \sigma=\Xi$.

We are now ready to prove Proposition 3.4

Proof of Proposition 3.4. Let $\Xi^{1}, \ldots, \Xi^{k} \subseteq \sigma_{\xi} \backslash \sigma_{x}$ be pairwise inequivalent UBS's representing all minimal elements of the poset $(\overline{\mathcal{U}}(\xi), \preceq)$; by Lemma 2.18, we can assume that each $\Xi^{i}$ is strongly reduced. Up to replacing each $\Xi^{i}$ with a smaller UBS, part 2 of Lemma A.3 guarantees that we can assume that $\Xi^{i} \cap \Xi^{j}=\emptyset$ and $\Xi^{i} \cap g \Xi^{j}$ for all $i \neq j$ and $g \in K$. By part 2 of Lemma A.8, we have $g\left(\Xi^{i} \backslash \Xi_{c}^{i}\right) \subseteq \Xi^{i} \backslash \Xi_{c}^{i}$ for all $C_{5}\left(\Xi^{i}, K\right) \leq c<\widehat{\nu}\left(\Xi^{i}\right)$ and all $g \in K$ with $\chi_{\Xi^{i}}(g) \geq 0$, while we have $g\left(\Xi^{i} \backslash \Xi_{c}^{i}\right) \supseteq \Xi^{i} \backslash \Xi_{c}^{i}$ if $\chi_{\Xi^{i}}(g) \leq 0$. Lemma A.10 provides constants $C_{5}\left(\Xi^{i}, K\right) \leq c_{i}<\widehat{\nu}\left(\Xi^{i}\right)$ such that $\Omega_{K}:=\left(\Xi^{1} \backslash \Xi_{c_{1}}^{1}\right) \cup \ldots \cup\left(\Xi^{k} \backslash \Xi_{c_{k}}^{k}\right)$ is inseparable. Thus $\Omega_{K}$ is a UBS equivalent to $\sigma_{\xi} \backslash \sigma_{x}$ by part 3 of Proposition 2.17. We conclude by Lemma A.11, enlarging the constants $c_{i}$ if necessary, so that $\Omega_{K} \subseteq \Xi_{\xi}$; this is possible by Lemma A.4.

\section{REFERENCES}

[ANWZ18] Goulnara Arzhantseva, Graham A. Niblo, Nick Wright, and Jiawen Zhang. A characterization for asymptotic dimension growth. Algebr. Geom. Topol., 18(1):493-524, 2018. 
$\left[\mathrm{BCG}^{+}\right.$09] Jacek Brodzki, Sarah J. Campbell, Erik Guentner, Graham A. Niblo, and Nick J. Wright. Property A and CAT(0) cube complexes. J. Funct. Anal., 256(5):1408-1431, 2009.

[BdlHV08] Bachir Bekka, Pierre de la Harpe, and Alain Valette. Kazhdan's property (T), volume 11 of New Mathematical Monographs. Cambridge University Press, Cambridge, 2008.

[BDS11a] Jason Behrstock, Cornelia Druţu, and Mark Sapir. Addendum: Median structures on asymptotic cones and homomorphisms into mapping class groups [mr2783135]. Proc. Lond. Math. Soc. (3), 102(3):555-562, 2011.

[BDS11b] Jason Behrstock, Cornelia Druţu, and Mark Sapir. Median structures on asymptotic cones and homomorphisms into mapping class groups. Proc. Lond. Math. Soc. (3), 102(3):503-554, 2011.

[Bes88] Mladen Bestvina. Degenerations of the hyperbolic space. Duke Math. J., 56(1):143-161, 1988.

[BF95] Mladen Bestvina and Mark Feighn. Stable actions of groups on real trees. Invent. Math., 121(2):287-321, 1995.

[BF14] Uri Bader and Alex Furman. Boundaries, rigidity of representations, and Lyapunov exponents. In Proceedings of the International Congress of Mathematicians-Seoul 2014. Vol. III, pages 71-96. Kyung Moon Sa, Seoul, 2014.

[BGM12] Uri Bader, Tsachik Gelander, and Nicolas Monod. A fixed point theorem for $L^{1}$ spaces. Invent. Math., 189(1):143-148, 2012.

[BH99] Martin R. Bridson and André Haefliger. Metric spaces of non-positive curvature, volume 319 of Grundlehren der Mathematischen Wissenschaften [Fundamental Principles of Mathematical Sciences]. Springer-Verlag, Berlin, 1999.

[BHS17a] Jason Behrstock, Mark F. Hagen, and Alessandro Sisto. Hierarchically hyperbolic spaces, I: Curve complexes for cubical groups. Geom. Topol., 21(3):17311804, 2017.

[BHS17b] Jason Behrstock, Mark F. Hagen, and Alessandro Sisto. Quasiflats in hierarchically hyperbolic spaces. arXiv:1704.04271v1, 2017.

[BHS19] Jason Behrstock, Mark Hagen, and Alessandro Sisto. Hierarchically hyperbolic spaces II: Combination theorems and the distance formula. Pacific J. Math., 299(2):257-338, 2019.

[BJ06] Armand Borel and Lizhen Ji. Compactifications of symmetric and locally symmetric spaces. Mathematics: Theory \& Applications. Birkhäuser Boston, Inc., Boston, MA, 2006.

[BJ07] Armand Borel and Lizhen Ji. Compactifications of symmetric spaces. J. Differential Geom., 75(1):1-56, 2007.

[BM00a] Marc Burger and Shahar Mozes. Groups acting on trees: from local to global structure. Inst. Hautes Études Sci. Publ. Math., (92):113-150 (2001), 2000.

[BM00b] Marc Burger and Shahar Mozes. Lattices in product of trees. Inst. Hautes Études Sci. Publ. Math., (92):151-194 (2001), 2000.

[BM02] Marc Burger and Nicolas Monod. Continuous bounded cohomology and applications to rigidity theory. Geom. Funct. Anal., 12(2):219-280, 2002.

[Bow13a] Brian H. Bowditch. Coarse median spaces and groups. Pacific J. Math., 261(1):53-93, 2013.

[Bow13b] Brian H. Bowditch. Invariance of coarse median spaces under relative hyperbolicity. Math. Proc. Cambridge Philos. Soc., 154(1):85-95, 2013.

[Bow16] Brian H. Bowditch. Some properties of median metric spaces. Groups Geom. Dyn., 10(1):279-317, 2016.

[Bow18] Brian H. Bowditch. Large-scale rigidity properties of the mapping class groups. Pacific J. Math., 293(1):1-73, 2018. 
[Cap09] Pierre-Emmanuel Caprace. Amenable groups and Hadamard spaces with a totally disconnected isometry group. Comment. Math. Helv., 84(2):437-455, 2009.

[CD17] Indira Chatterji and Cornelia Druţu. Median geometry for spaces with measured walls and for groups. arXiv:1708.00254v1, 2017.

[CDH10] Indira Chatterji, Cornelia Druţu, and Frédéric Haglund. Kazhdan and Haagerup properties from the median viewpoint. Adv. Math., 225(2):882-921, 2010.

[CdlH16] Yves Cornulier and Pierre de la Harpe. Metric geometry of locally compact groups, volume 25 of EMS Tracts in Mathematics. European Mathematical Society (EMS), Zürich, 2016. Winner of the 2016 EMS Monograph Award.

[CFI16] Indira Chatterji, Talia Fernós, and Alessandra Iozzi. The median class and superrigidity of actions on CAT(0) cube complexes. J. Topol., 9(2):349-400, 2016. With an appendix by Pierre-Emmanuel Caprace.

[CL10] Pierre-Emmanuel Caprace and Alexander Lytchak. At infinity of finitedimensional CAT(0) spaces. Math. Ann., 346(1):1-21, 2010.

[CM09] Pierre-Emmanuel Caprace and Nicolas Monod. Isometry groups of nonpositively curved spaces: structure theory. J. Topol., 2(4):661-700, 2009.

[CMV04] Pierre-Alain Cherix, Florian Martin, and Alain Valette. Spaces with measured walls, the Haagerup property and property (T). Ergodic Theory Dynam. Systems, 24(6):1895-1908, 2004.

[CN05] Indira Chatterji and Graham Niblo. From wall spaces to CAT(0) cube complexes. Internat. J. Algebra Comput., 15(5-6):875-885, 2005.

[Cor13] Yves Cornulier. Group actions with commensurated subsets, wallings and cubings. arXiv:1302.5982v2, 2013.

[CR09] Pierre-Emmanuel Caprace and Bertrand Rémy. Simplicity and superrigidity of twin building lattices. Invent. Math., 176(1):169-221, 2009.

[CR10] Pierre-Emmanuel Caprace and Bertrand Rémy. Non-distortion of twin building lattices. Geom. Dedicata, 147:397-408, 2010.

[CRK15] Montserrat Casals-Ruiz and Ilya Kazachkov. Limit groups over partially commutative groups and group actions on real cubings. Geom. Topol., 19(2):725852, 2015.

[CS11] Pierre-Emmanuel Caprace and Michah Sageev. Rank rigidity for CAT(0) cube complexes. Geom. Funct. Anal., 21(4):851-891, 2011.

[dCTV07] Yves de Cornulier, Romain Tessera, and Alain Valette. Isometric group actions on Hilbert spaces: growth of cocycles. Geom. Funct. Anal., 17(3):770-792, 2007.

[dCTV08] Yves de Cornulier, Romain Tessera, and Alain Valette. Isometric group actions on Banach spaces and representations vanishing at infinity. Transform. Groups, 13(1):125-147, 2008.

[Del77] Patrick Delorme. 1-cohomologie des représentations unitaires des groupes de Lie semi-simples et résolubles. Produits tensoriels continus de représentations. Bull. Soc. Math. France, 105(3):281-336, 1977.

[Dil50] Robert P. Dilworth. A decomposition theorem for partially ordered sets. Ann. of Math. (2), 51:161-166, 1950.

[DP16] Thomas Delzant and Pierre Py. Cubulable Kähler groups. arXiv:1609.08474v1, 2016.

[EO18] Anna Erschler and Narutaka Ozawa. Finite-dimensional representations constructed from random walks. Comment. Math. Helv., 93(3):555-586, 2018.

[Fer18] Talia Fernós. The Furstenberg-Poisson boundary and CAT(0) cube complexes. Ergodic Theory Dynam. Systems, 38(6):2180-2223, 2018.

[Fio17] Elia Fioravanti. Roller boundaries for median spaces and algebras. arXiv:1708.01005v2, 2017. 
[Fio18] Elia Fioravanti. The Tits alternative for finite rank median spaces. Enseign. Math., 64(1-2):89-126, 2018.

[FLM18] Talia Fernós, Jean Lécureux, and Frédéric Mathéus. Random walks and boundaries of CAT(0) cubical complexes. Comment. Math. Helv., 93(2):291333, 2018.

[FV17] Talia Fernós and Alain Valette. The Mayer-Vietoris sequence for graphs of groups, property $(\mathrm{T})$, and the first $\ell^{2}$-Betti number. Homology Homotopy Appl., 19(2):251-274, 2017.

[Gen19] Anthony Genevois. Hyperbolic and cubical rigidities of Thompson's group $V$. J. Group Theory, 22(2):313-345, 2019.

[Ger97] Victor N. Gerasimov. Semi-splittings of groups and actions on cubings. In $\mathrm{Al}$ gebra, geometry, analysis and mathematical physics (Russian) (Novosibirsk, 1996), pages 91-109, 190. Izdat. Ross. Akad. Nauk Sib. Otd. Inst. Mat., Novosibirsk, 1997.

[GH10] Erik Guentner and Nigel Higson. Weak amenability of CAT(0)-cubical groups. Geom. Dedicata, 148:137-156, 2010.

[Gro87] Mikhael Gromov. Hyperbolic groups. In Essays in group theory, volume 8 of Math. Sci. Res. Inst. Publ., pages 75-263. Springer, New York, 1987.

[Gro93] Mikhael Gromov. Asymptotic invariants of infinite groups. In Geometric group theory, Vol. 2 (Sussex, 1991), volume 182 of London Math. Soc. Lecture Note Ser., pages 1-295. Cambridge Univ. Press, Cambridge, 1993.

[Gui72] Alain Guichardet. Sur la cohomologie des groupes topologiques. II. Bull. Sci. Math. (2), 96:305-332, 1972.

[Gui05] Vincent Guirardel. Cœur et nombre d'intersection pour les actions de groupes sur les arbres. Ann. Sci. École Norm. Sup. (4), 38(6):847-888, 2005.

[Hae16a] Thomas Haettel. Higher rank lattices are not coarse median. Algebr. Geom. Topol., 16(5):2895-2910, 2016.

[Hae16b] Thomas Haettel. Hyperbolic rigidity of higher rank lattices. arXiv:160\%.02004v2, 2016.

[Hag07] Frédéric Haglund. Isometries of CAT(0) cube complexes are semi-simple. arXiv:0705.3386v1, 2007.

[Hag13] Mark F. Hagen. The simplicial boundary of a CAT(0) cube complex. Algebr. Geom. Topol., 13(3):1299-1367, 2013.

[Hag17] Mark F. Hagen. Corrigendum to "The simplicial boundary of a CAT(0) cube complex". 2017.

[HP98] Frédéric Haglund and Frédéric Paulin. Simplicité de groupes d'automorphismes d'espaces à courbure négative. In The Epstein birthday schrift, volume 1 of Geom. Topol. Monogr., pages 181-248. Geom. Topol. Publ., Coventry, 1998.

[Kat18] Motoko Kato. On groups whose actions on finite-dimensional CAT(0) spaces have global fixed points. arXiv:1804.10506v1, 2018.

[KK13] Marcin Kotowski and Michał Kotowski. Random groups and property $(T)$ : Żuk's theorem revisited. J. Lond. Math. Soc. (2), 88(2):396-416, 2013.

[Kle10] Bruce Kleiner. A new proof of Gromov's theorem on groups of polynomial growth. J. Amer. Math. Soc., 23(3):815-829, 2010.

[KS16] Aditi Kar and Michah Sageev. Ping pong on CAT(0) cube complexes. Comment. Math. Helv., 91(3):543-561, 2016.

[Lee00] Bernhard Leeb. A characterization of irreducible symmetric spaces and Euclidean buildings of higher rank by their asymptotic geometry, volume 326 of Bonner Mathematische Schriften [Bonn Mathematical Publications]. Universität Bonn, Mathematisches Institut, Bonn, 2000. 
[Mar91] Grigorii A. Margulis. Discrete subgroups of semisimple Lie groups, volume 17 of Ergebnisse der Mathematik und ihrer Grenzgebiete (3) [Results in Mathematics and Related Areas (3)|. Springer-Verlag, Berlin, 1991.

[Mar06] Florian Martin. Reduced 1-cohomology of connected locally compact groups and applications. J. Lie Theory, 16(2):311-328, 2006.

[Min16] Ashot Minasyan. New examples of groups acting on real trees. J. Topol., 9(1):192-214, 2016.

[Mon06] Nicolas Monod. Superrigidity for irreducible lattices and geometric splitting. J. Amer. Math. Soc., 19(4):781-814, 2006.

[Nic04] Bogdan Nica. Cubulating spaces with walls. Algebr. Geom. Topol., 4:297-309, 2004.

[Nic08] Bogdan Nica. Group actions on median spaces. arXiv:0809.4099v1, 2008.

[NS13] Amos Nevo and Michah Sageev. The Poisson boundary of CAT(0) cube complex groups. Groups Geom. Dyn., 7(3):653-695, 2013.

[NWZ18a] Graham A. Niblo, Nick Wright, and Jiawen Zhang. Coarse median algebras: The intrinsic geometry of coarse median spaces and their intervals. arXiv:1802.02499v2, 2018.

[NWZ18b] Graham A. Niblo, Nick Wright, and Jiawen Zhang. A four point characterisation for coarse median spaces. arXiv:1708.06960v2, 2018.

[Oll04] Yann Ollivier. Sharp phase transition theorems for hyperbolicity of random groups. Geom. Funct. Anal., 14(3):595-679, 2004.

[OW11] Yann Ollivier and Daniel T. Wise. Cubulating random groups at density less than 1/6. Trans. Amer. Math. Soc., 363(9):4701-4733, 2011.

[Oza18] Narutaka Ozawa. A functional analysis proof of Gromov's polynomial growth theorem. Ann. Sci. Éc. Norm. Supér. (4), 51(3):549-556, 2018.

[Pau88] Frédéric Paulin. Topologie de Gromov équivariante, structures hyperboliques et arbres réels. Invent. Math., 94(1):53-80, 1988.

[Pau91] Frédéric Paulin. Outer automorphisms of hyperbolic groups and small actions on R-trees. In Arboreal group theory (Berkeley, CA, 1988), volume 19 of Math. Sci. Res. Inst. Publ., pages 331-343. Springer, New York, 1991.

[Rém99] Bertrand Rémy. Construction de réseaux en théorie de Kac-Moody. $C . R$. Acad. Sci. Paris Sér. I Math., 329(6):475-478, 1999.

[Rém05] Bertrand Rémy. Integrability of induction cocycles for Kac-Moody groups. Math. Ann., 333(1):29-43, 2005.

[Rol98] Martin A. Roller. Poc sets, median algebras and group actions. An extended study of Dunwoody's construction and Sageev's theorem. Preprint, University of Southampton, 1998.

[Sag95] Michah Sageev. Ends of group pairs and non-positively curved cube complexes. Proc. London Math. Soc. (3), 71(3):585-617, 1995.

[Sha00] Yehuda Shalom. Rigidity of commensurators and irreducible lattices. Invent. Math., 141(1):1-54, 2000.

[Sha04] Yehuda Shalom. Harmonic analysis, cohomology, and the large-scale geometry of amenable groups. Acta Math., 192(2):119-185, 2004.

[ŠW17] Ján Špakula and Nick Wright. Coarse medians and property A. Algebr. Geom. Topol., 17(4):2481-2498, 2017.

[Ż03] Andrzey Żuk. Property (T) and Kazhdan constants for discrete groups. Geom. Funct. Anal., 13(3):643-670, 2003.

[Zei16] Rudolf Zeidler. Coarse median structures and homomorphisms from Kazhdan groups. Geom. Dedicata, 180:49-68, 2016. 